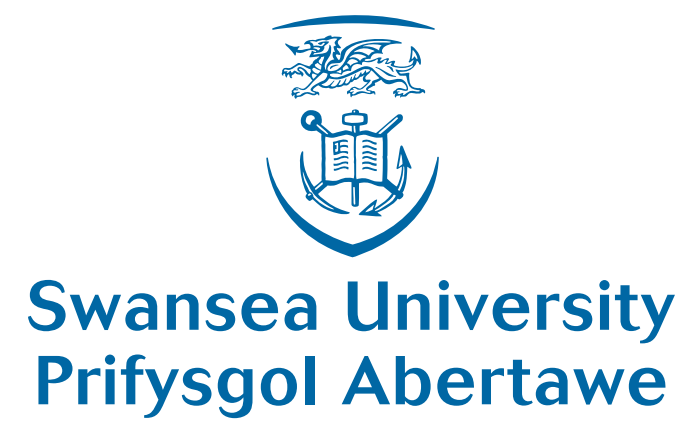

\title{
Dynamics of Three-Dimensional Pipes Conveying Fluid using the Reissner Beam Theory
}

\author{
by \\ Anthony Edwin MUOKA \\ Submitted to Swansea University \\ in fulfilment of the requirements for the Degree of \\ Doctor of Philosophy
}

2018 


\section{DECLARATION}

This work has not previously been accepted in substance for any degree and is not being concurrently submitted in candidature for any degree

Signed

Date

\section{STATEMENT 1}

This thesis is the result of my own investigations, except where otherwise stated. When correction services have been used, the extent and nature of the correction is clearly marked in a footnote(s).

Other sources are acknowledged by footnotes giving explicit references. A bibliography is appended.

Signed

Date.

\section{STATEMENT 2}

I herby give consent for my thesis, if accepted, to be available for photocopying and for inter-library loan and for the title and summary to be made available to outside organisations.

Signed

Date. 


\section{Acknowledgements}

My profound thanks goes to my supervisors, Prof. Djordje Perić and Dr. Wulf Dettmer without whom this work would not have been possible. Their patience, guidance and encouragement has been excellent throughout.

I would like to thank my parents and family for their constant support and continued encouragement through my studies, financially, spiritually, and in so many other ways. I am blessed to have you all in my life. Mummy, Daddy, may God continue to bless you.

To my colleagues and friends who have worked alongside me and been there for me, I say thank you very much. I am grateful for all your support and friendship during this period. Richard Evans, Mike Weberstadt, Dr. Bruce Jones, Phillippa Nwamu, Dumebi Umezinne, you guys are the best.

Finally to the members of the Catholic Society, Father Carlito, and Sister Bridget, you made a home for me away from home. I am grateful, and may God continue to bless you all.

Above all, to God be the glory, for his continued blessings upon me. 


\section{Summary}

The study of dynamics of pipes conveying fluid has been the subject of research for many decades now, and various formulations, solution methodologies and applications have been developed. The topic is well understood but research in this area is ongoing as the study of the subject is far from trivial. This is a classical model problem in the study of dynamics and stability of structures mainly because it is a physically simple system capable of displaying a wide array of interesting behaviour in both the linear and nonlinear regime. In this thesis, a geometrically exact fully implicit version of the $3 \mathrm{D}$ beam element, which employs the Rodrigues formula for the update of large rotations is used in the solution of the equations of motion. The nonlinear model for the flexible beam conveying fluid has been formulated and implemented to recover the interesting dynamic behaviour of the system in 3D. The advantage of this approach stems mainly from the fact that approach to engineering problems depends upon the intended application, cost from a computational perspective, among other factors which may be taken into consideration, and this provides an alternative to existing approaches. Benchmark problems are presented in $2 \mathrm{D}$ and $3 \mathrm{D}$, and confirm robustness and accuracy of the formulation. 


\section{Contents}

List of Figures viii

List of Tables $\quad$ xi

\begin{tabular}{lll}
\hline & Introduction & 1
\end{tabular}

1.1 Motivation . . . . . . . . . . . . . . . . . . . . 1

1.2 Aim of the Thesis . . . . . . . . . . . . . . . . . . . . 8

1.3 Layout of the thesis . . . . . . . . . . . . . . . . . . . . . . 8

$\begin{array}{lll}2 & \text { 3D Geometrically Exact Beam } & 10\end{array}$

$2.1 \quad$ Introduction . . . . . . . . . . . . . . . . . . . . . . . . . . . . . . . 10

2.2 Kinematics $\ldots \ldots \ldots \ldots \ldots \ldots$

2.3 Large Rotations . . . . . . . . . . . . . . . . . . . . . . . . . 15

2.3 .1 Rotation group . . . . . . . . . . . . . . . 15

2.3 .2 Parametrization of Finite Rotations . . . . . . . . . . . 16

2.3 .3 Configuration Space and Compound Rotations . . . . . . . . . 18

2.3 .4 Rotation parameters . . . . . . . . . . . . . . . . . . . . . 19

2.3 .5 Spatial derivative (Curvature) . . . . . . . . . . . 20

2.4 Strain measures . . . . . . . . . . . . . . . . . . . . . . . . . . . . . . 21

2.5 Equilibrium and virtual work equations . . . . . . . . . . . . . 23

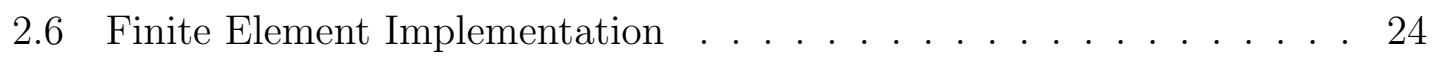

2.7 Numerical Results . . . . . . . . . . . . . . . . . . . . . . . . . . 26

2.7 .1 Small deflection test of a Cantilever Beam . . . . . . . . . . . 27

2.7.2 Large displacement and rotation test of a Cantilever Beam . . 27

2.7 .3 Roll-up of a Beam under end moment. . . . . . . . . . . . . . 30

2.7 .4 Buckling of a narrow cantilever Beam . . . . . . . . . . . . . 33

2.7 .5 Lee Frame . . . . . . . . . . . . . . . . . . . . . . . . . . . . . 34

2.7.6 Large displacement analysis of a $45^{\circ}$ Cantilever beam bend . . 36

2.8 Conclusion . . . . . . . . . . . . . . . . . . . . . . . 37 
$\begin{array}{lll}3 & \text { Vibration of beams } & 39\end{array}$

3.1 Introduction . . . . . . . . . . . . . . . . . . . . . . . . 39

3.2 Equation of motion . . . . . . . . . . . . . . . . . . . . . 40

3.3 Finite Element Implementation . . . . . . . . . . . . . . . . . . . . . 41

3.4 Time stepping scheme . . . . . . . . . . . . . . . . . . . . . . . . . . 43

3.5 Numerical example . . . . . . . . . . . . . . . . . . . . . . . . . . . . 44

3.5 .1 Lee's frame . . . . . . . . . . . . . . . . . . . . . 44

3.6 Conclusion $\ldots \ldots \ldots \ldots$. . . . . . . . . . . . . . . . . . . . . . . . . 45

4 Pipes Conveying fluid: 2D Linear dynamics 47

4.1 Introduction . . . . . . . . . . . . . . . . . . . . . . 47

4.2 Equations of motion . . . . . . . . . . . . . . . . . . 47

4.3 Finite Element Solution . . . . . . . . . . . . . . . . . . . 50

4.4 Time stepping scheme . . . . . . . . . . . . . . . . . 54

4.5 Numerical examples . . . . . . . . . . . . . . . . . . . . . 55

4.5 .1 Natural frequency of vibration . . . . . . . . . . . . . . 55

$4.5 .2 \quad$ Stability of pipes with simply supported ends . . . . . . . . 59

4.5 .3 Stability of cantilever pipes . . . . . . . . . . . . . 59

4.6 Conclusion . . . . . . . . . . . . . . . . . . . . . . . . . . 62

5 Pipes Conveying fluid: 2D nonlinear solution $\quad 64$

5.1 Introduction . . . . . . . . . . . . . . . . . . . . 64

5.2 Variational formulation . . . . . . . . . . . . . . . . . . . . . 64

5.2 .1 Pipe stiffness term . . . . . . . . . . . . . . . 64

5.2 .2 Centrifugal force term $\ldots \ldots \ldots 6$

5.2 .3 Inertia term . . . . . . . . . . . . . . . . . . . 67

5.2 .4 Coriolis force term . . . . . . . . . . . . . . . . . 67

5.3 Finite element formulation . . . . . . . . . . . . . . . . . . . . . . . 67

5.3 .1 Pipe stiffness term . . . . . . . . . . . . . . 67

5.3 .2 Centrifugal force term . . . . . . . . . . . . 70

5.3 .3 Inertia term . . . . . . . . . . . . . . . . . . 70

5.3 .4 Coriolis force term . . . . . . . . . . . . . . . . 71

5.4 Numerical examples . . . . . . . . . . . . . . . . . . . . . 71

$5.4 .1 \quad$ Natural frequency of vibration . . . . . . . . . . . . . . . 72

5.4 .2 Subcritical fluid flow through the pipe . . . . . . . . . . . 74

5.4 .3 Instability of pipes . . . . . . . . . . . . . 76

5.5 Conclusion . . . . . . . . . . . . . . . . . . . . 80 
6 Pipes Conveying fluid: 3D nonlinear solution 81

6.1 Introduction . . . . . . . . . . . . . . . . . . . . . 81

6.2 Finite element approximation $\ldots \ldots \ldots \ldots 1$

$6.2 .1 \quad$ Pipe stiffness term . . . . . . . . . . . . . . . . . . . . 81

6.2 .2 Centrifugal force term $\ldots \ldots \ldots \ldots$. . . . . . . . . 82

6.2 .3 Coriolis force term . . . . . . . . . . . . . . . . 84

6.2 .4 Inertia term . . . . . . . . . . . . . . . . . . . 85

6.3 Numerical examples . . . . . . . . . . . . . . . . . . . . 86

$6.3 .1 \quad$ Instability of cantilever pipes $\ldots \ldots \ldots \ldots$. . . . . . 86

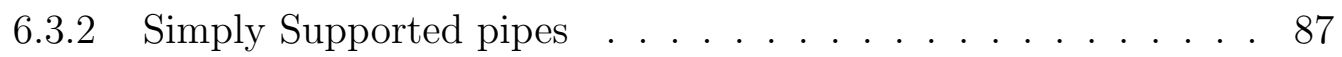

6.4 Conclusion . . . . . . . . . . . . . . . . . . . . . . . . . . . . 89

$\begin{array}{lll}7 \text { Conclusion } & 93\end{array}$

7.1 Suggestions for future research $\ldots \ldots \ldots \ldots$. . . . . . . . 94

\begin{tabular}{ll}
\hline Bibliography & 95
\end{tabular} 


\section{List of Figures}

$1.1 \quad$ Various Riser types in dynamic subsea environments . . . . . . . . . 2

$1.2 \quad$ Pipelines conveying petroleum products . . . . . . . . . . . . 2

$1.3 \quad$ Failure of pipelines and damage to the environment . . . . . . . . 3

1.4 Pollution of water bodies . . . . . . . . . . . . . . . . . . . . . 3

1.5 An example of utilization of pipes conveying fluid in car engines and laboratory equipment . . . . . . . . . . . . . . . . 4 4

$1.6 \quad$ A healthy jet engine, and a damaged engine after emergency landing 4

$\begin{array}{lll}1.7 & \text { Pipe systems used to convey fluid for irrigation purposes } \ldots . . . & 5\end{array}$

1.8 Concrete pump delivering non-newtonian fluid . . . . . . . . . . . 5

1.9 MRI Scan of the pulmonary system . . . . . . . . . . . . . 6

1.10 Simulation of blood flow through an arterial network $\ldots . . . . \quad 7$

1.11 An infusion pump and a blood purification device used for treatment 7

$2.1 \quad$ Kinematic assumption $\ldots \ldots \ldots \ldots \ldots$

$2.23 \mathrm{D}$ representation of the coordinate system, including the reference

and deformed configurations . . . . . . . . . . . . . . 13

$2.3 \quad$ Rotation tensor $\Lambda$. . . . . . . . . . . . . . . . . 14

2.42 node linear shape functions and interpolation. . . . . . . . . . . 26

2.5 Geometry . . . . . . . . . . . . . . . . . . . 27

2.7 Geometry $\ldots \ldots \ldots \ldots \ldots$. . . . . . . . . . . . . . . . . . 28

$2.6 \quad$ Deflection under applied load, $P$ at the free end . . . . . . . . . 28

2.9 Geometry $\ldots \ldots \ldots \ldots$. . . . . . . . . . . . . . . . . . . . . 29

$2.8 \quad$ Large Cantilever Beam configurations under applied end moment . 30

$2.10 \quad$ Large beam rotation (twist) under end torsion . . . . . . . . . . . . . . . . 31

2.11 Geometry . . . . . . . . . . . . . . . . . . . . . . . . . . . 32

2.12 Roll-up of a beam under end moment . . . . . . . . . . . . . . . 32

2.13 Geometry . . . . . . . . . . . . . . . . . . . 34

2.14 Buckling of a narrow Cantilever Beam. . . . . . . . . . . . . . . 34

2.15 Geometry . . . . . . . . . . . . . . . . . . 35 
2.16 Large beam displacement under end moment . . . . . . . . . . . . . 35

2.17 Deformed shape of the $45^{\circ}$ Cantilever bend . . . . . . . . . . . . 36

2.18 Large displacement analysis of a $45^{\circ}$ Cantilever beam bend . . . . . 38

3.1 2 node linear shape functions . . . . . . . . . . . . . . . . . . . . 42

3.2 Geometry . . . . . . . . . . . . . . . . . . . 44

3.3 horizontal displacement . . . . . . . . . . . . . . . . . 45

$3.4 \quad$ vertical displacement . . . . . . . . . . . . . . . . . . 46

$4.1 \quad$ Pipe conveying fluid at constant velocity $v$. . . . . . . . . . . 48

$4.2 \quad$ Forces acting on the fluid element . . . . . . . . . . . . . . . . . 49

$4.3 \quad$ Forces acting on the solid element . . . . . . . . . . . . . . . . . . . 49

4.4 Hermitian shape functions . . . . . . . . . . . . . . . . . 51

4.5 Geometry . . . . . . . . . . . . . . . . 56

$4.6 \quad$ Undeformed configuration of the pipe . . . . . . . . . . . 56

4.7 Deformed configuration of the pipe . . . . . . . . . . . . . 57

4.8 Displacement history of the mid-span of a simply supported pipe undergoing free vibration . . . . . . . . . . . . . 57

4.9 Geometry . . . . . . . . . . . . . . . . . . 57

4.10 Deformed configuration of the pipe . . . . . . . . . . . . . 58

4.11 Displacement history of the free end of a cantilever pipe undergoing free vibration . . . . . . . . . . . . . . . . . . . . . . . . . 58

4.12 Geometry . . . . . . . . . . . . . . . . . . . 59

4.13 Frequency reduction with increasing flow velocity . . . . . . . . . 60

4.14 Displacement history of the mid-span of a simply supported pipe for various flow velocities . . . . . . . . . . . . . . . . . . 60

4.15 Displacement history of the mid-span of a simply supported pipe for various flow velocities . . . . . . . . . . . . . . . 60

4.16 Geometry . . . . . . . . . . . . . . . . . . . . . 61

4.17 Displacement history of the free end of a cantilever pipe for various flow velocities . . . . . . . . . . . . . . . . . . 61

4.18 Deformed cantilever pipes conveying fluid at velocities around the critical velocity . . . . . . . . . . . . . . . . . . . . . . . . . 62

$5.1 \quad$ Beam Kinematics $\ldots \ldots \ldots$. . . . . . . . . . . . . . . . . . . . 65

$5.2 \quad 2$ node linear shape functions and interpolation . . . . . . . . . . 68

5.3 Geometry . . . . . . . . . . . . . . . . . 72

5.5 Geometry . . . . . . . . . . . . . . . . . 72 
5.4 Displacement history of the mid-span of a simply supported pipe undergoing free vibration . . . . . . . . . . . . . . . . 73

$5.6 \quad$ Displacement history of the free end of a cantilever pipe undergoing free vibration . . . . . . . . . . . . . . . . 73

5.7 Geometry . . . . . . . . . . . . . . . . . . 74

5.8 Displacement history of the mid-span of a simply supported pipe for various flow velocities . . . . . . . . . . . . . . . . . . 74

$5.9 \quad$ Current configuration for the simply supported pipe $(v=7)$. . . 75

5.10 Geometry . . . . . . . . . . . . . . . . . . 75

5.11 Displacement history of the free end of a cantilever pipe for various flow velocities . . . . . . . . . . . . . . 76

5.13 Displacement history of the free end of a cantilever pipe for velocities beyond the critical velocity . . . . . . . . . . . . . . . . 77

5.12 Geometry . . . . . . . . . . . . . . . . . 77

$5.14 v=16.9 \ldots \ldots \ldots \ldots \ldots \ldots$

5.15 Pipe configuration for flow with velocities beyond the critical velocity 78

5.16 Geometry . . . . . . . . . . . . . . . . . 78

5.17 Buckling instability of a simply supported pipe with fluid flowing at a velocity above the critical velocity . . . . . . . . . . . . 79

5.18 Instability of a simply supported pipe . . . . . . . . . . . . . . 79

$6.1 \quad$ Initial and deformed cantilever pipe $v=9.2 \ldots \ldots \ldots$. . . . . . 87

6.2 Comparison of free-end displacement history for $v=9.2$, for $2 \mathrm{D}$ and

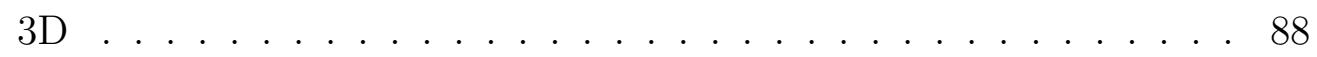

$6.3 \quad$ Initial and deformed cantilever pipe $v=20 \ldots \ldots$. . . . . . . . 88

$6.4 \quad$ Comparison of free-end displacement history for $v=20$. . . . . . . 89

6.5 Chaotic oscillations . . . . . . . . . . . . . . . . . 90

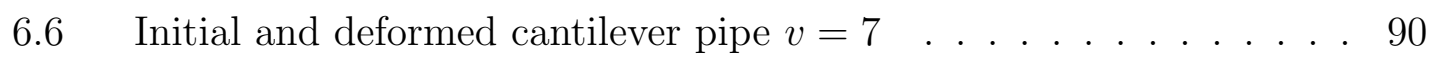

$6.7 \quad$ Comparison of free-end displacement history for $v=7$. . . . . . . 91

$6.8 \quad$ Initial and deformed cantilever pipe $v=12 \ldots \ldots$. . . . . . . 91

6.9 Comparison of free-end displacement history for $v=12$. . . . . . 91 


\section{List of Tables}

$2.1 \quad$ Comparison of results for the small deflection case . . . . . . . . . . 27

$2.2 \quad$ Convergence table for 10 load steps . . . . . . . . . . . . . . . 29

$2.3 \quad$ Comparison of results for the large deflection case . . . . . . . . . . 29

2.4 Convergence table for the 4,20 and 50 load step cases . . . . . . . . 33

$2.5 \quad$ Convergence table for 100 load steps . . . . . . . . . . . . 36

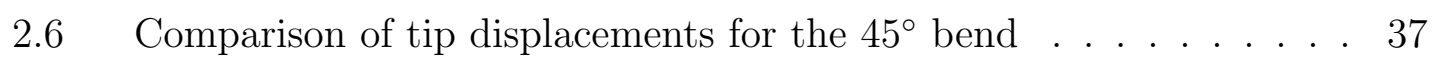

$2.7 \quad$ Convergence table for 10 load steps $\ldots \ldots$. . . . . . . . . . . . 37

3.1 Table of residuals for the last 2 time steps of the simulation . . . . . 45

5.1 Convergence table for 2 arbitrarily chosen time steps for velocity, $7 m s^{-1} \ldots \ldots \ldots \ldots \ldots \ldots \ldots$

5.2 Convergence table for 2 arbitrarily chosen time steps for velocity, $9.2 m s^{-1} \ldots \ldots \ldots \ldots \ldots \ldots$

5.3 Convergence table for 2 arbitrarily chosen time steps for velocity, $20 m s^{-1} \ldots \ldots \ldots \ldots \ldots 77$

5.4 Convergence table for 2 arbitrarily chosen time steps for velocity, $12 m s^{-1} \ldots \ldots \ldots \ldots \ldots \ldots$

$6.1 \quad$ Table of residuals for $\mathrm{v}=20$. . . . . . . . . . . . . . . . . . . 87

$6.2 \quad$ Table of residuals for $\mathrm{v}=12$. . . . . . . . . . . . . . . . . . . . . 89 


\section{Chapter 1}

\section{Introduction}

\subsection{Motivation}

The study of the dynamics of pipes conveying fluid is a very important one in Computational Multiphysics. A significant number of work has been done in this area and researchers are doing more work. Described by Paidoussis [45] as curiosity driven rather than with focus on possible applications, much of the work has led to the development of new mathematical techniques, as well as unforeseen applications to practical problems in various fields.

The rich rewards of the study of this class of problems can be seen in problems with some sort of flexible thin structure conveying fluid including but not limited to the nuclear industry, aeronautics and space field, and petrochemical engineering.

In the oil and gas industry, which is the main motivation for this work, we encounter pipes conveying fluid in many forms and applications, primarily risers and oil pipelines. Risers are simply conduits that serve as the main method for transporting hydrocarbons from the ocean floor to the host facility. These can also be used as a means to inject chemicals and water, for production purposes. The subsea oil and gas exploration environment consists of a dynamic one, and risers have various types and applications. Figure 1.1 shows typical uses of these risers, and highlights some of the different types.

Attached and pull tube risers, considered as one of the simpler designs are mainly utilized on fixed platforms, compliant towers, and are limited to water depths where the platforms can be deployed. Catenary risers, usually free hanging, quickly get complex as deeper waters are looked into, and are applicable to a wide number of designs. Flexible risers are specially made to demonstrate excellent bending capabilities, while possessing exceptional strength, making it extremely versatile for use in various situations. These usually undergo very large deformations to which this work 


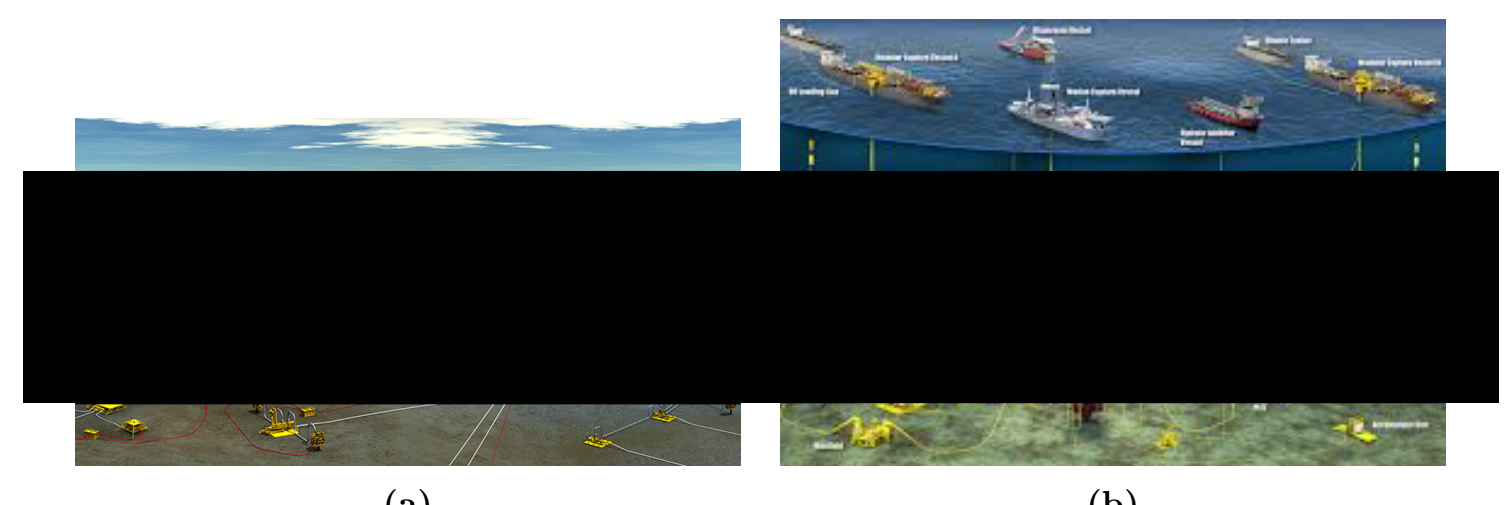

(a)

(b)

Figure 1.1: Various Riser types in dynamic subsea environments: (a) from www.genesisoilsndgas.com, and (b) from www.openpr.com (Global Oil and Gas Subsea Umbilicals, Risers and flowlines)

could be beneficial in the analysis and design of the systems. Top tensioned and hybrid risers are some others which are used depending on the situation. The process of oil and gas recovery is continually evolving, requiring more advanced technology, and consideration of additional factors in the design of the various systems.

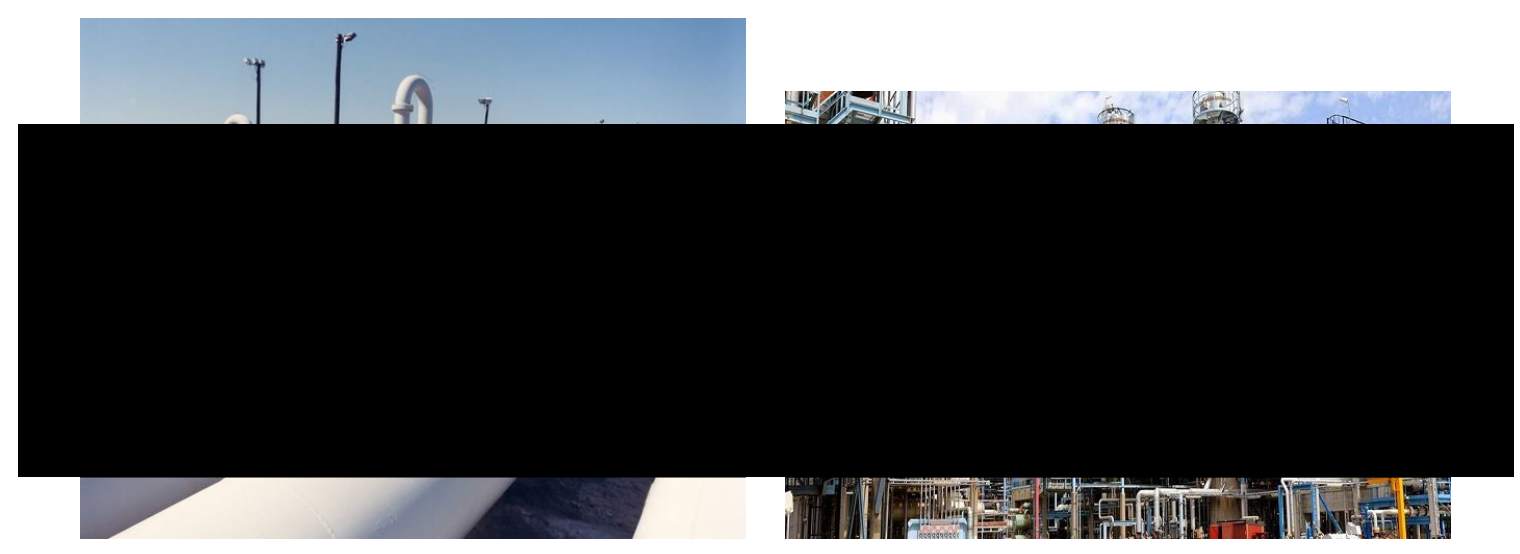

(a)

(b)

Figure 1.2: Pipelines conveying petroleum products: (a) from skymapglobal.com (Remote Sensing Oil and Gas Pipeline monitoring, and (b) from www.360nobs.com (Katsina Oil Refinery)

Oil pipelines are the primary means by which petroleum products are moved over very long distances. Damage to these pipes, or their failure, hence spillage and pollution, has serious effects on the environment, and the safety of the populace. Some of these systems and arrangements are shown in Figure 1.2, while Figures 1.3, and 1.4 shows potential devastation failure and damage of pipes and risers respectively can have on the environment.

As is the case with most engineering structures, huge deformation is generally undesirable, unless that is the intended purpose of the structure, and the structures are designed accordingly. Some of the other potential applications however do, and 


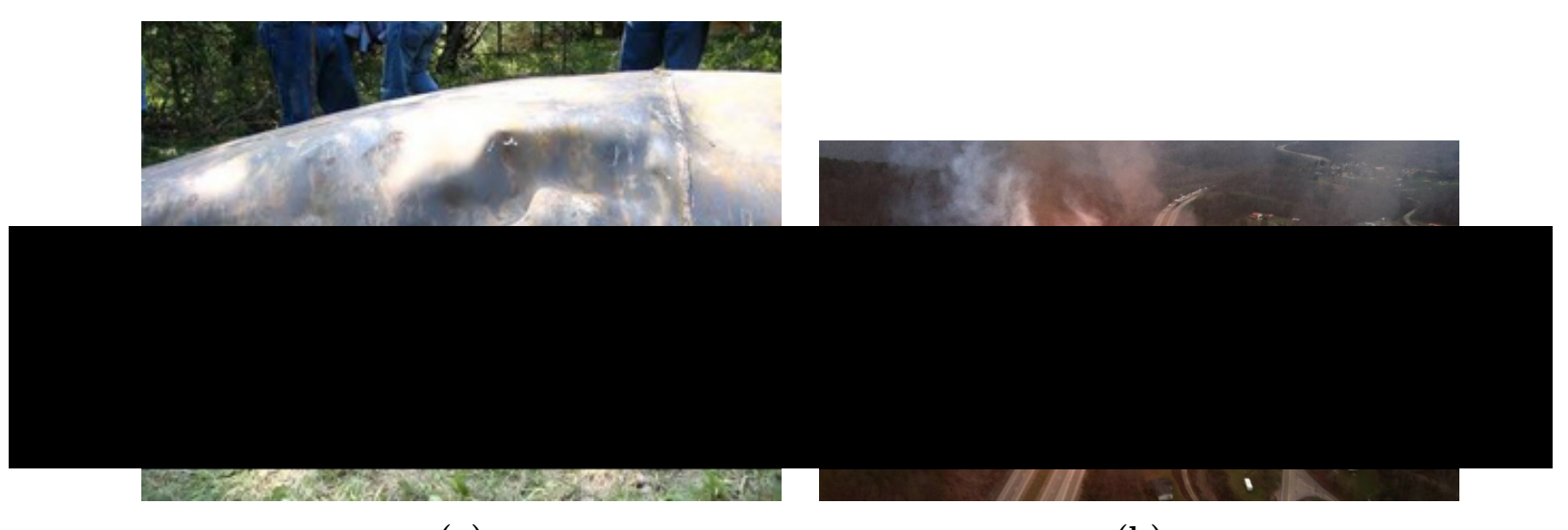

(a)

(b)

Figure 1.3: Failure of pipelines and damage to the environment: (a) from spectrabusters.org (Pilot Grove MO PEPL explosion), and (b) from www.nationofchange.org (Transcanada pipeline explodes)

sometimes these structures experience large deformations regardless, and a linear (small deformation) analysis will not be sufficient. In this work we have implemented the full nonlinear elements capable of analyzing the behaviour pipes conveying the fluid. Starting with the quasi-static analysis in 2D and 3D, moving to linear and nonlinear dynamics, and adding on the ability to simulate deformation of pipes conveying fluids, the formulation is capable of capturing the behaviour of most of these systems.

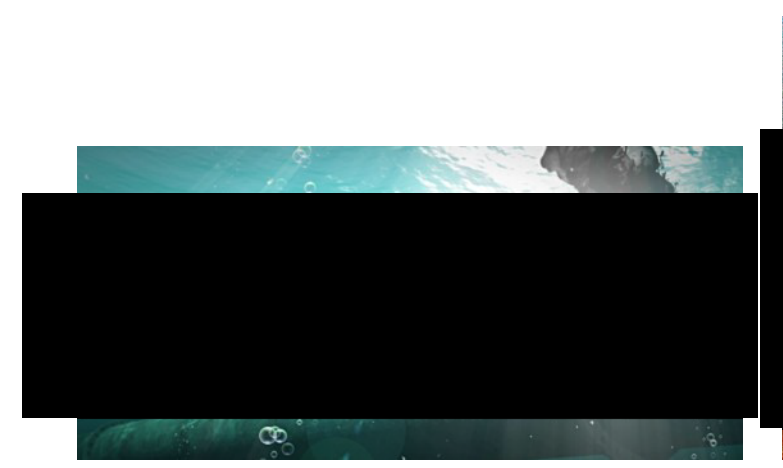

(a)

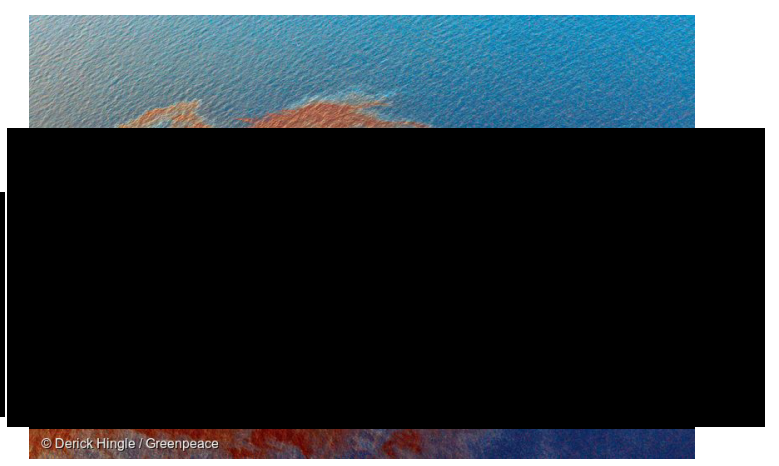

(b)

Figure 1.4: Pollution of water bodies: (a) from chesssubseaengineering.com (Offshore Pipeline failure), and (b) from www.greenpeace.org (90000 gallon Oil spill)

Another very good example of application of flow through pipes is in machinery. Vehicle engines, jet engines, laboratory equipment, and cooling systems of nuclear reactors are but a few, and these contain numerous pipes constantly conveying fluid at high velocities (Figure 1.5). Failure of these systems can often be catastrophic, leading to loss of lives, so care must be taken in analysis, design, and manufacture of the various components. The interaction between the solid pipe, and the fluid flowing within needs to be well understood, to ensure long term stability of the equipment, 
or scheduled maintenance and replacements, perhaps due to other factors such as fatigue or corrosion.

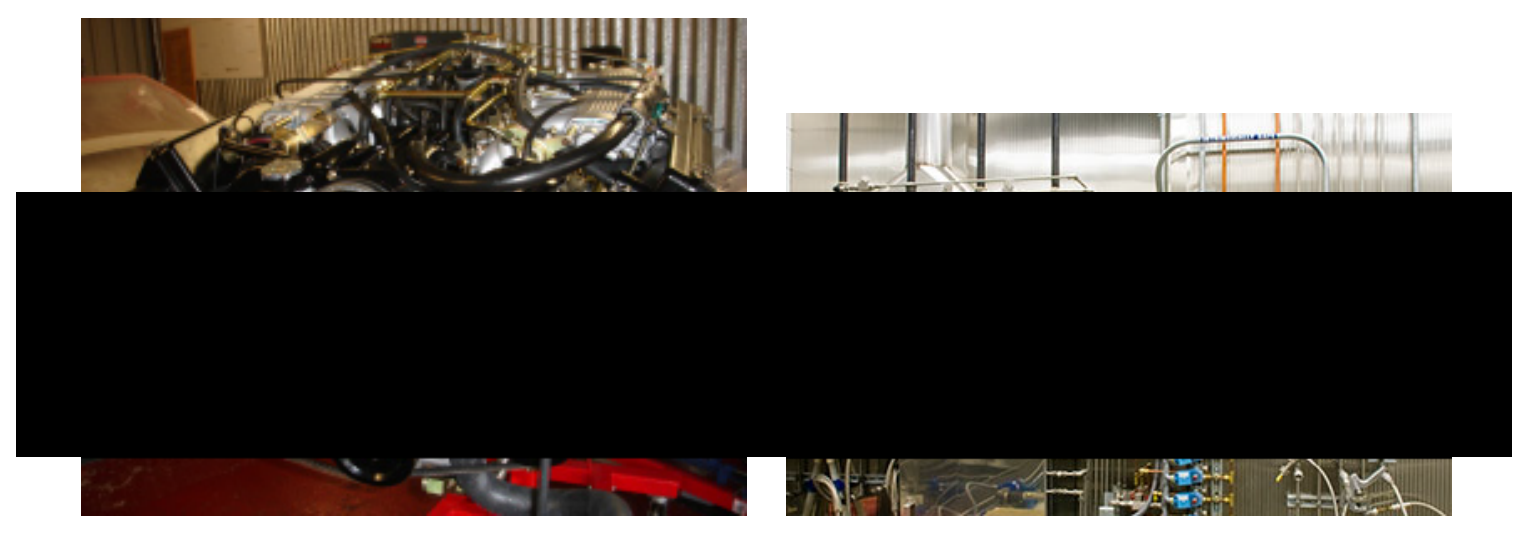

(a)

(b)

Figure 1.5: An example of utilization of pipes conveying fluid in car engines and laboratory equipment: (a) from www.rushtonengineering.co.uk (Classic Engines), and (b) from www.rmhgroup.com (Process Piping)

A mid flight engine explosion on a jumbo jet which forced an emergency landing in 2010, was reported to be as a result of an oil pipe failure. The failure of the pipe caused oil to leak into the engine, leading to a fire, and prompting a disastrous domino effect. This was a serious and rare event leading to tens of millions in compensation. The damaged engine is shown in Figure 1.6.

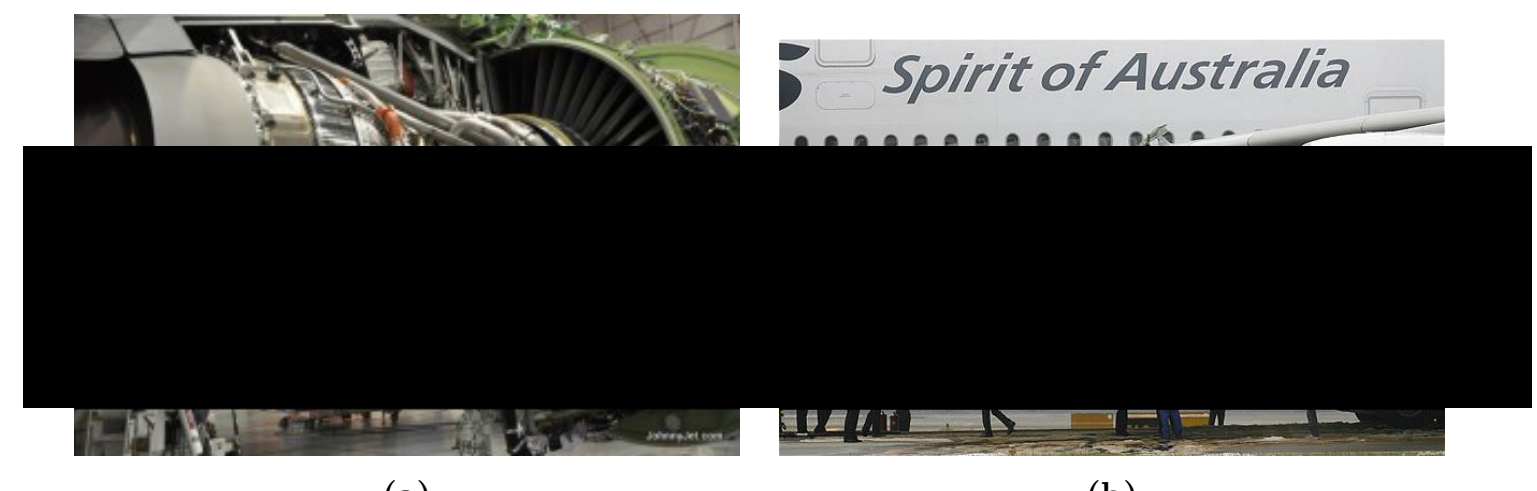

(a)

(b)

Figure 1.6: A healthy jet engine (a) and a damaged engine after the emergency landing (b): (a) from www.johnnyjet.com (Boeing Factory tour), and (b) from www.telegraph.co.uk (A380 Engine explosion)

The well known garden hose instability phenomenon, where the pipe flails about under high flow through it, is another good example. In plumbing, irrigation, and farming, to name a few, these pipes need to be chosen to withstand the expected pressures and velocities, to avoid damage and incurring costs. Wastewater and stormwater pipe systems are typical Civil engineering examples which fall into this category (Figure 1.7), concrete mixers delivering non-newtonian fluids for use on 
construction sites as well (Figure 1.8). Aspirating pipes, and pipes on elastic foundations also need to be considered, as some of the interesting dynamic behaviour obtained from simulations have been motivated by these systems. The rumbling of household plumbing can be attributed to water hammer which is a deflection of the pipe due to accelerating fluid, valve chatter, and pipe whip are also possible dynamic responses of pipes.

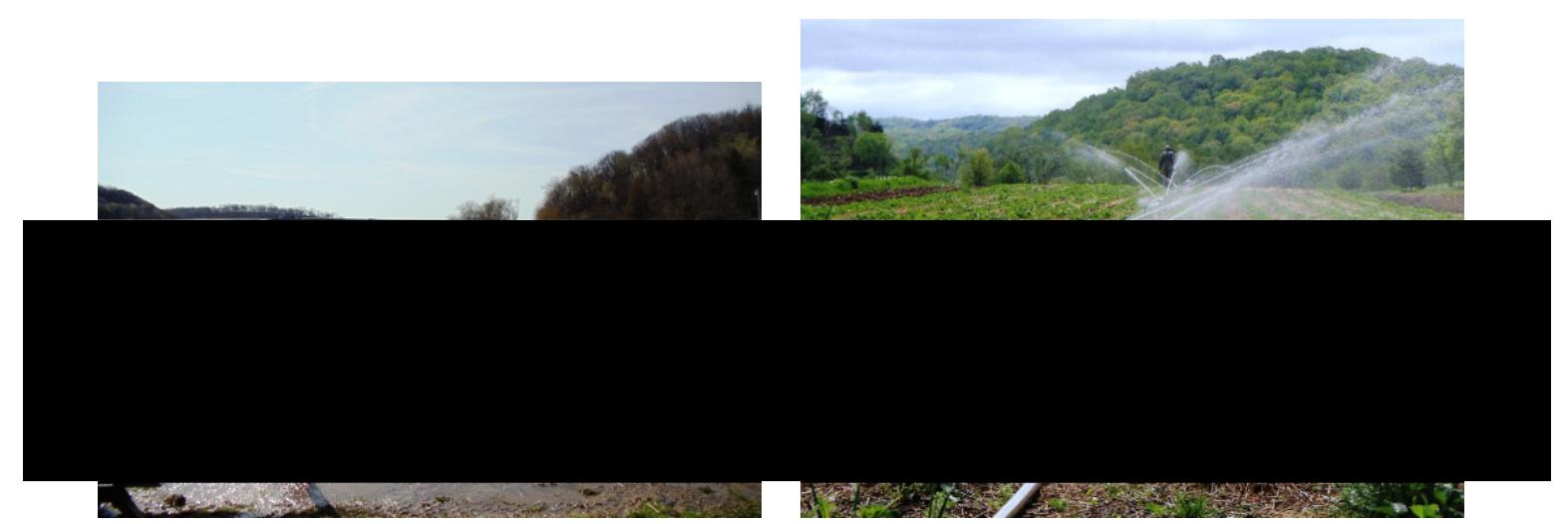

Figure 1.7: Pipe systems used to convey fluid for irrigation purposes. Images from vermontvalleyfarm.wordpress.com

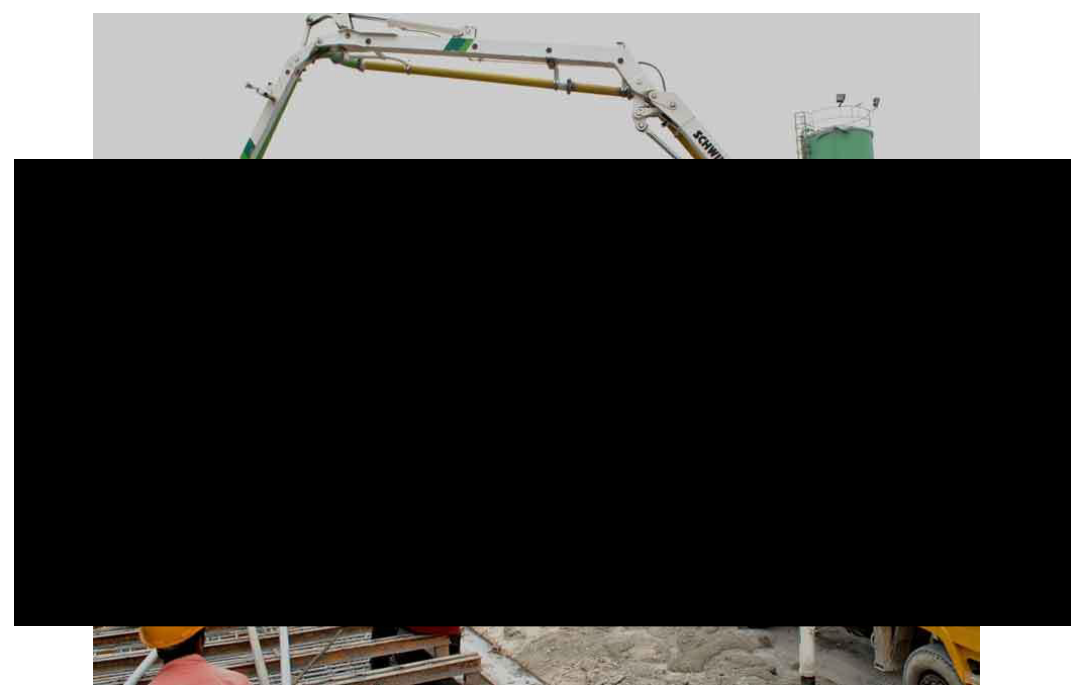

Figure 1.8: Concrete pump delivering non-newtonian fluid. Image from solusikonstruksi.com

In Biomedical engineering for example, engineering principles and techniques are applied to biology and medicine. Biomedical engineers work to develop new technologies, further research and continually push the boundaries of what is medically possible. This could be through establishing safety standards for devices, developing diagnostic tools, or furthering the understanding of the physics of various problems. One excellent example is the blood flow in human arterial system which is a fluid dynamics problem. Simulation of blood flow in the arterial network system will lead 
to a better understanding of the physiology of human body, assist with diagnosis and treatment of diseases associated with the cardiovascular system.

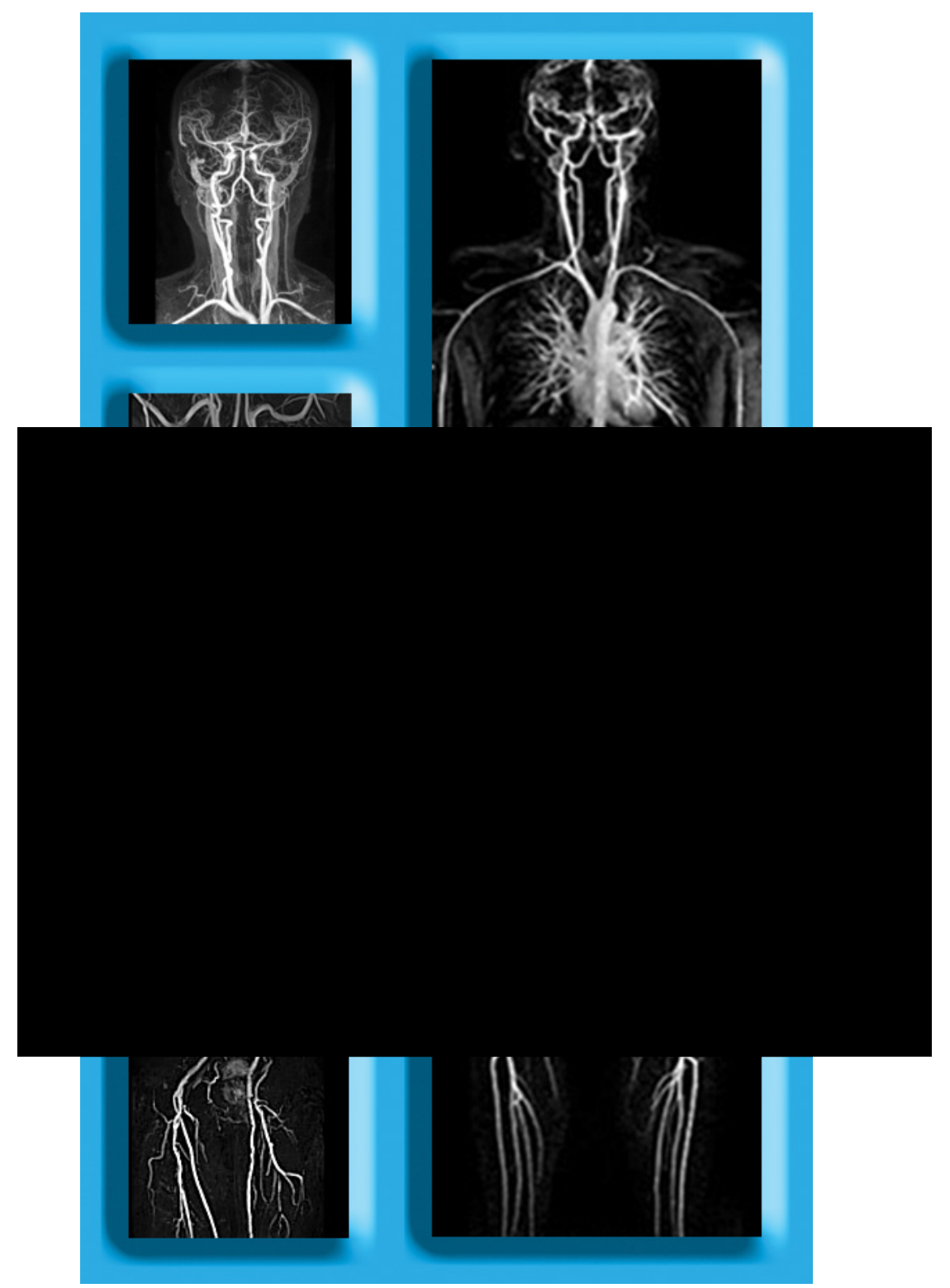

Figure 1.9: MRI Scan of the pulmonary system. Image from www.hitachimed.com

The flow of blood in the circulatory system is essentially a very complicated system of pipes conveying fluid. Figure 1.9 shows the system, and although the system is extremely complicated, comprising relatively large arteries and veins branching out into many, much smaller capillaries throughout the body, engineering principles can and have been used to study the interaction (see eg. Figure 1.10).

Medical devices on the other hand such as the infusion pump, used to deliver fluids into a patients body in a controlled manner, or devices for treatment, such as those used in blood purification and dialysis utilize pipes conveying fluids in various 


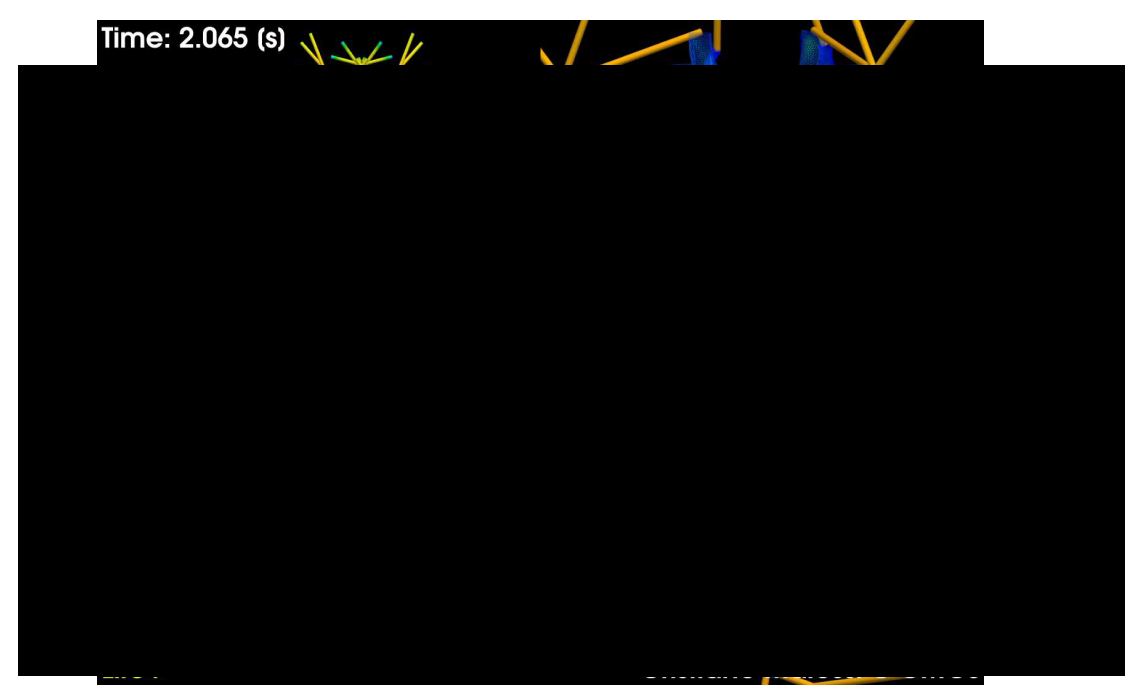

Figure 1.10: Simulation of blood flow through an arterial network. Image from www.youtube.com (Numerical Simulation of a Systemic Arterial Network)

forms. Be it for fluid extraction, delivery or monitoring purposes, these devices have to be designed properly and continually monitored to ensure the safety of patients.

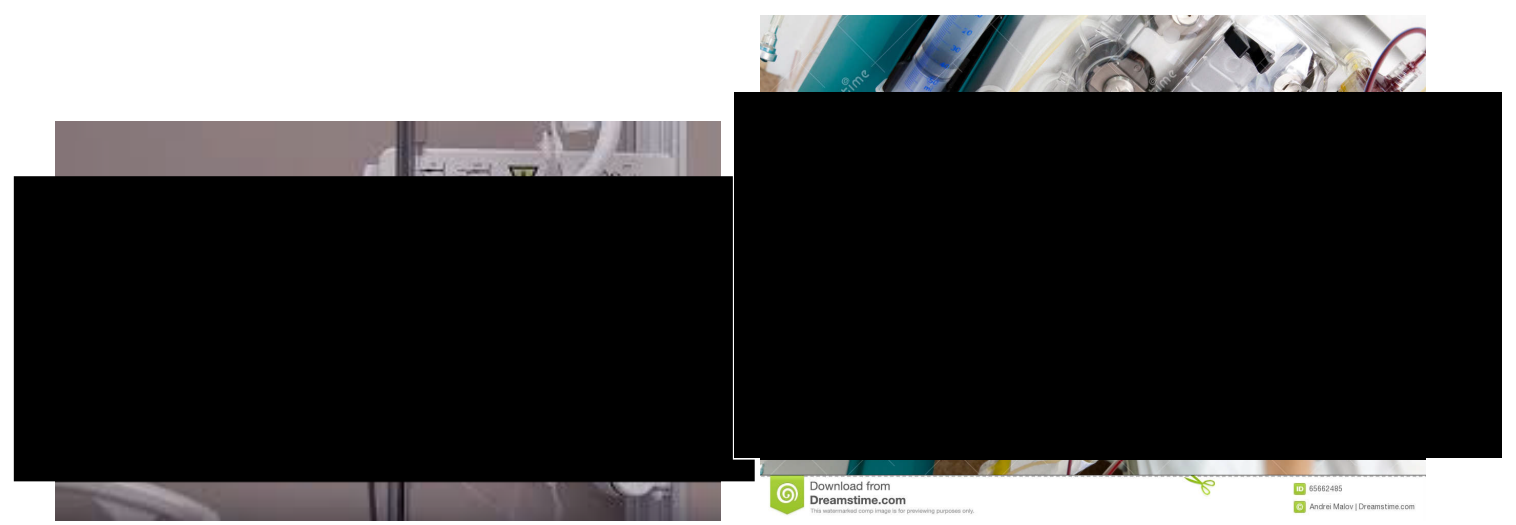

Figure 1.11: An infusion pump and a blood purification device used for treatment. Images (a) from www.coherentnews.com, and (b) from www.dreamstime.com

While in some of these examples failure is not necessarily a result of excessive vibrations of the various pipes, understanding the dynamic behaviour is very important as this could lead to failure of the system due to some other cause, probably accelerated by vibrations. Some of the research in this field have been a result of investigations into causes of accidents, damages, and even general discomfort due to acoustic considerations, to which the nonlinear dynamic response of these systems were a factor.

Some of the methods that have been proposed to study this class of problems include the Finite element method[58], differential quadrature method, transfer matrix method, dynamic stiffness method, Galerkin method, multiple scales method and recently, a new method based on Laplace transform [60]. 
Systems with varying additional supports, conical pipes, aspirating pipes, pipes on elastic foundations, to name a few are some additional problems that display interesting dynamic behaviour which is a defining quality of the study of this class of problems. Divergence and flutter are some of the examples of behaviours of pipes associated with instability, chaotic oscillations are also interesting results to obtain. This is indeed a model dynamic problem which will continue to attract work in the future.

\subsection{Aim of the Thesis}

The aim of the thesis is to formulate and implement a model for a flexible beam structure conveying fluid. Primarily implementing a geometrically nonlinear version of 3D beam element including an update of large rotations based on the Rodrigues formula, subjected to additional forces from the conveyed fluid. The goal being to model practical problems studied in literature and possibly chaotic oscillations as well with the code that will be developed. The attention here is restricted to incompressible fluids conveyed and negligible pipe elongation (inextensible).

The solution strategy to be implemented will be based on the finite element formulation for the elements. The finite element software; MPAP2 (Multi Physics Analysis Program) developed and maintained by Dr. Wulf G. Dettmer in Swansea University will be used, to which the code to be developed will be added as separate subroutines.

\subsection{Layout of the thesis}

This thesis is divided into 6 additional chapters.

In chapter 2, the geometrically exact $3 \mathrm{D}$ beam is presented. A treatment of large rotations and the Rodrigues formula is included with the governing equations. Benchmark problems are considered to show the accuracy of the method.

Chapter 3 provides a brief review of the literature on Dynamics of beams, and includes a couple of examples to show off the 2D and 3D element's ability to solve dynamic problems.

Chapter 4 begins our focus on dynamics of pipes conveying fluid. Here we present a linear solution of the governing equations of motion, include the time stepping scheme used and consider a few numerical examples to validate the results.

In chapter 5, we solve the equations of motion but for problems with large displacements and rotations and able to capture structural response beyond critical 
velocities. We observe some of the interesting dynamic behaviours of these systems here.

Chapter 6 presents the 3D model for the pipe conveying fluid. The governing equations are presented followed with numerical examples of relevant problems compared against results available in literature.

Chapter 7 brings the thesis to a close with conclusions of the work and recommendations for further work. 


\section{Chapter 2}

\section{D Geometrically Exact Beam}

This chapter of the work includes the 3D geometrically exact beam element, and benchmark examples to illustrate its performance. The kinematics, proper treatment of large rotations, governing equations, linearization and solution are presented, and the numerical examples chosen test various types of deformation of the beam in 3D.

\subsection{Introduction}

Since Euler [23] a one-dimensional continuum referred to as a beam is used as an adequate representation for the class of three-dimensional bodies having two of the three dimensions significantly smaller than the third. These are very important structural elements in the engineering practice, for example in the fields of Civil and Mechanical engineering, with structure such as frames, masts, car bodies, offshore pipes and risers, domes, robots, and general machines. A reliable mathematical and mechanical description of beams is of great significance and has attracted considerable interest in recent years.

Most of the formulations for the three-dimensional beam element are based on either a classical total Lagrangian formulation, or a corotational formulation, with many applications and variations of both. The classical formulation, which this work is based on, deals with the use of displacement and rotation with respect to a fixed inertial frame to describe the motion. Reissner's work [49], [50], [51] on the formulation of one-dimensional large strain beam theory for deformation of plane beams, and research on curved beams as well, is well known and has been built upon for many years. Ibrahimbegovic [28], and Makinen [42] are some of such researchers, implementing elements whose reference axis are arbitrary space-curved lines, and employ elements based on the Total Lagrangian Updating procedure, with rotation vector as dependent variable and dealing with the singularity issue for 
problems with greater than $2 \pi$ rotation. Additional work on finite rotations include [5], [12], 31], [53], 34]. Taking into account torsion and accompanying effects are the works of Jelenic[37], and Petrov [46] with applications to curved and twisted beams. Gruttman [25] looked into space curved beams with arbitrary cross sections, readily applicable to shells, and Auricchio[4] built upon Simo's work to derive model equations for general finite deformation cases in a consistent way.

The corotational formulation on the other hand, while also being a Total Lagrangian Formulation, approaches the problem differently. Primarily decomposing the motion into a rigid body motion with a local coordinate system fixed to the element, which rotates and moves with it. The other part is the deformation, which is measured in the local system. Adopted by so many researchers, this is also a very popular approach which has received much attention as well. This framework has been adopted by several researchers in the quest for more accurate and robust nonlinear beam and shell elements. Including work on the subject matter in the following works, [44] [22] [47] [19], Battini [7] formulated co-rotational element for buckling and post-buckling analysis of frames, investigating parametrization of finite rotations, and looking into the issue concerning warping effects. Hsia[26] investigated the nonlinear coupling among bending twisting and stretching deformations in the formulation. Vectorial rotational variables have also been used to implement a beam element and accurately model frame structures with large displacements and rotations [41].

Another crucial aspect of our review of existing literature is finite rotations. Three dimensional beam elements are not just simple extensions of two dimensional ones. Special treatment is required as is careful consideration before a choice of parametrization is made. Detailed information on the topic can be found in [1] [3] [20] [31] [29] [59] to name a few.

\subsection{Kinematics}

Based on the Reissner-Simo Beam theory used here, the beam is fully described by the position of its centroid and the orientation of its cross-sections. The centroids of these cross-sections are connected by a curve which is referred to as the line of centroids. For the sake of simplicity, let us assume that the line of centroids of the beam in the reference configuration is a straight line which coincides with the X-axis of the Cartesian coordinate system with $\boldsymbol{E}_{1}, \boldsymbol{E}_{2}$ and $\boldsymbol{E}_{3}$ as unit base vectors. The cross-sections of the beam are orthogonal to the line of centroids, and their normals lie along the base vector $\boldsymbol{E}_{1}$. The principal axes of inertia of the cross-sections are 
also taken to lie along the second and third base vectors $\boldsymbol{E}_{2}$ and $\boldsymbol{E}_{3}$, forming an orthonormal triad.

In the initial/reference configuration, the position vector of a particle on the line of centroids is denoted by $\boldsymbol{X}_{0}\left(\boldsymbol{X}_{1}\right) ; \boldsymbol{X}_{1} \in[0, L] \subset \mathbb{R}$, where $L$ is the beam length.

Introducing the basic kinematic assumptions here which doesn't affect the reproduction of large displacements and rotations, we have that:

(i) as a result of shearing of the beam, in the current/deformed configuration, the cross-section does not necessarily remain normal to the line of centroids (see Figure 2.1].

(ii) the Bernoulli hypothesis stating that the plane cross-sections remain plane after the deformation process, maintaining its area and shape is assumed to hold.

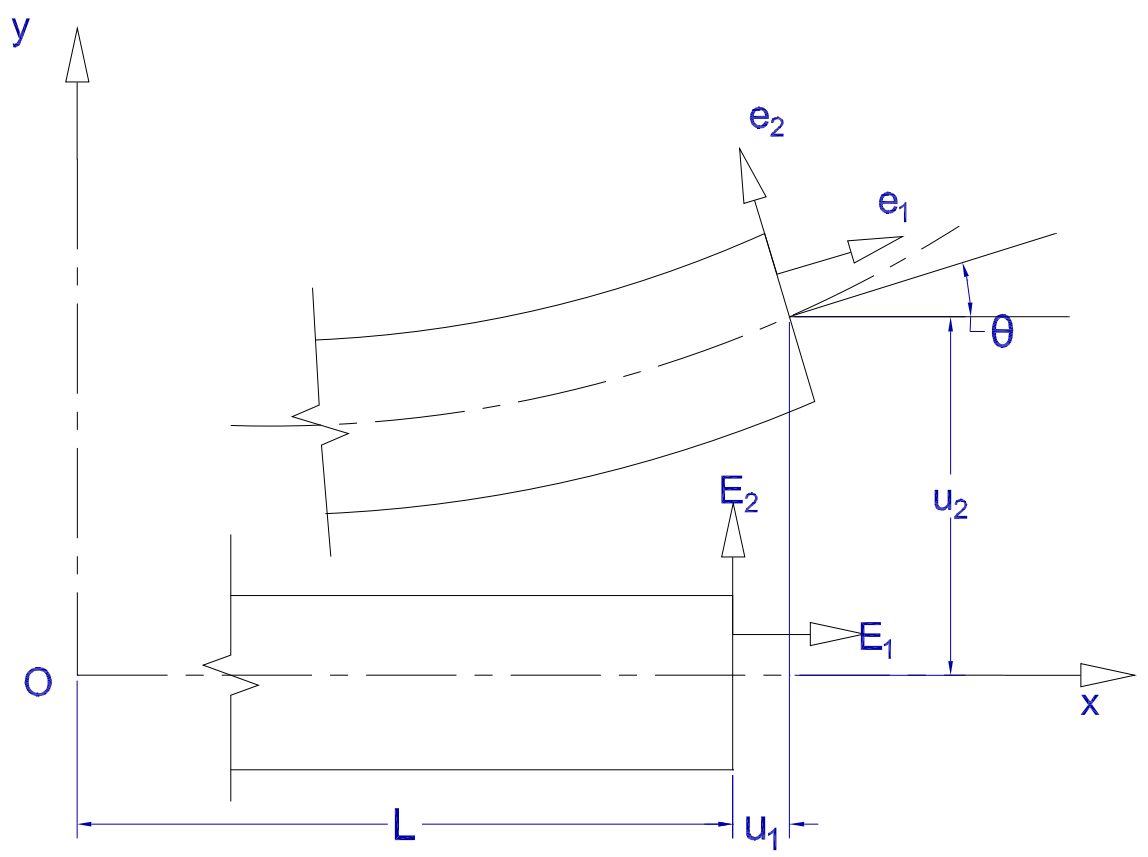

Figure 2.1: Kinematic assumption

During the beam motion, the beam deforms from its initial(straight) configuration to its current configuration shown in Figure 2.2. In a similar manner to the beam reference configuration, after deformation, the line of centroids is now a curve with position vector of a particle on the line of centroids denoted by $\boldsymbol{x}_{0}$. Locally attached to the Beam cross-section in the current configuration, we have a current 
or moving orthonormal frame $\boldsymbol{e}_{1}, \boldsymbol{e}_{2}$ and $\boldsymbol{e}_{3}$ with origin located on the current axis. $\boldsymbol{e}_{1}$ and $\boldsymbol{e}_{2}$ point in the direction of the principal axes of inertia in the current configuration, and $\boldsymbol{e}_{1}$ is normal to the cross-section in question (see also assumption $i$ above).

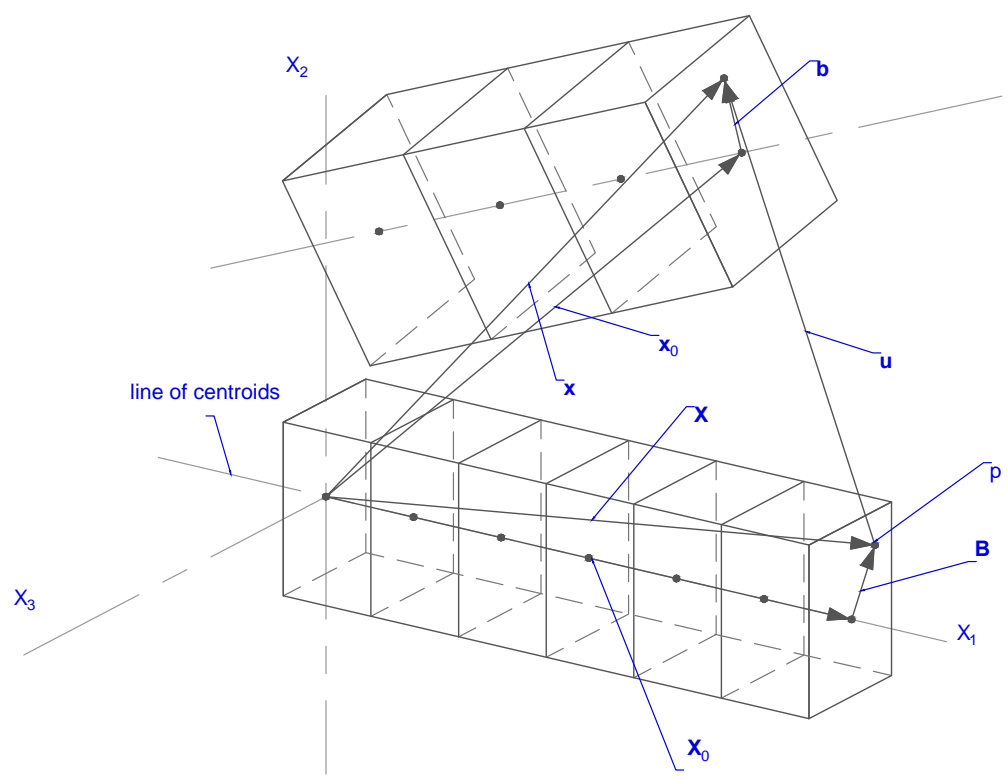

Figure 2.2: $3 \mathrm{D}$ representation of the coordinate system, including the reference and deformed configurations

Assuming the geometric shape of the beam cross-section is arbitrary, the initial position vector of an arbitrary material particle in the cross-section is defined as

$$
\boldsymbol{X}=\boldsymbol{X}_{0}\left(\boldsymbol{E}_{1}\right)+\boldsymbol{B}\left(\boldsymbol{E}_{2}, \boldsymbol{E}_{3}\right)
$$

where $\boldsymbol{A}$ is the position of the particle within the cross-section.

Taking the reference frame $\left(\boldsymbol{E}_{1}, \boldsymbol{E}_{2}\right.$ and $\left.\boldsymbol{E}_{3}\right)$ and the moving frame $\left(\boldsymbol{e}_{1}, \boldsymbol{e}_{2}\right.$ and $\left.\boldsymbol{e}_{3}\right)$ to coincide, we can introduce a one-parameter rotation tensor $\boldsymbol{\Lambda}$ to relate them. The rotation tensor $\boldsymbol{\Lambda}$ maps the base vectors of the reference frame onto the current frame, hence we may define the moving frame as a rotated reference frame (see Figure 2.3p.

The rotation tensor is a two-point tensor defined as

$$
\boldsymbol{\Lambda}=\boldsymbol{e}_{i} \otimes \boldsymbol{E}_{i}
$$




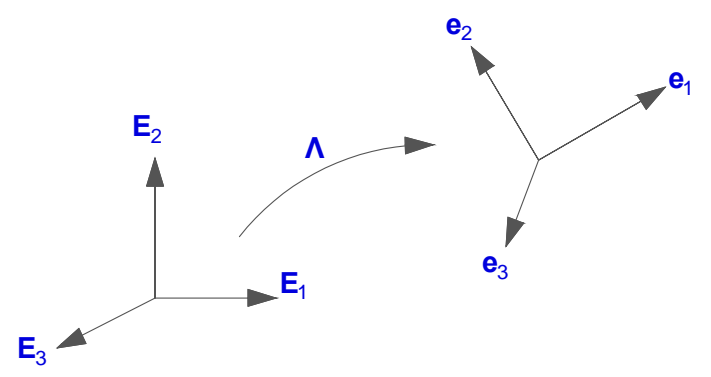

Figure 2.3: Rotation tensor $\Lambda$

and the reference and moving frame are related by the equation

$$
\boldsymbol{e}_{i}=\Lambda \boldsymbol{E}_{i}
$$

Here, $\boldsymbol{\Lambda}$ (computed by employing the Rodriguez formula in a subsequent section) belongs to the Lie group of proper orthogonal transformations, and is a linear operator with the following well known properties

$$
\boldsymbol{\Lambda}^{T} \boldsymbol{\Lambda}=\boldsymbol{\Lambda} \boldsymbol{\Lambda}^{T}=\boldsymbol{I} ; \quad \boldsymbol{\Lambda}^{-1}=\boldsymbol{\Lambda}^{T} ; \quad \operatorname{det} \boldsymbol{\Lambda}=1 .
$$

In the beam current configuration, the position vector of an arbitrary particle on the line of centroids is given by

$$
\boldsymbol{x}_{0}=\boldsymbol{X}_{0}(x)+\boldsymbol{u}(x)
$$

where the components of the displacement vector $\boldsymbol{u}$ are the cross-section translational degrees of freedom. The position of an arbitrary particle in the current configuration of the beam is given by

$$
\boldsymbol{x}=\boldsymbol{x}_{0}+\boldsymbol{b}
$$

where $\boldsymbol{b}$ is the position of the particle in the rotated cross-section.

$$
b=\Lambda B
$$


and $\boldsymbol{B}$ its counterpart in the reference configuration.

Hence the beam motion is completely defined by the position of its line of centroids and the orientation of the moving frame with respect to the reference frame. In matrix form, the motion may be written as

$$
\left\{\begin{array}{l}
x \\
y \\
z
\end{array}\right\}=\left\{\begin{array}{c}
X_{1} \\
0 \\
0
\end{array}\right\}+\left\{\begin{array}{l}
u_{1} \\
u_{2} \\
u_{3}
\end{array}\right\}+\left[\begin{array}{ccc}
\Lambda_{11} & \Lambda_{12} & \Lambda_{13} \\
\Lambda_{21} & \Lambda_{22} & \Lambda_{23} \\
\Lambda_{31} & \Lambda_{32} & \Lambda_{33}
\end{array}\right]\left\{\begin{array}{c}
0 \\
X_{2} \\
X_{3}
\end{array}\right\}
$$

\subsection{Large Rotations}

Working with finite rotations encountered in the geometrically exact formulation for the three-dimensional beam requires proper treatment of the rotational degrees of freedom. As opposed to small or infinitesimal rotations, finite three-dimensional rotations introduce most of the complexities of the model due to its nonlinear character.

Without going into the more general setting of manifolds, we present the main properties of the rotation group, and a brief discussion on the possible parametrization of three-dimensional finite rotations. For detailed reading on large rotations, see works by [2], [12], [59], and for application to beams and shells [31].

\subsubsection{Rotation group}

In geometry, rotation group is the group of all rotations about the origin of the threedimensional Euclidean space $\mathbb{R}^{3}$. Finite three-dimensional rotations can always be represented by an orthogonal tensor, say $\boldsymbol{\Lambda}$, an element of the $S 0$ (3) rotation group, which is a special three-dimensional orthogonal group containing information about the axis of rotation and the rotation itself.

$$
S 0(3):=\boldsymbol{\Lambda}: \mathbb{R}^{3} \rightarrow \mathbb{R}^{3} \mid \boldsymbol{\Lambda}^{T} \boldsymbol{\Lambda}=\boldsymbol{\Lambda} \boldsymbol{\Lambda}^{T}=I, \operatorname{det} \boldsymbol{\Lambda}=+1
$$

The rotation operator $\boldsymbol{\Lambda}$, transforms an orthonormal basis into another basis in the Euclidean space. Properties of the rotation group include:

(i) Every rotation possesses a unique inverse rotation

$$
\boldsymbol{\Lambda} \boldsymbol{\Lambda}^{-1}=\boldsymbol{\Lambda}^{-1} \boldsymbol{\Lambda}=\boldsymbol{I} \quad \forall \boldsymbol{\Lambda} \in S O(3) ;
$$


(ii) Product of three rotations is associative

$$
\boldsymbol{\Lambda}_{1}\left(\boldsymbol{\Lambda}_{2} \boldsymbol{\Lambda}_{3}\right)=\left(\boldsymbol{\Lambda}_{1} \boldsymbol{\Lambda}_{2}\right) \boldsymbol{\Lambda}_{3} \quad \forall \boldsymbol{\Lambda}_{1}, \boldsymbol{\Lambda}_{2}, \boldsymbol{\Lambda}_{3} \in S 0(3)
$$

(iii) Another rotation is the result of a composition of two rotations

$$
\boldsymbol{\Lambda}_{1} \boldsymbol{\Lambda}_{2}=\boldsymbol{\Lambda}_{3} \quad \forall \boldsymbol{\Lambda}_{1}, \boldsymbol{\Lambda}_{2} \in S 0(3) \text { and } \boldsymbol{\Lambda}_{3} \in S 0(3) ;
$$

(iv) The identity matrix is a neutral element

$$
\boldsymbol{I} \boldsymbol{\Lambda}=\boldsymbol{I} \boldsymbol{\Lambda}=\boldsymbol{\Lambda} \quad \forall \boldsymbol{\Lambda} \in S O(3)
$$

(v) In general, the product is non-commutative

$$
\boldsymbol{\Lambda}_{1} \boldsymbol{\Lambda}_{2} \neq \boldsymbol{\Lambda}_{2} \boldsymbol{\Lambda}_{1} \quad \forall \boldsymbol{\Lambda}_{1}, \boldsymbol{\Lambda}_{2} \in S 0(3)
$$

(vi) In general, rotations are also non-additive since the rotation set is not a linear space

\subsubsection{Parametrization of Finite Rotations}

The choice of parameters in terms of degrees of freedom describing the rotation is known as parametrization of the rotation tensor. Several forms are used in literature for this parametrization including vector-like parametrization of which the EulerRodriguez parametrization belongs, and the non vector-like parametrization which includes Euler angles and quaternions among others.

The six orthogonality conditions relate the nine components of the rotation matrix, hence the representation of $\boldsymbol{\Lambda}$ can be reduced to only 3 parameters. However, it has been established by [59] that a unique global representation of finite rotations based on only three parameters does not exist, and a minimum of five parameters is required. The work of [1], based on the quaternion method shows that a fourparameter representation of finite rotations is also potentially useful for practical purposes, regardless of the fact that it is not strictly 1-1 but rather, a 2-1 representation.

The choice of the rotation parametrization is influenced mainly by the specific requirements of its intended application, and also by the theoretical and computational issues associated with them. In this work, we make use of the rotation vector $\boldsymbol{\theta}$, and upon application of the Rodrigues formula, recover the rotation matrix $\boldsymbol{\Lambda}$. 


\section{Euler-Rodrigues parametrization}

The Rodrigues formula which expresses the rotation tensor in terms of the rotation vector can be derived from a geometric or differential approach. The basis for the geometric derivation is Euler's theorem which states that any arbitrary displacement of a rigid body that leaves one point fixed is a rotation about the unit vector of the axis of rotation. Additional information on the geometrical representation of the formula can be found in [21] and [31]. From a physical point of view, threedimensional rotations can be seen as two-dimensional rotations which take place in the plane orthogonal to the suitably chosen axis of rotation.

From the Rodrigues formula, the rotation tensor $\boldsymbol{\Lambda}$, expressed in terms of the rotation vector is given below as

$$
\boldsymbol{\Lambda}(\boldsymbol{\theta})=\cos \theta \boldsymbol{I}+\frac{\sin \theta}{\theta} \boldsymbol{\Theta}+\frac{1-\cos \theta}{\theta^{2}}(\boldsymbol{\theta} \otimes \boldsymbol{\theta})
$$

With components $\theta_{1}, \theta_{2}$, and $\theta_{3}$ in a Cartesian system,

$$
\theta=\|\boldsymbol{\theta}\|=\sqrt{\theta_{1}^{2}+\theta_{2}^{2}+\theta_{3}^{2}}
$$

$\boldsymbol{I}$ is a $3 \times 3$ unit matrix, and $\boldsymbol{\Theta}$ is the skew-symmetric tensor associated with the rotational vector $\boldsymbol{\theta}$ which is the axial vector.

$$
\begin{gathered}
\boldsymbol{\Theta}=-\boldsymbol{\Theta}^{T}=\left[\begin{array}{ccc}
0 & -\theta_{3} & \theta_{2} \\
\theta_{3} & 0 & -\theta_{1} \\
-\theta_{2} & \theta_{1} & 0
\end{array}\right] \quad\left\{\begin{array}{l}
\theta_{1} \\
\theta_{2} \\
\theta_{3}
\end{array}\right\} \\
\boldsymbol{\Theta} \boldsymbol{h}=\boldsymbol{\theta} \times \boldsymbol{h} \quad \forall \boldsymbol{h} \in \mathbb{R}^{3}
\end{gathered}
$$

An alternative form of the Rodrigues formula, which is a theoretically more convenient function of $\boldsymbol{\theta}$, possesses the advantage to simplify differentiation of the rotation tensor $\boldsymbol{\Lambda}$. The exponential map has become a favourite for implementation and will be used to construct the admissible variation of the orthogonal tensor of finite rotations $\boldsymbol{\Lambda}$ :

$$
\boldsymbol{\Lambda}=\exp [\boldsymbol{\Theta}]
$$

A closed form solution of the exponential map above has been derived in literature [?] and is given as

$$
\boldsymbol{\Lambda}=\exp [\boldsymbol{\Theta}]=\boldsymbol{I}+\frac{\sin \theta}{\theta} \boldsymbol{\Theta}+\frac{1-\cos \theta}{\theta^{2}} \boldsymbol{\Theta}^{2}
$$




\subsubsection{Configuration Space and Compound Rotations}

Rotation operators define rotations as seen in previous sections, and the components of the rotation operator depends on the chosen frame of reference. Known as material and spatial reference frames in finite deformation analysis, expressing the components with respect to a fixed reference frame is known as material, and is referred to as spatial when expressed with respect to a moving frame attached to the rotating body. Applying a series of rotations, say $\boldsymbol{\Lambda}_{i}(i=1,2,3)$ making up a compound rotation $\boldsymbol{\Lambda}_{T}$, to an arbitrary vector $\boldsymbol{a} \in \mathbb{R}^{3}$ can be defined by the two (material and spatial) descriptions which are equivalent.

In the spatial description, the rotated vector $\boldsymbol{a}_{T} \in \mathbb{R}^{3}$ obtained upon application of the compound rotation $\boldsymbol{\Lambda}_{T} \in S 0(3)$, is the result of the consecutive application of the rotations $\boldsymbol{\Lambda}_{i} \in S 0(3)$ on the previous rotated vector.

$$
\boldsymbol{a}_{T}=\boldsymbol{\Lambda}_{3}\left(\boldsymbol{\Lambda}_{2}\left(\boldsymbol{\Lambda}_{1} \boldsymbol{a}\right)\right)=\boldsymbol{\Lambda}_{T} \boldsymbol{a}
$$

Here, the inverse multiplicative rule applies for the composition of rotations.

In the material description, we have

$$
\boldsymbol{a}_{T}=\boldsymbol{\Lambda}_{1}\left(\boldsymbol{\Lambda}_{2}\left(\boldsymbol{\Lambda}_{3} \boldsymbol{a}\right)\right)=\boldsymbol{\Lambda}_{T} \boldsymbol{a}
$$

The direct multiplicative rule applies, with the important distinction here that the rotation tensors $\boldsymbol{\Lambda}_{i}$ are expressed in the new (rotated) or updated reference frame affected by the previous rotation.

According to Equations 2.3.13 and 2.3.14, applying a rotation increment results in a new compound rotation and we can define the material and spatial descriptions of this compound rotation.

For a material incremental rotation vector $\vartheta$ the compound rotation tensor is given by

$$
\boldsymbol{\Lambda}_{T}=\boldsymbol{\Lambda} \exp [\overline{\boldsymbol{\vartheta}}]=\boldsymbol{\Lambda} \boldsymbol{\Lambda}^{m a t}
$$

and for a spatial incremental rotation vector $\boldsymbol{\theta}$, we have

$$
\boldsymbol{\Lambda}_{T}=\exp [\overline{\boldsymbol{\theta}}] \boldsymbol{\Lambda}=\boldsymbol{\Lambda}^{s p} \boldsymbol{\Lambda}
$$

where $\boldsymbol{\Lambda}^{\text {mat }} \in S O(3)$ and $\boldsymbol{\Lambda}^{s p} \in S O(3)$ are material and spatial forms respectively of the incremental rotation operator, and $\overline{\boldsymbol{\vartheta}}$ and $\overline{\boldsymbol{\theta}}$ are the skew-symmetric tensors whose axial vectors are $\boldsymbol{\vartheta}$ and $\boldsymbol{\theta}$ respectively.

From Equations 2.3.15 and 2.3.16 above, and taking into account properties 
of the rotation group, we recover the following relationships between spatial and material descriptions of the rotation operators and incremental rotation tensors as

$$
\begin{gathered}
\boldsymbol{\Lambda}^{s p}=\boldsymbol{\Lambda} \boldsymbol{\Lambda}^{m a t} \boldsymbol{\Lambda}^{T} \\
\overline{\boldsymbol{\theta}}=\boldsymbol{\Lambda} \overline{\boldsymbol{\vartheta}} \boldsymbol{\Lambda}^{T}
\end{gathered}
$$

\subsubsection{Rotation parameters}

For the linearisation of the nonlinear equations of motion, we will need admissible variations of the various rotation quantities we have introduced. Here we present material and spatial descriptions of the variations and relate them with the well known push-forward and pull-back operations.

In the material description, taking $\delta \boldsymbol{\Psi}$ as the material skew symmetric tensor representing infinitesimal rotations, we have from Equation 2.3.15

$$
\delta \boldsymbol{\Lambda}=\left.\frac{d}{d \epsilon} \boldsymbol{\Lambda} \exp (\epsilon \delta \boldsymbol{\Psi})\right|_{\epsilon=0}=\boldsymbol{\Lambda} \delta \boldsymbol{\Psi}
$$

In a similar manner, with $\delta \boldsymbol{W}$ as the spatial skew symmetric rotation tensor representing infinitesimal rotation, we have from Equation 2.3.16

$$
\delta \boldsymbol{\Lambda}=\left.\frac{d}{d \epsilon} \exp (\epsilon \delta \boldsymbol{W}) \boldsymbol{\Lambda}\right|_{\epsilon=0}=\delta \boldsymbol{W} \boldsymbol{\Lambda}
$$

from Equation 2.3.19 and 2.3.20 above, we obtain the push forward and pull back operations

$$
\delta \boldsymbol{W}=\boldsymbol{\Lambda} \delta \boldsymbol{\Psi} \boldsymbol{\Lambda}^{T} \quad \text { and } \quad \delta \boldsymbol{\Psi}=\boldsymbol{\Lambda}^{T} \delta \boldsymbol{W} \boldsymbol{\Lambda}
$$

and their corresponding axial vectors

$$
\delta \boldsymbol{w}=\boldsymbol{\Lambda} \delta \boldsymbol{\psi} \quad \text { and } \quad \delta \boldsymbol{\psi}=\boldsymbol{\Lambda}^{T} \delta \boldsymbol{w}
$$

The variations can also be computed in terms of the rotation vector (vector like rotation parameters). Considering the material and spatial total rotation vectors $\vartheta$ and $\theta$ respectively which can be used to parametrize the rotation tensor $\Lambda$, we have

$$
\boldsymbol{\Lambda}=\exp [\overline{\boldsymbol{\vartheta}}]=\exp [\overline{\boldsymbol{\theta}}]
$$

The material total rotation vector is that associated with the fixed axis which remains fixed during the rotation but the incremental rotation vector $\boldsymbol{\psi}$ is associated 
with the follower axis which is rotated by the previous rotation. In the material description, the compound total rotation vector $\boldsymbol{\vartheta}+\delta \boldsymbol{\vartheta}$, where $\delta \boldsymbol{\vartheta}$ is the additive increment of $\boldsymbol{\vartheta}$ parametrizing $\boldsymbol{\Lambda}$, we have

$$
\exp [\overline{\boldsymbol{\vartheta}}+\delta \overline{\boldsymbol{\vartheta}}]=\exp [\overline{\boldsymbol{\vartheta}}] \exp [\delta \boldsymbol{\Psi}]
$$

Rearranging Equation 2.3.24, taking the directional derivative, and using the Rodrigues' formula, the linearized relation between $\delta \vartheta$ and $\delta \psi$ is obtained from

$$
\begin{aligned}
\exp [\overline{\boldsymbol{\vartheta}}+\epsilon \delta \overline{\boldsymbol{\vartheta}}]=\exp [\overline{\boldsymbol{\vartheta}}] \exp [\epsilon \delta \boldsymbol{\Psi}] \\
\delta \boldsymbol{\psi}=D[\boldsymbol{\Lambda}(\epsilon, \boldsymbol{\psi})] \\
=\left.\frac{d}{d \epsilon}[\exp [\epsilon \delta \boldsymbol{\Psi}]]\right|_{\epsilon=0} \\
=\left.\frac{d}{d \epsilon}[\exp [-\overline{\boldsymbol{\vartheta}}] \exp [\overline{\boldsymbol{\vartheta}}+\epsilon \delta \overline{\boldsymbol{\vartheta}}]]\right|_{\epsilon=0}
\end{aligned}
$$

Similarly in the spatial description, for the compound total rotation vector, $\boldsymbol{\theta}+$ $\delta \boldsymbol{\theta}$, we have

$$
\begin{aligned}
\exp [\overline{\boldsymbol{\theta}}+\epsilon \delta \overline{\boldsymbol{\theta}}]=\exp [\epsilon \delta \boldsymbol{W}] \exp [\overline{\boldsymbol{\theta}}] \\
\delta \boldsymbol{w}=D[\boldsymbol{\Lambda}(\epsilon, \boldsymbol{w})] \\
=\left.\frac{d}{d \epsilon}[\exp [\epsilon \delta \boldsymbol{w}]]\right|_{\epsilon=0} \\
=\left.\frac{d}{d \epsilon}[\exp [\overline{\boldsymbol{\theta}}+\epsilon \delta \overline{\boldsymbol{\theta}}] \exp [-\overline{\boldsymbol{\theta}}]]\right|_{\epsilon=0}
\end{aligned}
$$

\subsubsection{Spatial derivative (Curvature)}

Following Reissner's approach [51] and also [29], to recover the beam curvature measure which will be used to express the strain measures, we use $(\bullet)_{s}$ to denote the derivative with respect to the arc-length coordinate of the beam.

From the orthogonality condition, $\boldsymbol{\Lambda}^{T} \boldsymbol{\Lambda}=\boldsymbol{I}$, we have

$$
\boldsymbol{\Lambda}^{T}{ }_{s} \boldsymbol{\Lambda}+\boldsymbol{\Lambda}^{T} \boldsymbol{\Lambda},{ }_{s}=0
$$


recalling the skewness condition,

$$
-\boldsymbol{\Lambda}^{T}{ }_{s} \boldsymbol{\Lambda}=\boldsymbol{\Lambda}^{T} \boldsymbol{\Lambda},{ }_{s}=-\left(\boldsymbol{\Lambda}^{T} \boldsymbol{\Lambda},{ }_{s}\right)^{T}
$$

the material and spatial curvature tensors (skew-symmetric) $\boldsymbol{K}$ and $\boldsymbol{K}^{\varphi}$ respectively are given as

$$
\boldsymbol{K}=\boldsymbol{\Lambda}^{T} \boldsymbol{\Lambda},{ }_{s} \quad \text { and } \quad \boldsymbol{K}^{\varphi}=\boldsymbol{\Lambda}, \boldsymbol{\Lambda}^{T}
$$

\subsection{Strain measures}

Required for the beam principle of virtual work, here we present the strain measures. Introduced earlier, the deformed Beam configuration is completely defined by the position of its line of centroids and the orientation of the local Cartesian triad. The motion can be mathematically described by the point mapping between initial and current positions as

$$
\boldsymbol{x}^{\varphi}=\phi(\boldsymbol{x})
$$

and the isometric transformation of the cross-section normal $\boldsymbol{E}_{1}$, denoted by $\boldsymbol{g}$ into its counterpart in the current configuration $\boldsymbol{e}_{1}$, denoted by $\boldsymbol{a}$

$$
a=\Lambda g
$$

where $\boldsymbol{a}$ and $\boldsymbol{g}$ are unit vectors and $\boldsymbol{\Lambda}$ is an orthogonal tensor. Following Reissner's approach [49], the translation and rotational strain measures are given respectively as

$$
\boldsymbol{\epsilon}=\boldsymbol{\Lambda}^{T} \phi{ }_{s}-\boldsymbol{g}
$$

the components of which are the axial and shear strains, and a skew-symmetric tensor for the bending strains

$$
\boldsymbol{K}=\boldsymbol{\Lambda}^{T} \boldsymbol{\Lambda},{ }_{s}
$$

Here for simplicity, the beam is assumed to lie along the $\mathrm{x}$-axis, hence

$$
\boldsymbol{g}=\left\{\begin{array}{l}
1 \\
0 \\
0
\end{array}\right\}=\boldsymbol{E}_{1}
$$


The strain objects above can also be represented in their spatial forms by applying the push-forward operations

$$
\begin{aligned}
\boldsymbol{\epsilon}^{\varphi} & =\boldsymbol{\Lambda} \boldsymbol{\epsilon} \\
& =\boldsymbol{\Lambda}\left(\boldsymbol{\Lambda}^{T} \boldsymbol{\phi},{ }_{s}-\boldsymbol{g}\right) \\
& =\boldsymbol{\phi},{ }_{s}-\boldsymbol{a}
\end{aligned}
$$

and

$$
\begin{aligned}
\boldsymbol{K}^{\varphi} & =\boldsymbol{\Lambda} \boldsymbol{K} \boldsymbol{\Lambda}^{T} \\
& =\boldsymbol{\Lambda}\left(\boldsymbol{\Lambda}^{T} \boldsymbol{\Lambda},{ }_{s}\right) \boldsymbol{\Lambda}^{T} \\
& =\boldsymbol{\Lambda},{ }_{s} \boldsymbol{\Lambda}^{T}
\end{aligned}
$$

The derivative of the rotation matrix with respect to the arc-length coordinate required for computations is given in 62 as

$$
\boldsymbol{\Lambda},{ }_{s}=\boldsymbol{\Theta},_{s} \boldsymbol{\Lambda}
$$

where $\boldsymbol{\Theta},_{s}$ is the skew-symmetric matrix for the derivatives of the rotation vector. In matrix form,

$$
\boldsymbol{\Theta},,_{s}=\left[\begin{array}{ccc}
0 & -\theta_{3}, s & \theta_{2}, s \\
\theta_{3, s} & 0 & -\theta_{1}, s \\
-\theta_{2},{ }_{s} & \theta_{1},{ }_{s} & 0
\end{array}\right]
$$

Virtual strains $\delta \boldsymbol{\epsilon}$ and $\delta \boldsymbol{\kappa}$ required to develop virtual work equations for the beam are given in 31 ]

$$
\delta \boldsymbol{\epsilon}=\boldsymbol{\Lambda}^{T} \delta \boldsymbol{\phi},{ }_{s}+\boldsymbol{\Lambda}^{T} \boldsymbol{\phi},{ }_{s} \times \delta \boldsymbol{\psi}
$$

and

$$
\delta \boldsymbol{\kappa}=\delta \boldsymbol{\psi},{ }_{s}+\boldsymbol{\kappa} \times \delta \boldsymbol{\psi}
$$




\subsection{Equilibrium and virtual work equations}

Three conservation principles should be satisfied in any motion within classical continuum mechanics which are conservation of mass, and the balance of linear and angular momenta. For quasi static applications in the reference configuration, the balance laws are given as

$$
\begin{gathered}
\int_{\partial v} \boldsymbol{t} \mathrm{d} a+\int_{v} \boldsymbol{b} \mathrm{d} v=0 \\
\int_{\partial v} \boldsymbol{x} \times \boldsymbol{t} \mathrm{d} a+\int_{v} \boldsymbol{x} \times \boldsymbol{b} \mathrm{d} v=0
\end{gathered}
$$

where $\boldsymbol{n}$ is the outward unit normal to the boundary of a body with volume $v$, boundary $\partial v$, under the action of body forces per unit volume $b$, and traction forces per unit area $\boldsymbol{t}$ acting on the boundary. Introducing the First Piola-Kirchhoff stress tensor, $\boldsymbol{P}$, according to the Cauchy postulate, we have $\boldsymbol{t}=\boldsymbol{P} \boldsymbol{n}$ (see [57]).

Having presented the strain measures in Equation 2.4.3 and Equation 2.4.4 above, the potential energy of the beam can be written as

$$
\boldsymbol{\Pi}(\boldsymbol{\phi}, \boldsymbol{\theta})=\frac{1}{2} \int_{L}(\boldsymbol{\epsilon} \cdot \boldsymbol{n}+\boldsymbol{\kappa} \cdot \boldsymbol{m}) \mathrm{d} L-\boldsymbol{\Pi}_{e x t}
$$

where $\boldsymbol{n}$ and $\boldsymbol{m}$ are stress resultants and couples.

Assuming the strains are small, which is true for most engineering structures even those undergoing large deflections and rotations, the material behaviour can be described by the linear Elastic theory. For these, we have the constitutive matrices

$$
\boldsymbol{C}=\left[\begin{array}{ccc}
E A & 0 & 0 \\
0 & G A_{2} & 0 \\
0 & 0 & G A_{3}
\end{array}\right] \quad \boldsymbol{D}=\left[\begin{array}{ccc}
G J & 0 & 0 \\
0 & E I_{2} & 0 \\
0 & 0 & E I_{3}
\end{array}\right]
$$

Here, $\boldsymbol{C}$ and $\boldsymbol{D}$ are constitutive matrices of the relations between translational strains and cross-sectional forces, and rotational strains and cross-sectional moments respectively. $E$ and $G$ are elastic and shear moduli of the material respectively. The shear areas in the principal directions of the cross section (1 and 2) are represented by $A_{2}$ and $A_{3} . I_{2}$ and $I_{3}$ are the cross sectional inertial moments about the principal directions 1 and 2 respectively, $A$ is the cross-sectional area, and $J$ is the torsional inertial moment of the cross-section.

Hence we have the stress resultants

$$
\boldsymbol{n}=\boldsymbol{C} \epsilon, \quad \boldsymbol{m}=\boldsymbol{D} \kappa
$$


In the current configuration, the stress resultants can also be obtained after performing the relevant operations as

$$
\boldsymbol{n}^{\phi}=\boldsymbol{\Lambda n}, \quad \boldsymbol{m}^{\phi}=\boldsymbol{\Lambda} \boldsymbol{m}
$$

As explained in [31], by performing the directional derivative of the potential energy in the direction of virtual displacements and rotations, the virtual work equation is obtained as

$$
\delta \boldsymbol{\Pi}(\boldsymbol{\psi}, \boldsymbol{\theta}) \cdot(\delta \boldsymbol{\psi}, \delta \boldsymbol{\theta})=\int_{L}(\delta \boldsymbol{\epsilon} \cdot \boldsymbol{n}+\delta \boldsymbol{\kappa} \cdot \boldsymbol{m}) \mathrm{d} L-\boldsymbol{\Pi}_{e x t}=0
$$

The first term on the right hand side of the above functional gives rise to the residual, and the finite element method will be applied to solve Equation 2.5.7.

Required by the newton-raphson solution procedure, the consistent linearization of the quantities introduced above, and the virtual work equation, are performed using the directional derivative in the direction of the displacements and rotations.

\subsection{Finite Element Implementation}

The finite element solution of this non-linear problem requires the use of the NewtonRaphson iterative process, applying the external load in a series of increments, and iterating within each load increment till the specified tolerance is reached. We make use of the 2-node linear finite element for the finite element approximations. Extensive information on these topics is available in e.g. [15], 62, [20].

Considering the solution of a set of nonlinear algebraic equations:

$$
\boldsymbol{R}(\boldsymbol{x})=\mathbf{0}
$$

the iterative process uses a solution estimate $\boldsymbol{x}_{k}$ at iteration $k$ to obtain a new value $\boldsymbol{x}_{k+1}=\boldsymbol{x}_{k}+\boldsymbol{u}$ in terms of an increment $\boldsymbol{u}$ by establishing the linear approximation

$$
\boldsymbol{R}\left(\boldsymbol{x}_{k+1}\right) \approx \boldsymbol{R}\left(\boldsymbol{x}_{k}\right)+D \boldsymbol{R}\left(\boldsymbol{x}_{k}\right)[\boldsymbol{u}]=\mathbf{0}
$$

employing the directional derivative and obtaining the tangent matrix $\boldsymbol{K}$, a linear set of equations is solved at each Newton-Raphson iteration as

$$
\boldsymbol{K}\left(\boldsymbol{x}_{k}\right) \boldsymbol{u}=-\boldsymbol{R}\left(\boldsymbol{x}_{k}\right) ; \quad \boldsymbol{x}_{k+1}=\boldsymbol{x}_{k}+\boldsymbol{u}
$$

The algorithm is shown below [11]:

- Input geometry, material properties, and solution parameters 
- Initialize $\boldsymbol{F}=\mathbf{0}, \boldsymbol{x}=\boldsymbol{X}, \boldsymbol{R}=\mathbf{0}$

- Find initial $\boldsymbol{K}$

- Loop over load increments

- Find $\Delta \boldsymbol{F}$, set $\boldsymbol{F}=\boldsymbol{F}+\Delta \boldsymbol{F}$

- set $\boldsymbol{R}=\boldsymbol{R}-\Delta \boldsymbol{F}$

- Do While $(\|\boldsymbol{R}\| /\|\boldsymbol{F}\|>$ tolerance $)$

* solve $\boldsymbol{K} \boldsymbol{u}=-\boldsymbol{R}$

* update $\boldsymbol{x}=\boldsymbol{x}+\boldsymbol{u}$

* find $\boldsymbol{T}$ and $\boldsymbol{K}$

* find $\boldsymbol{R}=\boldsymbol{T}-\boldsymbol{F}$

- End Do

- End Loop

For the finite element approximation, the beam is divided into elements and shape functions are introduced and used to construct the element degrees of freedom. This is given by

$$
\left\{\begin{array}{l}
\boldsymbol{u}^{e} \\
\boldsymbol{\theta}^{e}
\end{array}\right\}=\sum_{a=1}^{n}\left[\begin{array}{cc}
N_{a} & 0 \\
0 & N_{a}
\end{array}\right]\left\{\begin{array}{c}
\boldsymbol{u}_{a} \\
\boldsymbol{\theta}_{a}
\end{array}\right\}
$$

where $n$ is the number of element nodes. For a 2-noded element, we have that

$$
\begin{aligned}
N_{1} & =\frac{1-\xi}{2} \\
N_{2} & =\frac{1+\xi}{2} \\
\text { and } \quad d x & =\frac{l_{e}}{2} d \xi
\end{aligned}
$$

This geometrically exact model is well known, but other than the basics of the model (kinematics, parametrization of finite rotations, strain measures and equilibrium equations), the quantities and expressions required for finite element implementation were the result of our work. Hence the focus on ensuring quadratic convergence in the numerical examples, to show accuracy of our work as well as excellent agreement with results shown in the examples. 

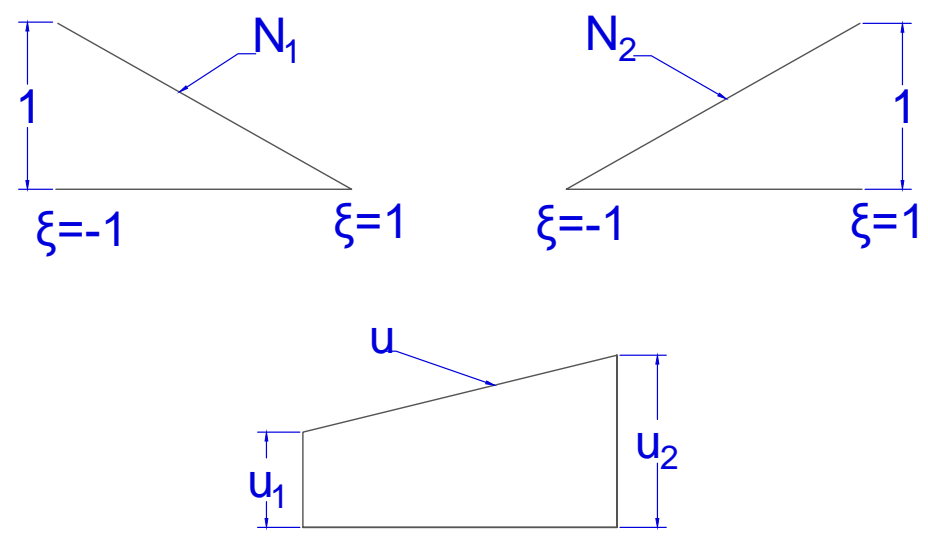

Figure 2.4: 2 node linear shape functions and interpolation

The codes were written in $\boldsymbol{C}++$ as separate subroutines in MPAP (Multi Physics Analysis Program) developed and maintained by the team of researchers at Swansea University.

\subsection{Numerical Results}

Here, we present benchmark tests used in literature to test and illustrate the performance of the model, and also compare the obtained results with available analytical solutions and other formulations. In this section, we present small deflection check, large displacement and rotation tests, buckling of a cantilever beam to determine its critical load, Lee frame, and roll-up of a cantilever beam. These problems are standard tests for the performance of the model in both the small and large deformation range, as well as stability analysis. The spatial problem chosen is the $45^{\circ}$ cantilever bend, as it offers a full three dimensional setting to test the ability of the model to handle large displacements and rotations. 


\subsubsection{Small deflection test of a Cantilever Beam}

In this standard example, we present an initially straight Cantilever Beam with an applied load at the free end. This example tests the behaviour of the model for small displacements and rotations, where we should recover the linear response. The Cantilever Beam has length $L=10 \mathrm{~m}$, axial stiffness $E A=1.2 \times 10^{6} \mathrm{~N}$, shear stiffness $G A=8.4 \times 10^{4} \mathrm{~N}$, bending stiffness $E I=4 \times 10^{4} \mathrm{Nm}^{2}$. Its free end is loaded with a vertical force $P=3 N$ and the tip deflection and rotation for meshes with increasing number of elements are shown in Table 2.1, and the convergence table is shown in Table 2.2 . For a sufficiently small value of load $P$, the expected linear response of the beam is

$$
\begin{gathered}
v_{\max }=\frac{P L^{3}}{3 E I}=0.025 \mathrm{~m} \\
\theta_{\max }=\frac{P L^{2}}{2 E I}=3.75 \times 10^{-3}
\end{gathered}
$$

which matches the result obtained from simulation, shown in Table 2.1, and Figure 2.6 shows the beam configuration after deformation for various load levels.

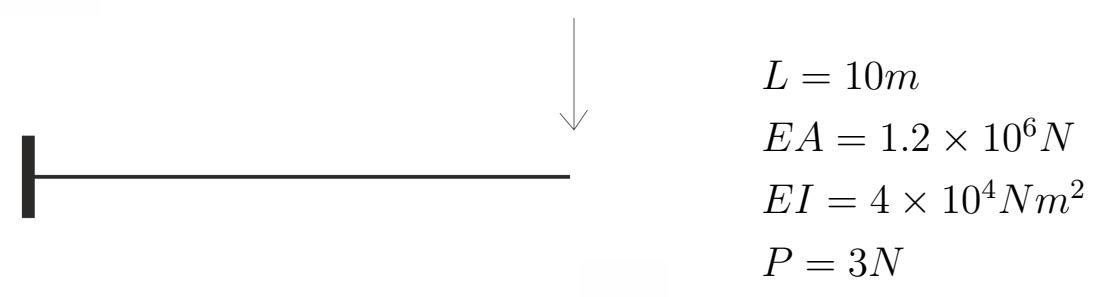

Figure 2.5: Geometry

\begin{tabular}{ccc}
\hline Number of elements & Tip deflection $(\mathrm{m})$ & Tip rotation \\
\hline 1 & 0.0191 & $3.75 \times 10^{-3}$ \\
5 & 0.0251 & $3.75 \times 10^{-3}$ \\
10 & 0.0253 & $3.75 \times 10^{-3}$ \\
20 & 0.0253 & $3.75 \times 10^{-3}$ \\
\hline
\end{tabular}

Table 2.1: Comparison of results for the small deflection case

\subsubsection{Large displacement and rotation test of a Cantilever Beam}

For performance test in the large deformation range, we consider an initially straight Cantilever beam loaded with bending and twisting moments $M$ and $T$ respectively 
at its free end. The Beam has length $L=10 \mathrm{~m}$, bending and torsional stiffness $E I=4 \times 10^{4} \mathrm{Nm}^{2}$ and $10^{3} \mathrm{Nm}^{2}$ respectively. Axial stiffness $E A=1.2 \times 10^{6} \mathrm{~N}$ and shear stiffness $G A=8.4 \times 10^{4} \mathrm{~N}$.

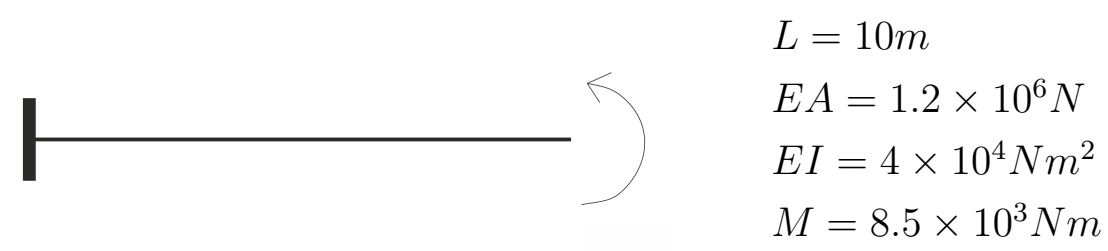

Figure 2.7: Geometry

From classical Euler formula for pure beam bending, the exact solution for the deformed beam is a part of a circle of radius $r=\frac{E I}{M}$. Based on this, the vertical and
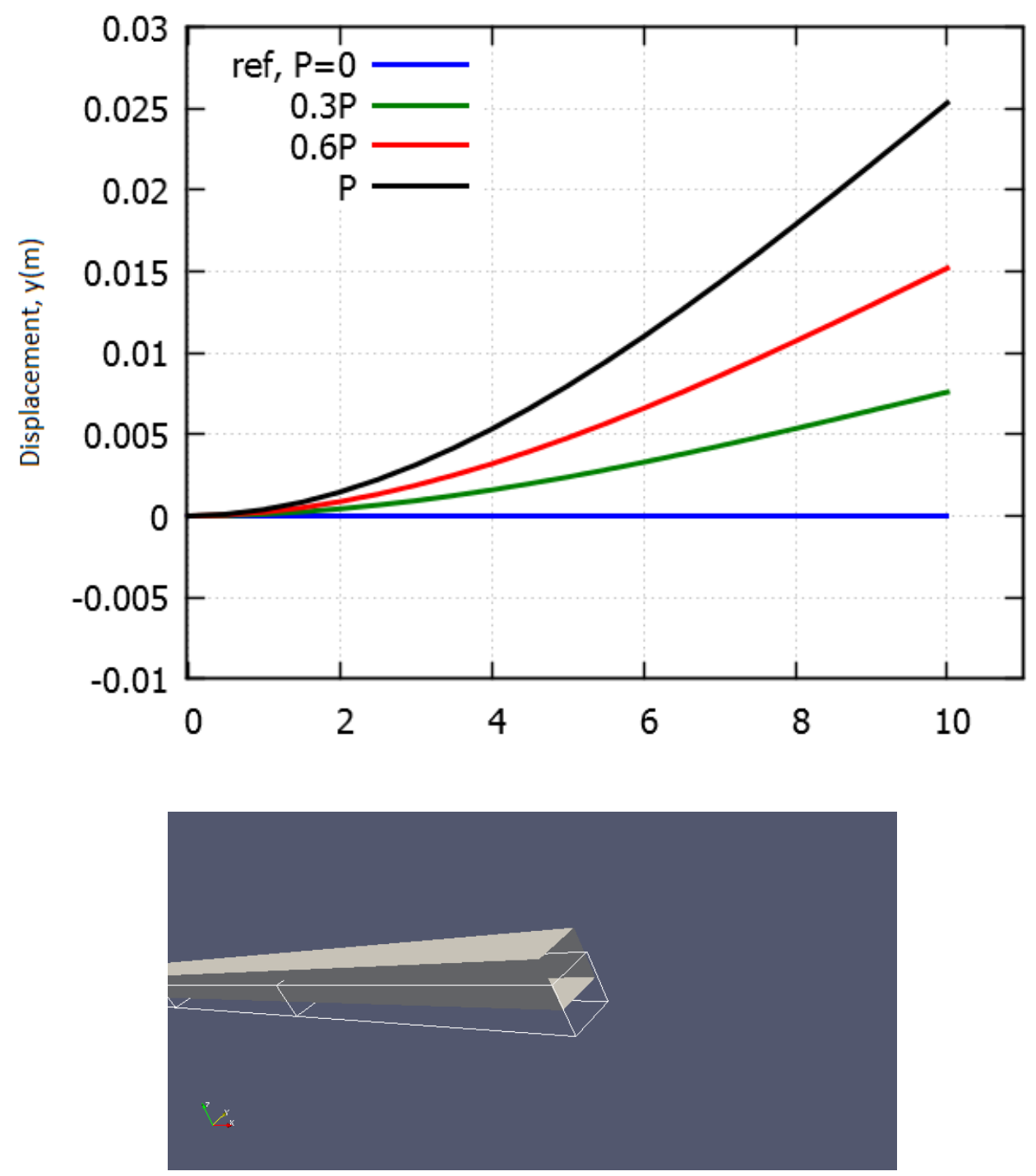

Figure 2.6: Deflection under applied load, $P$ at the free end 


\begin{tabular}{c|cc}
\hline \multirow{2}{*}{ iteration } & \multicolumn{2}{|c}{10 load steps } \\
\hline 1 & $t_{n-1}$ & $t_{n}$ \\
2 & $3 \times 10^{-} 1$ & $3 \times 10^{-} 1$ \\
3 & $8.104 \times 10^{-} 2$ & $8.104 \times 10^{-} 2$ \\
& $4.99 \times 10^{-} 10$ & $6.034 \times 10^{-} 10$ \\
\hline
\end{tabular}

Table 2.2: Convergence table for 10 load steps

horizontal components of displacement ( $u$ and $v$ ), and the rotation $\theta$ of the free end are

$$
\theta=\frac{M L}{E I}, \quad u=l-\frac{l}{\theta} \tan \frac{\theta}{2}(1+\cos \theta), \quad v=\frac{l}{\theta} \tan \frac{\theta}{2} \sin \theta
$$

The finite element mesh consists of twenty 2-noded elements, and a moment $M_{\text {max }}=8.5 \times 10^{3} \mathrm{Nm}$ is applied to the beam free end. Table 2.3 compares rotation and displacement vales for half-load and full-load levels, and Figure 2.8 shows the deflected shape of the beam.

\begin{tabular}{c|ccc|ccc}
\hline & \multicolumn{3}{|c}{$0.5 M_{\max }$} & \multicolumn{3}{c}{$M_{\max }$} \\
& $u(m)$ & $v(m)$ & $\theta$ & $u(m)$ & $v(m)$ & $\theta$ \\
\hline Analytic & 1.778 & 4.831 & 1.0625 & 5.999 & 7.182 & 2.125 \\
Present & 1.777 & 4.832 & 1.0625 & 5.997 & 7.186 & 2.125 \\
\hline
\end{tabular}

Table 2.3: Comparison of results for the large deflection case

For the case of a twisting moment $T$, from beam theory, for a beam of uniform cross-section along its length,

$$
\theta=\frac{T L}{G J}
$$

where $\theta=$ angle of twist in radians.

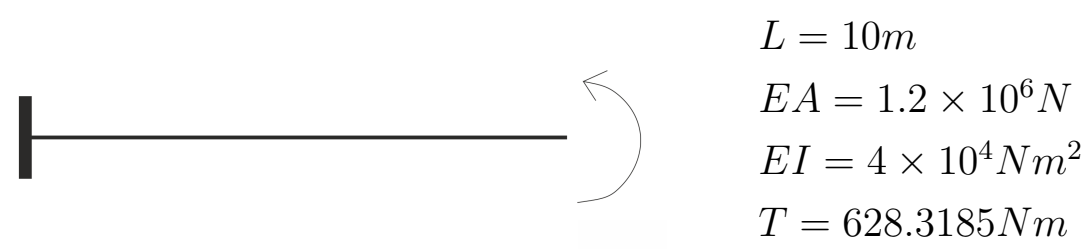

Figure 2.9: Geometry

For a twist of $\theta=2 \pi$, the required torque $T_{\max }=628.3185 \mathrm{Nm}$. The mesh consists of twenty equally spaced elements, and upon application of $T_{\max }$, a complete 
$2 \pi$ radians twist can be observed from Figure 2.10 which shows two different views of the beam.

\subsubsection{Roll-up of a Beam under end moment}

In this standard test problem, we present a Cantilever beam under concentrated moment at its free end. A full ring (tip rotation of $2 \pi$ of the Cantilever tip) is obtained upon application of the required moment, which from beam theory following
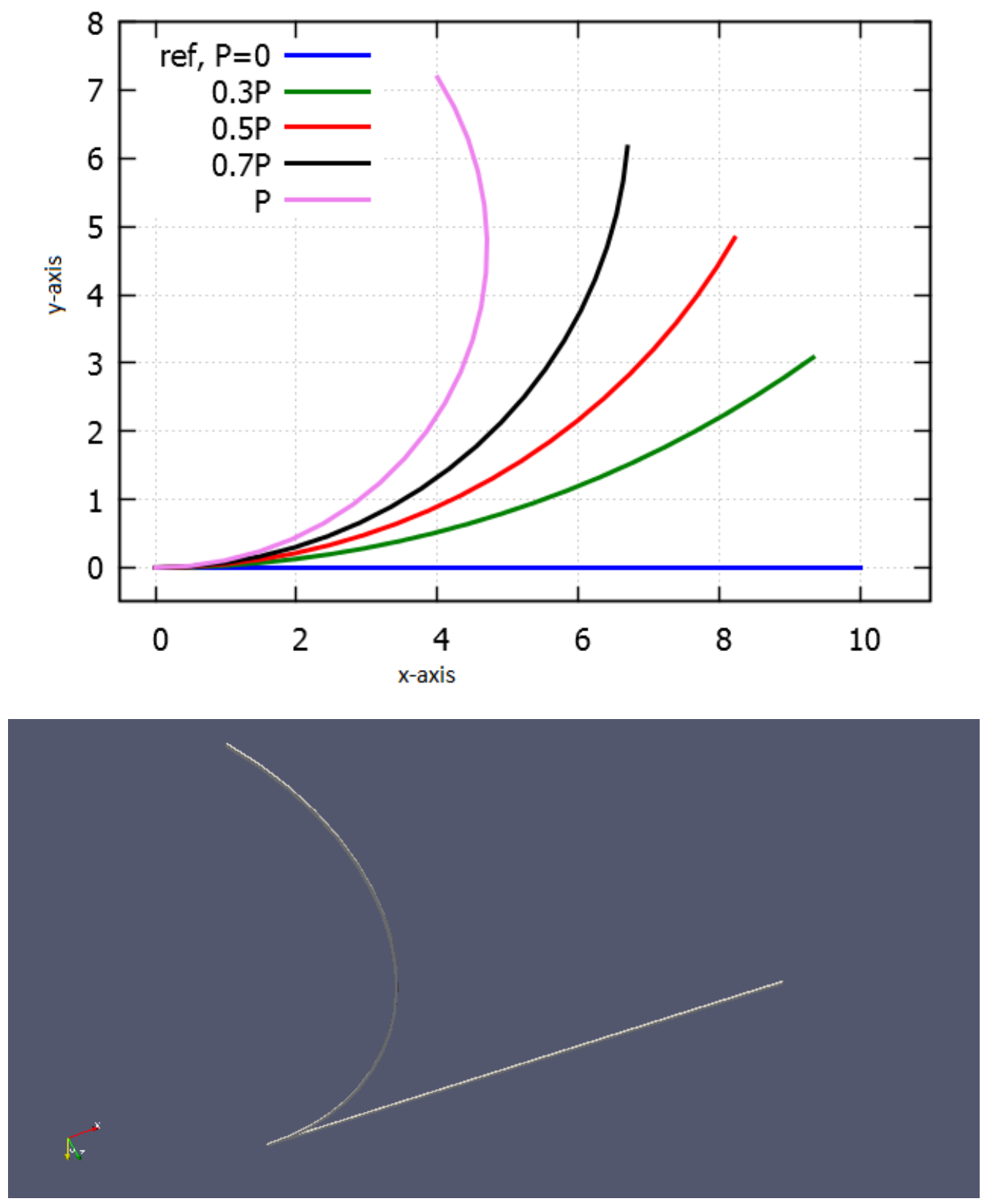

Figure 2.8: Large Cantilever Beam configurations under applied end moment 
from Equation 2.7 .3 is computed from

$$
M=\frac{2 \pi E I}{L}
$$

The properties of the Cantilever are chosen as $L=10 \mathrm{~m}$, and bending stiffness $E I=4 \times 10^{4} \mathrm{Nm}^{2}$. The full ring can be obtained in four load increments, and convergence is achieved in an average of four iterations per load increment as expected of the Newton method. Figure 2.12 shows the beam configuration at the load levels, and the displacement error (eccentricity between the clamped end and the tip end is $1.172 \times 10^{-15} \approx 0$.
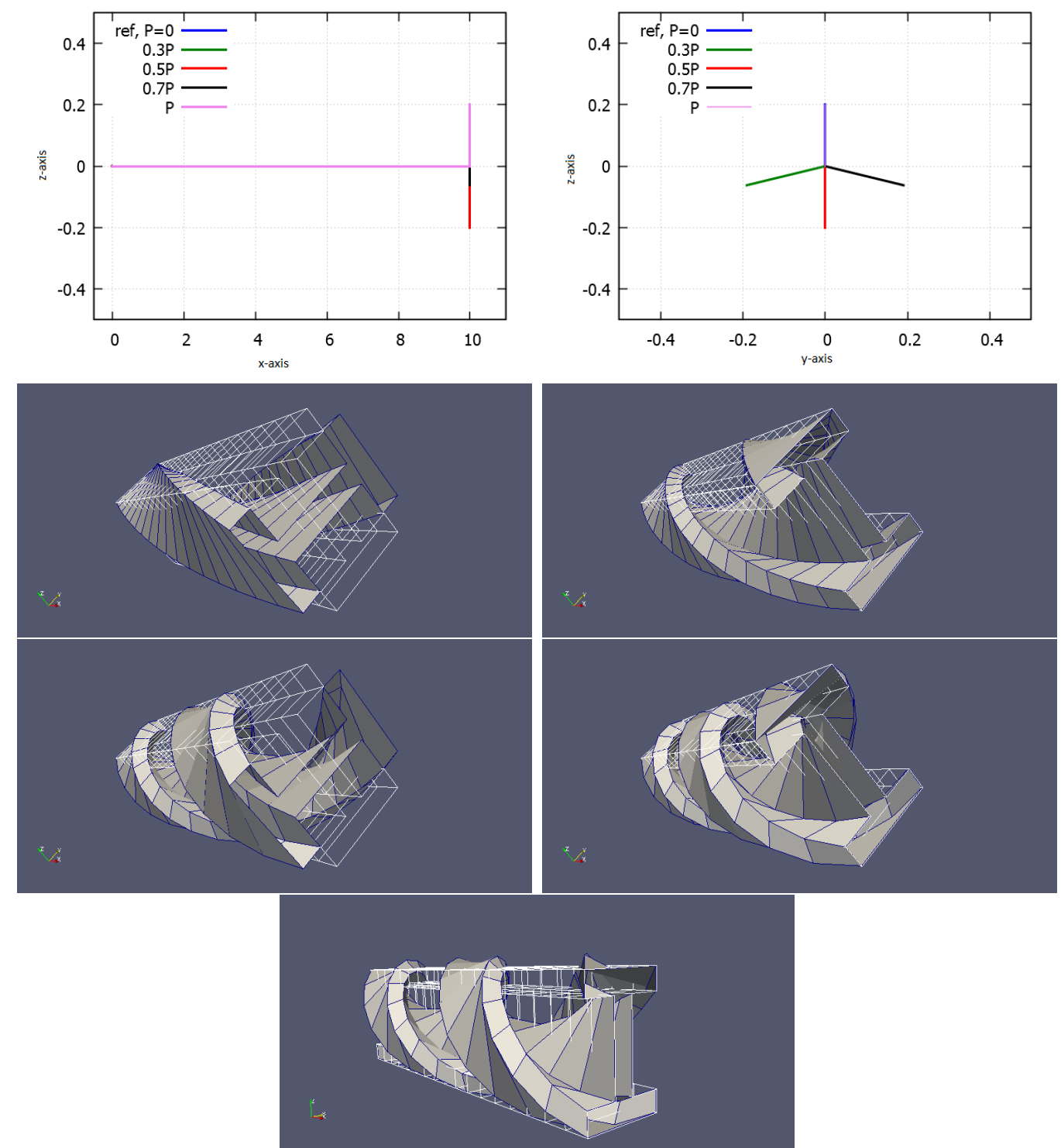

Figure 2.10: Large beam rotation (twist) under end torsion 


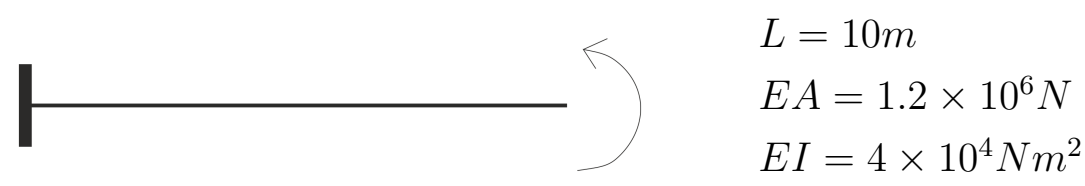

Figure 2.11: Geometry
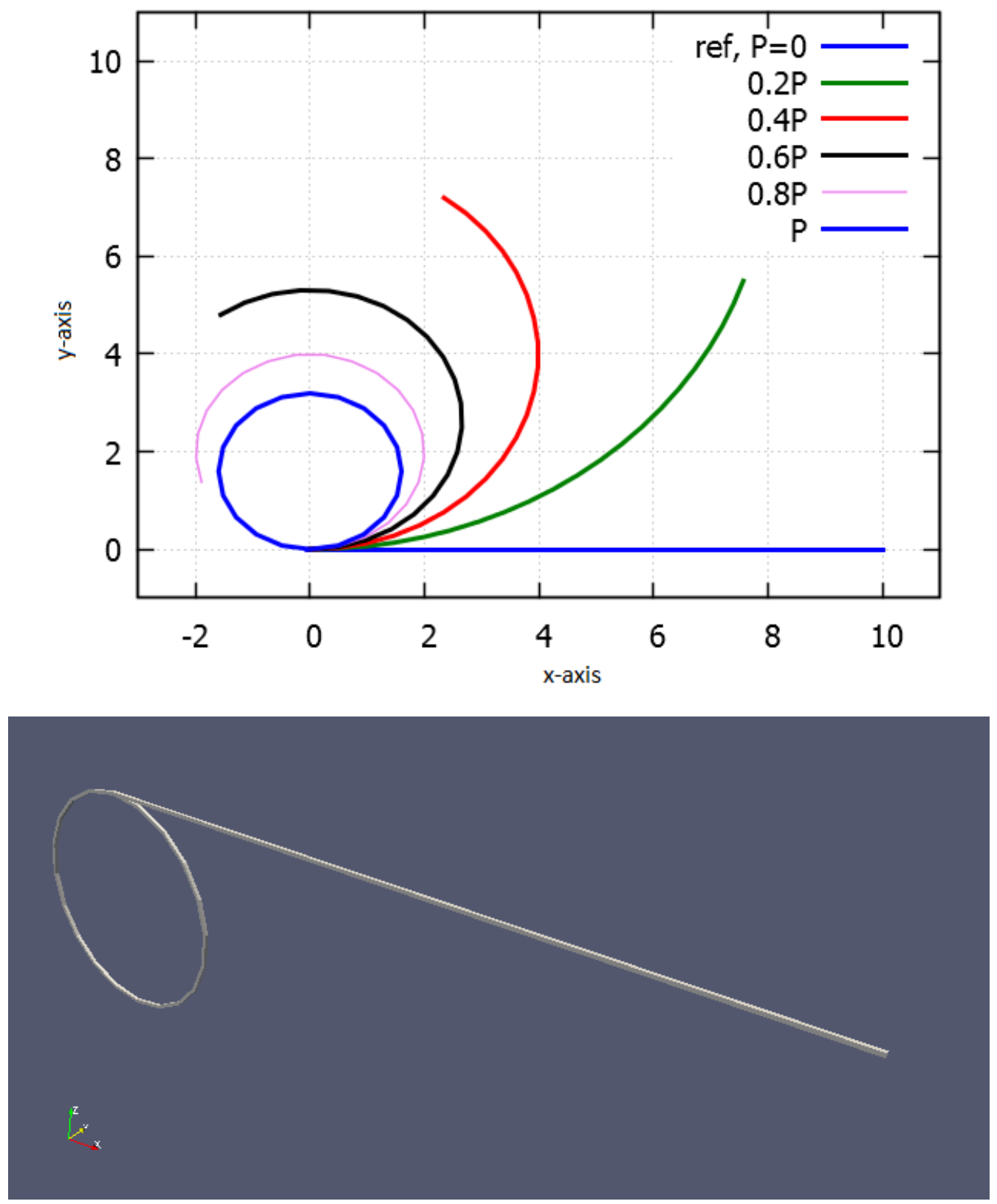

Figure 2.12: Roll-up of a beam under end moment

To reach the accuracy of $10^{9}$ which is the tolerance set, Table 2.4 shows the 
residuals for the last 2 load steps for the 20 and 50 load step cases respectively. As expected, the number of iterations required decreases as the number of load steps increases. This is expected of the solution procedure as the computational effort per step required gets smaller as the loading step increases. This is compared against the successful attempt to roll the beam with 4 load steps, but which required more iterations to converge on the solution.

\begin{tabular}{c|c|cc|cc}
\hline \multirow{2}{*}{ iteration } & 4 load steps & \multicolumn{2}{|c}{20 load steps } & \multicolumn{2}{c}{50 load steps } \\
& $t_{n}$ & $t_{n-1}$ & $t_{n}$ & $t_{n-1}$ & $t_{n}$ \\
\hline 1 & $6.28 \times 10^{3}$ & $1.25 \times 10^{3}$ & $1.25 \times 10^{3}$ & $5.027 \times 10^{2}$ & $5.03 \times 10^{2}$ \\
2 & $3.57 \times 10^{3}$ & $6.54 \times 10^{4}$ & $6.62 \times 10^{4}$ & $1.074 \times 10^{4}$ & $1.08 \times 10^{4}$ \\
3 & $3.51 \times 10^{3}$ & $1.07 \times 10^{3}$ & $1.08 \times 10^{3}$ & 5.227 & 5.24 \\
4 & $1.22 \times 10^{3}$ & $2.43 \times 10^{1}$ & $2.46 \times 10^{1}$ & $8.56 \times 10^{-} 2$ & $8.6 \times 10^{-} 2$ \\
5 & $1.54 \times 10^{3}$ & $1.06 \times 10^{-} 1$ & $1.08 \times 10^{-} 1$ & $3.91 \times 10^{-} 9$ & $6.86 \times 10^{-} 9$ \\
6 & $1.8867 \times 10^{2}$ & $2.6 \times 10^{-} 6$ & $2.66 \times 10^{-} 6$ & & \\
7 & $1.1299 \times 10^{2}$ & $7.25 \times 10^{-} 9$ & $4.1 e-9$ & & \\
8 & 1.101 & & & & \\
9 & $4.46 \times 10^{-} 3$ & & & & \\
10 & $5.22 \times 10^{-9}$ & & & & \\
\hline
\end{tabular}

Table 2.4: Convergence table for the 4, 20 and 50 load step cases

\subsubsection{Buckling of a narrow cantilever Beam}

We consider the buckling of a narrow cantilever beam subjected to an axial load. From elementary beam theory, the lowest critical or Euler buckling load for the first mode is given by

$$
P_{c r}=\frac{\pi^{2} E I}{L_{e}^{2}}
$$

where $L_{e}$ is the effective beam length depending on the beam end conditions, and for the cantilever beam is given as $L_{e}=2 L$.

Using a mesh of twenty 2-node linear elements for the beam with length $L=10 \mathrm{~m}$, cross-sectional area $A=0.4 \mathrm{~m}^{2}$, shear modulus $G=0.21 \times 10^{6} \mathrm{Nm}^{-2}$, bending stiffness $E I=4 \times 10^{4} \mathrm{Nm}^{2}$, and Young's modulus $E=3 \times 10^{6} \mathrm{Nm}^{-2}$, the Euler buckling load is computed to be $246.74 N$. 


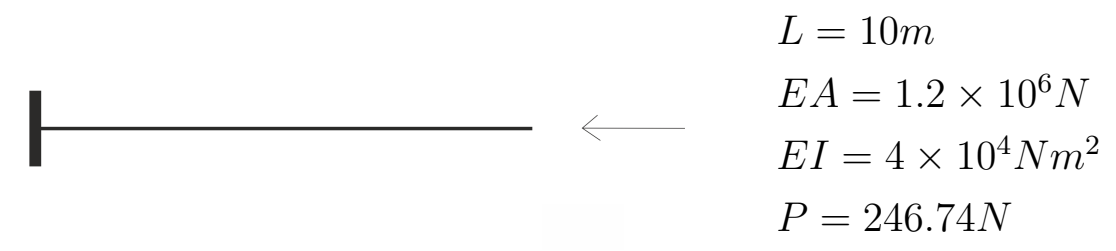

Figure 2.13: Geometry

The deformed beam configuration of the beam is presented in Figure 2.14. $P=$ $250 \mathrm{~N}$ was used for the simulation where the critical load was observed to be $247 \mathrm{~N}$, beyond which the snap-through behaviour was observed.

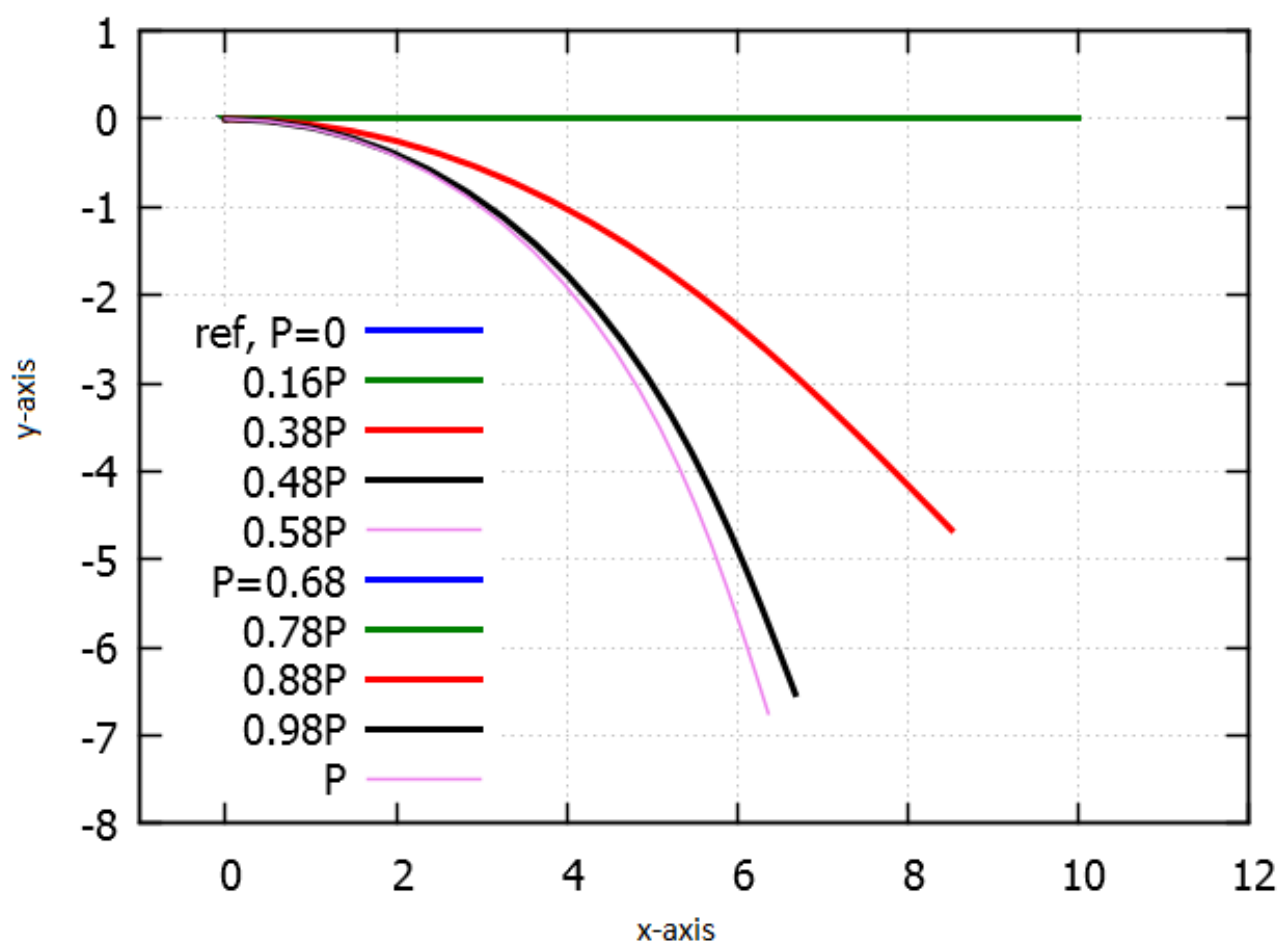

Figure 2.14: Buckling of a narrow Cantilever Beam

\subsubsection{Lee Frame}

In this example we investigate the buckling of a right-angle frame commonly known as the Lee frame. The Cantilever properties are $E A=4.32 \times 10^{4} \mathrm{KN}, G A=$ $2.16 \times 10^{4} \mathrm{KN}$, member length $L=1.2 \mathrm{~m}$, and $E I=1.44 \mathrm{KNm}^{2}$. A total of twenty 2-node linear elements are used to discretize the problem, and boundary conditions are applied at both ends to the translational degrees of freedom, allowing for rotation (pinned supports). The deformed shape of the beam at various load levels are shown 
in Figure 2.16. The limit load obtained which was $18.63 k N$ matched that obtained by other researchers (eg [8] with a limit load of $18.792 \mathrm{kN}$, and considering classical beam theory, $18.454 k N$ presented in the same paper). The residuals are shown in Table 2.5 to show quadratic convergence obtained from the simulation.

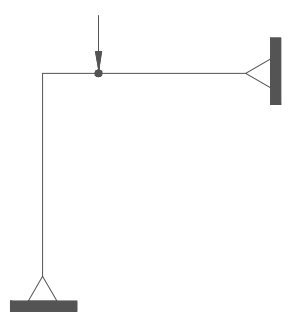

member length $L=1.2 \mathrm{~m}$

$E A=4.32 \times 10^{4} k N$

$E I=1.44 k N m^{2}$

reference load $P=1 k N$

Figure 2.15: Geometry

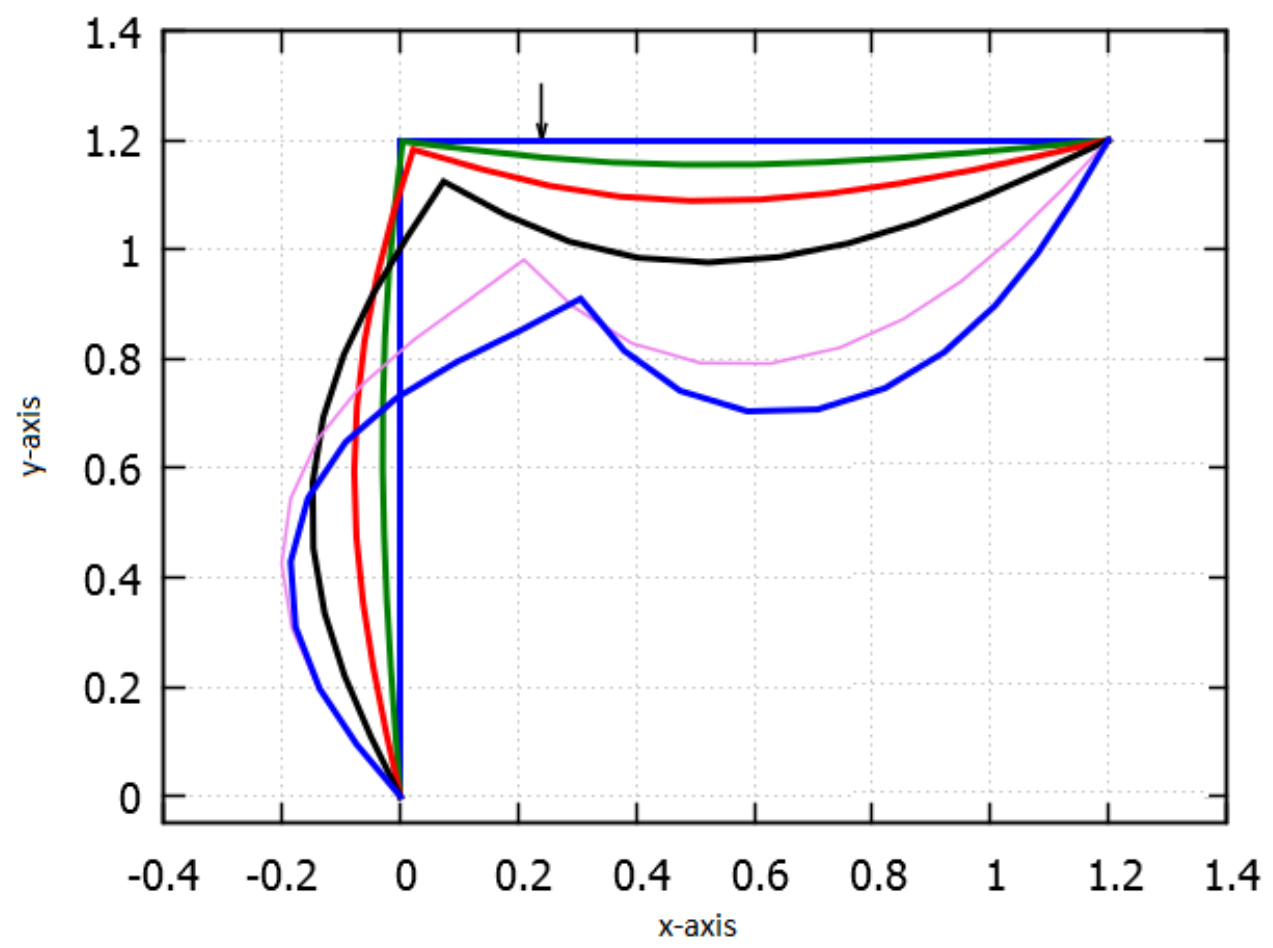

Figure 2.16: Large beam displacement under end moment 


\begin{tabular}{c|cc}
\hline \multirow{2}{*}{ iteration } & \multicolumn{2}{|c}{100 load steps } \\
\hline 1 & $t_{n-1}$ & $t_{n}$ \\
\hline 2 & $2 \times 10^{-} 1$ & $2 \times 10^{-} 1$ \\
3 & $1.196 \times 10^{1}$ & $1.296 \times 10^{1}$ \\
4 & $3.426 \times 10^{-} 3$ & $2.18 \times 10^{-} 3$ \\
5 & $2.397 \times 10^{-} 3$ & $5.77 \times 10^{-} 3$ \\
\hline
\end{tabular}

Table 2.5: Convergence table for 100 load steps

\subsubsection{Large displacement analysis of a $45^{\circ}$ Cantilever beam bend}

Studied by Bathe and Bolourchi, this example offers a three dimensional setting to test the element, experiencing all modes of deformation: shear, torsion, bending, and extension. It consists of a $45^{\circ}$ bend cantilever beam subjected to an end load normal to the plane.

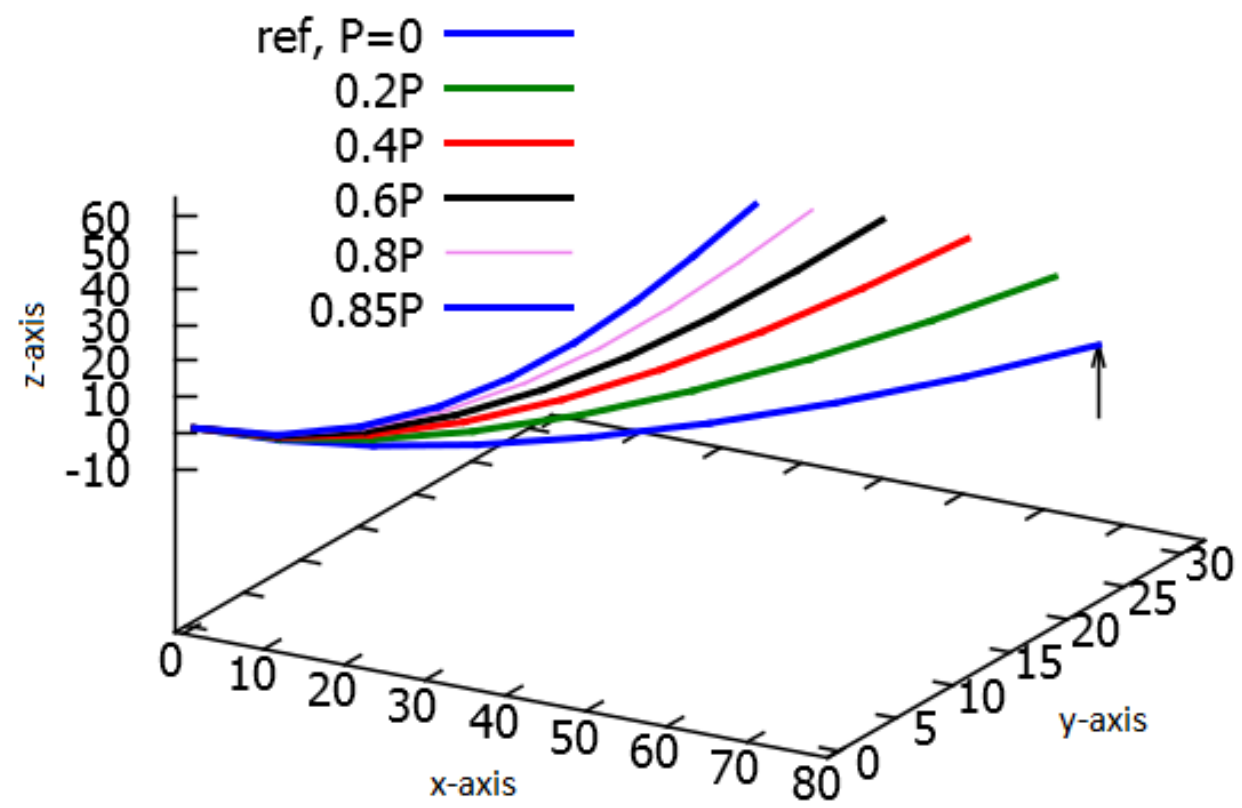

Figure 2.17: Deformed shape of the $45^{\circ}$ Cantilever bend

The reference configuration shown in Figure 2.17 is a $45^{\circ}$ circular arc with radius $r=100 \mathrm{in}$ and unit cross sectional area. Material properties are: Young's modulus 


\begin{tabular}{c|ccc} 
& $U_{x}$ & $U_{y}$ & $U_{z}$ \\
\hline Bathe and Bolourchi [5] & -13.39 & -23.51 & 53.4 \\
Crisfield [17] & -13.68 & -23.87 & 53.71 \\
Simo and Vu-Quoc [53] & -13.5 & -23.48 & 53.37 \\
Cesarek and Zupan [14] & -13.48 & -23.48 & 53.27 \\
Present & -13.67 & -23.71 & 53.5 \\
\hline
\end{tabular}

Table 2.6: Comparison of tip displacements for the $45^{\circ}$ bend

$E=10^{7} \mathrm{psi}$, and Poisson's ratio $\nu=0$. Under an applied load at the free end $P=600$, and the results agree with those available in literature. The deflection of the free end of the beam is given in Table 2.6. Applying the load in 10 increments, the convergence table is shown in Table 2.7 below

\begin{tabular}{c|cc}
\hline \multirow{2}{*}{ iteration } & \multicolumn{2}{|c}{ 10 load steps } \\
\hline 1 & $t_{n-1}$ & $t_{n}$ \\
2 & $6 \times 10^{1}$ & $6 \times 10^{1}$ \\
3 & $6.328 \times 10^{4}$ & $5.201 \times 10^{4}$ \\
4 & $1.084 \times 10^{1}$ & $1.606 \times 10^{1}$ \\
5 & $6.35 \times 10^{-} 5$ & $7.216 \times 10^{1}$ \\
6 & $7.86 \times 10^{-} 9$ & $7.019 \times 10^{-} 5$ \\
\hline
\end{tabular}

Table 2.7: Convergence table for 10 load steps

\subsection{Conclusion}

Following the work by Ibrahimbegovic [31] where only 3 parameters were used to explore vector-like parametrization of three dimensional finite rotations, the Rodriguez formula is used to construct the rotation tensor which maps the base vectors of the reference frame onto the current frame. The motion of the beam is completely defined, and the axial, shear, and bending strains follow. Linearization of the equations required by the newton method are performed, and the tangent operator obtained for use in the solution of the finite element equation.

A number of simulations have been run to study the accuracy, convergence, and capabilities of the finite element. The numerical results show that the finite element is capable of handling finite deformations and rotations, which will be required in subsequent chapters of this work. Excellent agreement with results available in literature, including those with available analytical solutions as well as those directly compared against result from other researchers shows high accuracy of the element, 
and expected behaviour of the various systems is observed. The convergence tables presented, demonstrate quadratic convergence which is expected from the NewtonRaphson solution procedure, as a result of accurate linearization of the finite element equations.
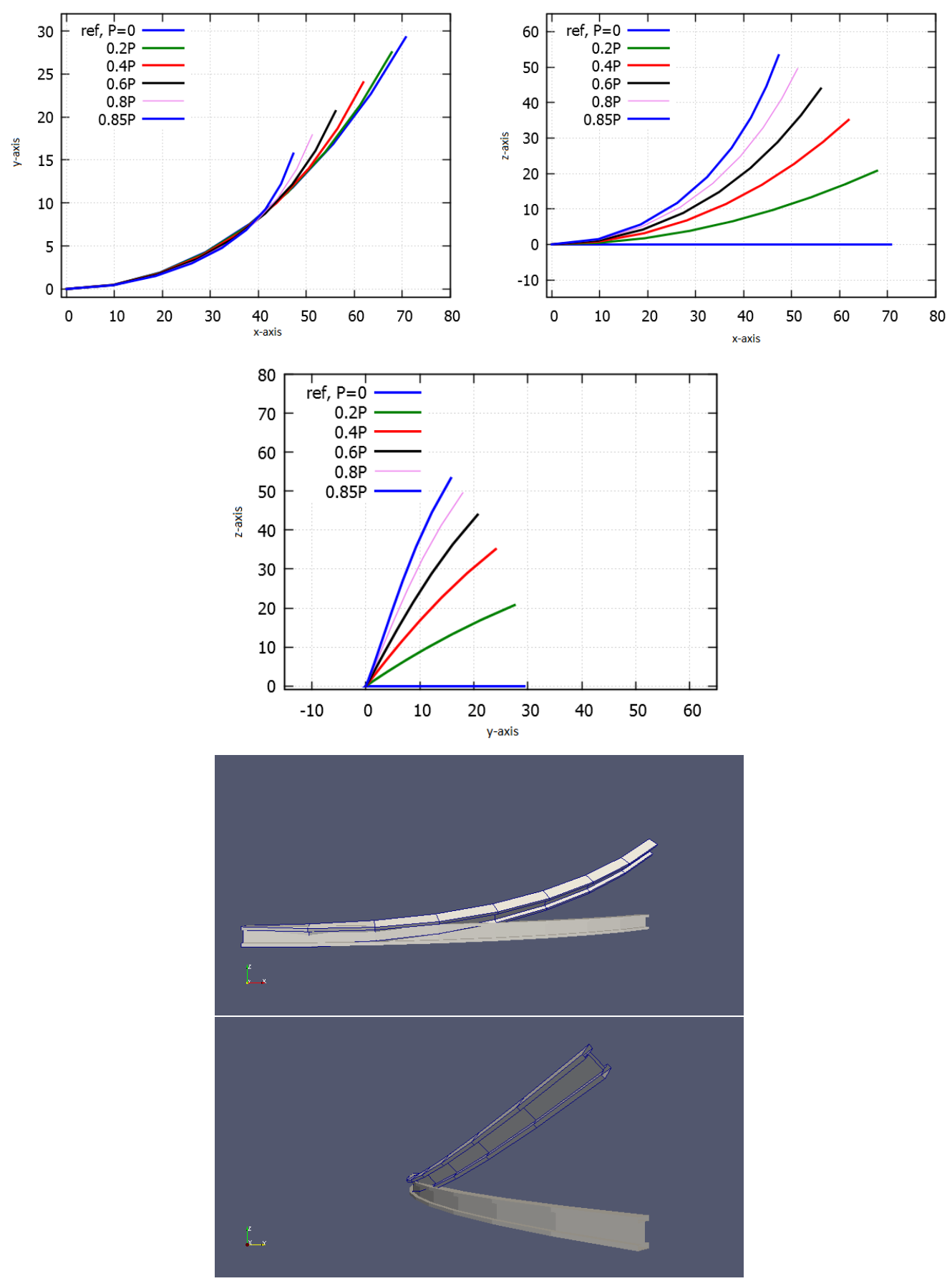

Figure 2.18: Large displacement analysis of a $45^{\circ}$ Cantilever beam bend 


\section{Chapter 3}

\section{Vibration of beams}

Here, we present a brief chapter to show the ability of our approach to solve dynamic problems. The equation, and time stepping scheme used here and in subsequent sections of this work is presented, as well as an example to show some results from simulations.

\subsection{Introduction}

In addition to the components that contribute to the internal forces and tangent stiffness matrices of a nonlinear beam element, the development of nonlinear dynamic beam elements require treatment of finite rotations, and application of a appropriate time-stepping scheme. Various time integration schemes used in literature include, but is not limited to Runge-Kutta methods, Wilson schemes, with the Newmark methods being very popular. The finite rotations in nonlinear dynamic analysis can be parametrized in several ways. Spin variables, both spatial and material, have been adopted in various works in literature. The 'additive' advantage that the use of rotational variables affords, makes the use of the rotational vectors an attractive alternative. The use of the total rotational vector restricts the angle of rotation to $2 \pi$, which has led to alternatives including the incremental rotation vector and a switching procedure to avoid the limitation. Dynamics of beams can also be formulated using rotational quarternions, as well as the conformal rotation vector. The Newmark scheme is widely used for time integration in their various adjustments depending on the requirements of the choice of parametrization.

One of the earlier key work in this field was by Simo [56], where spatial spin variables were used to parametrize rotations, and the Newmark equations were written using the material incremental rotational vector, the material angular velocity and the material acceleration. This approach was also adopted by Crisfield et al. [18], 
Jelenic and Crisfield [35] [36], and Hsiao et al. [27]. Different types of implicit time integration algorithms were discussed, and the beam element was formulated using the co-rotational technique in [18]. Hsiao [27] in their work, presented a co-rotational formulation for dynamics of spatial Euler beams with large rotations. The nonlinear equations of motion were solved using an incremental-iterative method based on the Newmark direct integration method, and the Newton-Raphson method. Cardona and Geradin [13] examined a couple of formulations, for the nonlinear dynamic beam element, also making use of the incremental rotation vector to overcome the limitation of the angle of rotation. Ibrahimbegovic [30] [32] also adopted the incremental rotation vector, to which, reformulated in their spatial forms, the standard Newmark algorithm was applied. Avoiding the limitations of rotation angles by employing a switching procedure was employed by Makinen [43], and Iura [33] adopted the approach of using the material form of the incremental rotation vector in the Newmark equations. Betsch and Steinmann [9], extending the work in [8] for dynamics, linearly interpolated the nodal rotations parametrized with the rotation matrix, requiring nine rotational variables at each node hence not ensuring the orthonormality of the interpolated rotation matrix. Geradin [24] described the conformal rotation vector, a technique used to parametrize finite rotations using only 3 independent parameters in his work. Battini [6] parametrized large rotations in corotational beam elements presenting several alternatives, and a comparative study of new and established formulations in the corotational context can be found in [39]. A recent work based on the Newmark scheme and a quarternion description, a dynamic beam formulation can be found in 63 , and a consistent 3D corotational beam element for nonlinear dynamic analysis presented in [40]. Additional information on the subject matter can be found in [54] [55] [52].

\subsection{Equation of motion}

In the absence of fluid flow, our equation of motion is

$$
E I \frac{\partial^{4} y}{\partial x^{4}}+m \frac{\partial^{2} y}{\partial t^{2}}=0
$$

where $E I$ is the bending stiffness of the pipe, $y$ is the pipe deflection, and $m$ is the pipe mass per unit length.

The first term in Equation 3.2.1 relates to the beams, for which we use the geometrically exact formulations in $2 \mathrm{D}$ and $3 \mathrm{D}$ to which the inertia term is added for simulation of dynamics. For the additional inertia term, using the 2-node linear 
finite element, we have

$$
\int_{0}^{l}(m)(s) \ddot{y}(s, t) \delta y(s) d s
$$

Simplifying, we obtain the consistent mass matrix as

$$
\boldsymbol{M}^{e}=\int_{0}^{l^{e}}(m) N_{1} N_{2} d s
$$

which yields

$$
\boldsymbol{M}^{e}=\frac{m l}{6}\left[\begin{array}{llllllllllll}
2 & 0 & 0 & 0 & 0 & 0 & 1 & 0 & 0 & 0 & 0 & 0 \\
0 & 2 & 0 & 0 & 0 & 0 & 0 & 1 & 0 & 0 & 0 & 0 \\
0 & 0 & 2 & 0 & 0 & 0 & 0 & 0 & 1 & 0 & 0 & 0 \\
0 & 0 & 0 & 0 & 0 & 0 & 0 & 0 & 0 & 0 & 0 & 0 \\
0 & 0 & 0 & 0 & 0 & 0 & 0 & 0 & 0 & 0 & 0 & 0 \\
0 & 0 & 0 & 0 & 0 & 0 & 0 & 0 & 0 & 0 & 0 & 0 \\
1 & 0 & 0 & 0 & 0 & 0 & 2 & 0 & 0 & 0 & 0 & 0 \\
0 & 1 & 0 & 0 & 0 & 0 & 0 & 2 & 0 & 0 & 0 & 0 \\
0 & 0 & 1 & 0 & 0 & 0 & 0 & 0 & 2 & 0 & 0 & 0 \\
0 & 0 & 0 & 0 & 0 & 0 & 0 & 0 & 0 & 0 & 0 & 0 \\
0 & 0 & 0 & 0 & 0 & 0 & 0 & 0 & 0 & 0 & 0 & 0 \\
0 & 0 & 0 & 0 & 0 & 0 & 0 & 0 & 0 & 0 & 0 & 0
\end{array}\right]
$$

The time stepping scheme used is shown below, and the contributions from the various terms above are put together to obtain the global element force vector and the global element tangent operator. The assembly of elements results in the global system of equations which is solved for the required degrees of freedom at the nodes.

\subsection{Finite Element Implementation}

Similar to the previous chapter, for the finite element approximation, the pipe is divided into elements and shape functions are introduced to approximate the pipe deflection $y$. This is given by

$$
\left\{\begin{array}{l}
\boldsymbol{y}^{e} \\
\boldsymbol{\theta}^{e}
\end{array}\right\}=\sum_{a=1}^{n}\left[\begin{array}{cc}
N_{a} & 0 \\
0 & N_{a}
\end{array}\right]\left\{\begin{array}{l}
\boldsymbol{u}_{a} \\
\boldsymbol{\theta}_{a}
\end{array}\right\}
$$

where $n$ is the number of element nodes. For a 2-noded linear element, we have that 


$$
\begin{aligned}
N_{1} & =\frac{1-\xi}{2} \\
N_{2} & =\frac{1+\xi}{2} \\
\text { and } \quad d x & =\frac{l_{e}}{2} d \xi
\end{aligned}
$$
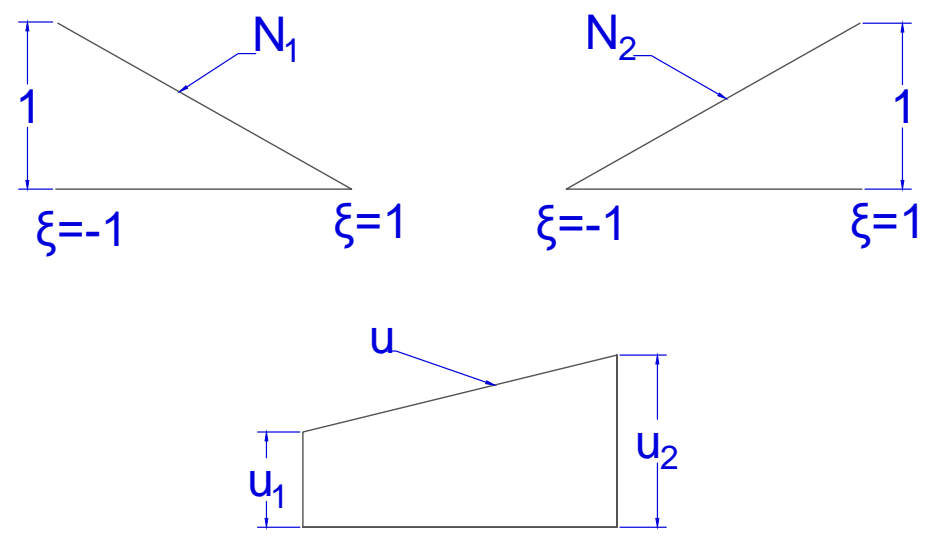

Figure 3.1: 2 node linear shape functions

The finite element solution of this non-linear problem requires the use of the Newton-Raphson iterative process whose iterative process uses a solution estimate $\boldsymbol{x}_{k}$ at iteration $k$ to obtain a new value $\boldsymbol{x}_{k+1}=\boldsymbol{x}_{k}+\boldsymbol{u}$ in terms of an increment $\boldsymbol{u}$ by establishing the linear approximation

$$
\boldsymbol{R}\left(\boldsymbol{x}_{k+1}\right) \approx \boldsymbol{R}\left(\boldsymbol{x}_{k}\right)+D \boldsymbol{R}\left(\boldsymbol{x}_{k}\right)[\boldsymbol{u}]=\mathbf{0}
$$

a linear set of equations is solved at each Newton-Raphson iteration as

$$
\boldsymbol{K}\left(\boldsymbol{x}_{k}\right) \boldsymbol{u}=-\boldsymbol{R}\left(\boldsymbol{x}_{k}\right) ; \quad \boldsymbol{x}_{k+1}=\boldsymbol{x}_{k}+\boldsymbol{u}
$$

The quantities that go into the solution are presented in Equation 3.4 .4 and Equation 3.4.5, assembled from the element contributions into the global system of 
equations.

\subsection{Time stepping scheme}

The generalised mid-point rule is used for the discretisation of time, where we solve for the deflection $y_{n+1}$ at time $t_{n+1}$ using deflection, velocity and acceleration at time $t_{n+\gamma}$, with $\frac{1}{2} \leq \gamma \leq 1$.

The choice of the parameter $\gamma$ affects the accuracy as well as the amount of numerical damping of the solution. With $\gamma=0$ we have the forward Euler method which is fully explicit. Using $\gamma=1$ results in a first order accurate solution with strong numerical damping known as the backward Euler method. The trapezoidal(midpoint) rule which is second order accurate with no numerical damping is obtained when we choose $\gamma=\frac{1}{2}$.

We compute the deflection, velocity and acceleration at time $t_{n+\gamma}$ with

$$
\begin{aligned}
y_{n+\gamma} & =\gamma y_{n+1}+(1-\gamma) y_{n} \\
\dot{y}_{n+\gamma} & =\gamma \dot{y}_{n+1}+(1-\gamma) \dot{y}_{n}=\frac{y_{n+1}-y_{n}}{\Delta t} \\
\ddot{y}_{n+\gamma} & =\frac{\dot{y}_{n+1}-\dot{y}_{n}}{\Delta t}
\end{aligned}
$$

Rearranging Equation 3.4.1 for $\ddot{y}_{n+\gamma}$ we have

$$
\begin{aligned}
& \dot{y}_{n+1}=\frac{y_{n+1}-y_{n}}{\gamma \Delta t}-\frac{1-\gamma}{\gamma} \dot{y}_{n} \\
& \ddot{y}_{n+\gamma}=\frac{y_{n+1}-y_{n}}{\gamma \Delta t^{2}}-\frac{1}{\gamma \Delta t} \dot{y}_{n}
\end{aligned}
$$

With Equations 3.4.1, 3.4.2, and the equation of motion (Equation 3.2.1), we obtain the element force vector and stiffness matrices, $\boldsymbol{f}^{e}$. The consistent form of the mass matrix is used, and the explicit expressions for the element stiffness matrix are given in their respective chapters (2D and $3 \mathrm{D})$

$$
\boldsymbol{f}^{e}=\boldsymbol{K}^{e} \boldsymbol{y}_{n+\gamma}^{e}+\boldsymbol{M}^{e} \ddot{\boldsymbol{y}}_{n+\gamma}^{e}
$$


Rearranging Equation 3.4.3, we have

$$
\begin{aligned}
\boldsymbol{f}^{e}= & \left(\boldsymbol{K}^{e} \gamma+\boldsymbol{M}^{e} \frac{1}{\gamma \Delta t^{2}}\right) \boldsymbol{y}_{n+1}^{e} \\
& +\left(\boldsymbol{K}^{e}(1-\gamma)-\boldsymbol{M}^{e} \frac{1}{\gamma \Delta t^{2}}\right) \boldsymbol{y}_{n}^{e} \\
& -\boldsymbol{M}^{e} \frac{1}{\gamma \Delta t} \dot{\boldsymbol{y}}_{n}^{e}
\end{aligned}
$$

The element tangent operator follows directly from Equation 3.4 .4 as

$$
\boldsymbol{K}^{e} \gamma+\boldsymbol{M}^{e} \frac{1}{\gamma \Delta t^{2}}
$$

In the usual manner, the Global system $\boldsymbol{A} \boldsymbol{y}_{n+1}=\boldsymbol{b}$ is assembled from the elements and solved for the required degrees of freedom (deflection and rotation) at the nodes.

\subsection{Numerical example}

\subsubsection{Lee's frame}

A Lee's frame undergoing forced vibration is presented for our test of the 2D nonlinear finite element. The example is taken from [38], and the frame is shown in Figure 3.2 with $L=2.4 m, a=0.2 m$, and $e=0.3 m$. Young's modulus $E=210 G P a$, and mass per unit volume, $\rho=7850 \mathrm{~kg} / \mathrm{m}^{3}$. A constant load of $P=4.1 M N$ is applied as shown in the figure, and the horizontal and vertical displacements over time, recorded and compared against the result presented in [38].

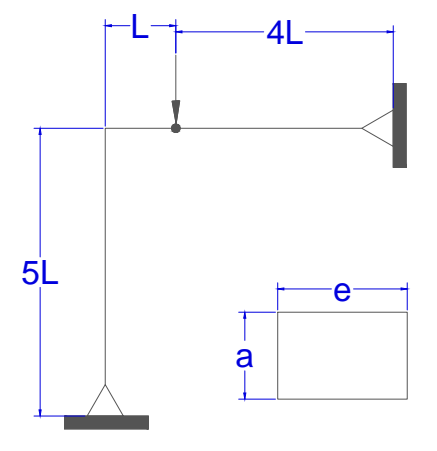

$$
\begin{aligned}
& a=0.2 \mathrm{~m} \\
& e=0.3 \mathrm{~m} \\
& L=2.4 \mathrm{~m} \\
& E=210 \mathrm{GPa} \\
& \rho=7850 \mathrm{~kg} / \mathrm{m}^{3} \\
& P=4.1 \mathrm{MN} \\
& \Delta t=2.5^{-3} \mathrm{~s}
\end{aligned}
$$

Figure 3.2: Geometry

50 2-node linear finite elements were used in this simulations and the results are 
presented in Figures 3.3 and 3.4 compared against the result obtained from [38]. From the time histories, it can be observed that the result of our simulation is in good agreement with the reference solution. In his work ([38]), compared results using various mass matrices, but his reference is what our results are compared with.

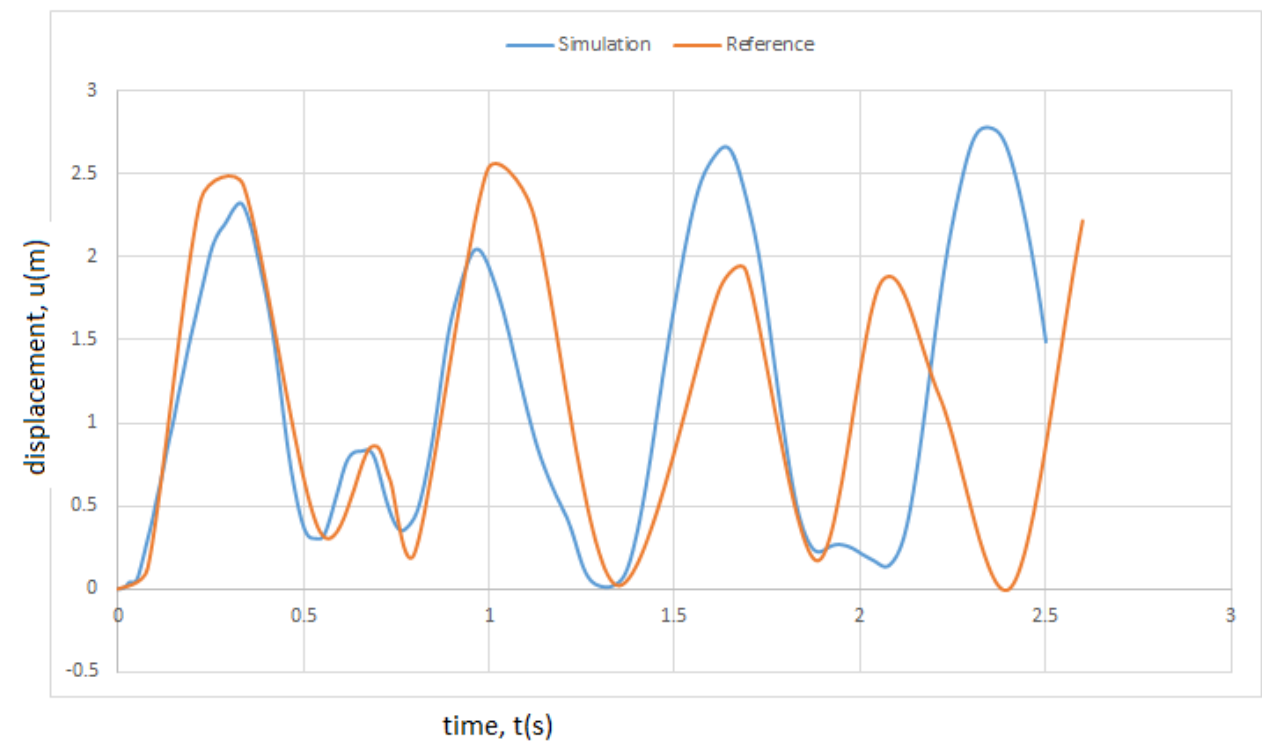

Figure 3.3: horizontal displacement

The table of residuals is presented in Table 3.1, for the last 2 time steps of the simulation and show quadratic convergence, as expected of the Newton-Raphson solution procedure.

\begin{tabular}{c|cc}
\hline iteration & $t_{n-1}$ & $t_{n}$ \\
\hline 1 & $1.8 \times 10^{3}$ & $1.766 \times 10^{3}$ \\
2 & $6.21 \times 10^{5}$ & $4.12 \times 10^{5}$ \\
3 & $3.08 \times 10^{3}$ & $1.88 \times 10^{3}$ \\
4 & $1.23 \times 10^{1}$ & $5.61 \times 10^{1}$ \\
5 & $0.77 \times 10^{-} 2$ & $1.95 \times 10^{-} 2$ \\
6 & $4.31 \times 10^{-} 7$ & $7.019 \times 10^{-} 6$ \\
\hline
\end{tabular}

Table 3.1: Table of residuals for the last 2 time steps of the simulation

\subsection{Conclusion}

In this section, the nonlinear dynamic beam element was presented and compared against available examples. The time stepping scheme adopted for the discretization of time is the generalized mid-point rule, with the parametrization of finite rotations 


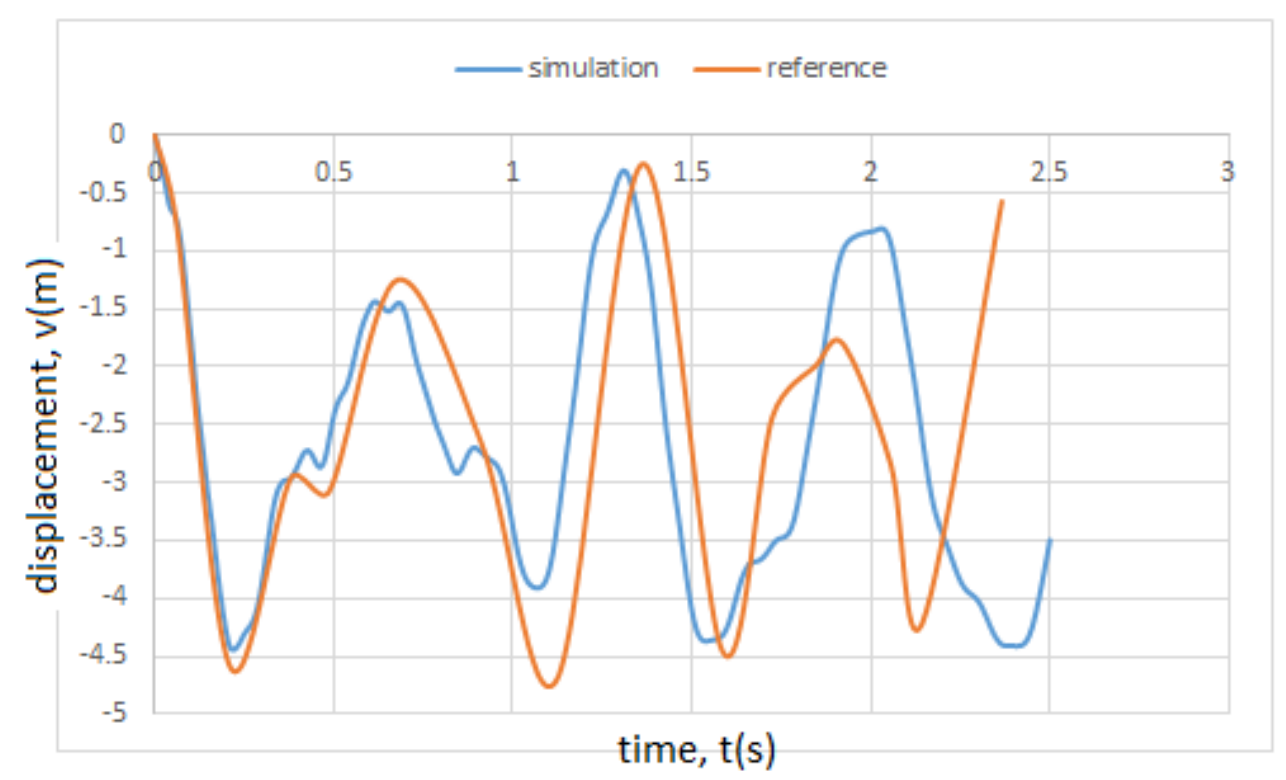

Figure 3.4: vertical displacement

by the orthogonal tensor using the total rotation vector. While the fundamental deficiency of this parametrization is the presence of singularity for for total rotation close to multiples of $2 \pi$, it is sufficient for our applications, and an appropriate choice of time step size ensures convergence, accuracy, and stability of the scheme.

The quadratic rate of convergence at each step demonstrated the accuracy of the computed tangent operator, as this is necessary for an efficient performance of the scheme. After confirming the accuracy of the nonlinear dynamics results, the next sections of this work proceed to investigate pipes conveying fluid, in two and three dimensions, in both linear and nonlinear regimes. 


\section{Chapter 4}

\section{Pipes Conveying fluid: 2D Linear dynamics}

\subsection{Introduction}

With the introduction of fluid flow through the pipe, this chapter builds upon the previous work by presenting a Linear $2 \mathrm{D}$ solution of the equation of motion to be derived in the subsequent section. This will serve as a starting point from which the nonlinear 2D solution, and the 3D elements will follow. The equation of motion is presented, the time stepping scheme and finite element solution follow, and some examples are presented to show the accuracy of the solution.

\subsection{Equations of motion}

Consider a pipe span of length $L$, with modulus of elasticity $E$, area moment of inertia $I$, and transverse deflection $y(x, t)$ from equilibrium as a result of internal fluid flow shown in Figure 4.1 [10]. The fluid flows at constant velocity $v$ through the internal cross-sectional area $A$, with density $\rho$ and pressure $p$.

Decomposing the system into fluid and solid subsystems in Figure 4.2 and Figure 4.3 [45] respectively for clarity, and formulating equilibrium, results in the equation of motion for transverse vibration of a straight, fluid conveying pipe.

\section{Fluid subsystem:}

As the pipe deflects due to fluid flow, the shear stress of fluid friction opposes the fluid pressure gradient along the pipe length. As a result of the changing curvature, the fluid also accelerates and is opposed by the vertical component of fluid pressure, and the pressure force from the pipe walls. 


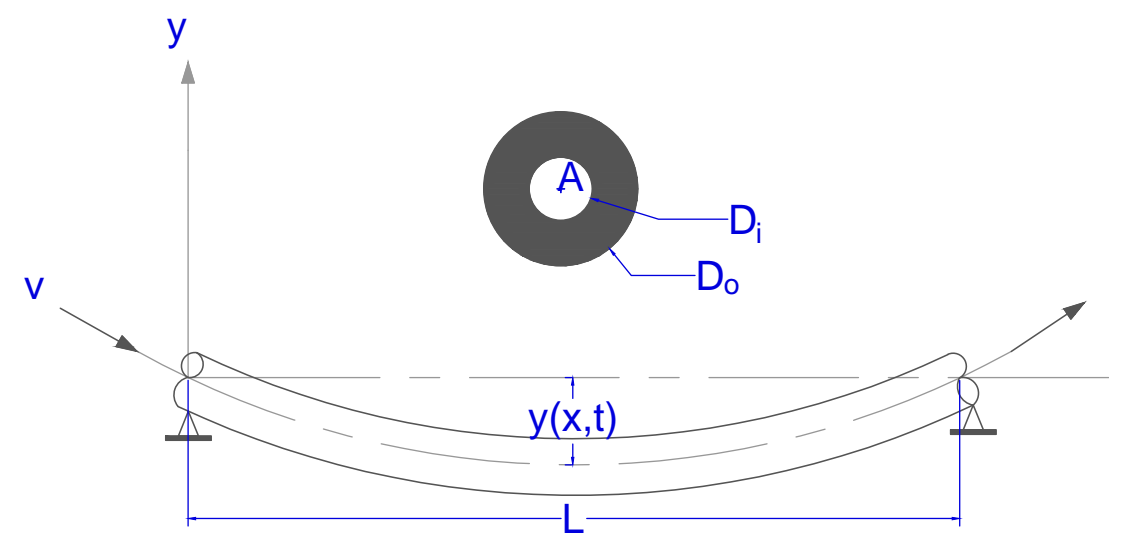

Figure 4.1: Pipe conveying fluid at constant velocity $v$

In $x$-direction, we have

$$
A \frac{\partial p}{\partial x}+\tau s=0
$$

In $y$-direction,

$$
F=A p \frac{\partial^{2} y}{\partial x^{2}}+\rho A\left(\frac{\partial}{\partial t}+v \frac{\partial}{\partial x}\right)^{2} y
$$

where $s$ is the internal cross-section circumference, $\tau$ is the wall shear stress, and $F$ is the force that the fluid exerts on the solid.

\section{Solid subsystem:}

In $x$-direction,

$$
\tau s+\frac{\partial T}{\partial x}-Q \frac{\partial^{2} y}{\partial x^{2}}=0
$$

In $y$-direction,

$$
F+m \frac{\partial^{2} y}{\partial t^{2}}-\frac{\partial Q}{\partial x}-T \frac{\partial^{2} y}{\partial x^{2}}=0
$$

For moments,

$$
\frac{\partial M}{\partial x}+Q=0
$$




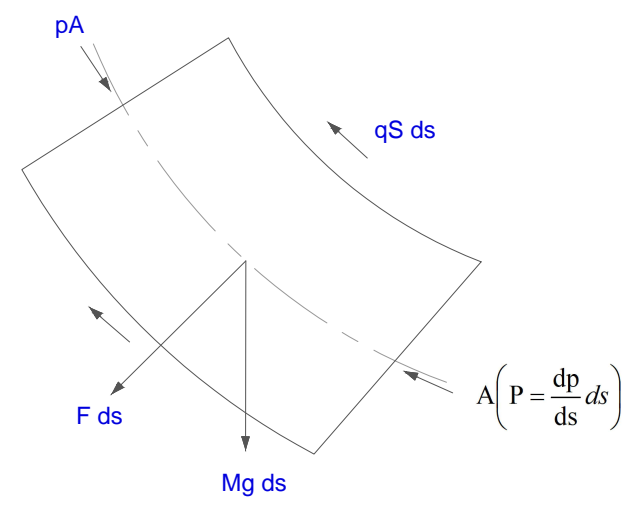

Figure 4.2: Forces acting on the fluid element

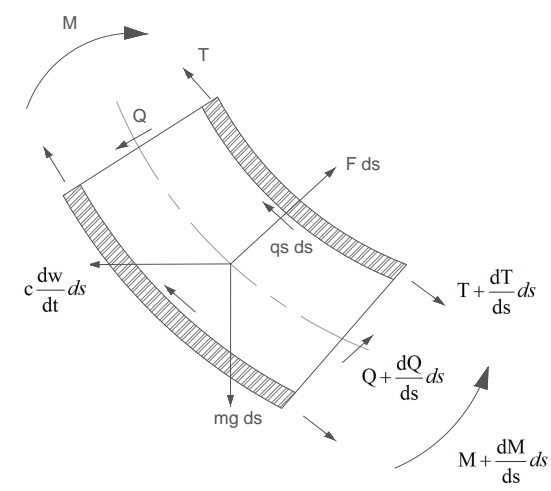

Figure 4.3: Forces acting on the solid element

where $m$ is the mass of the pipe per unit length, $T$ is the axial force in the pipe, $Q$ and $M$ are the internal shear force and bending moment in the pipe.

Eliminating the wall shear stress from Equations 4.2.1 and 4.2.3, we have

$$
A \frac{\partial p}{\partial x}-\frac{\partial T}{\partial x}+Q \frac{\partial^{2} y}{\partial x^{2}}=0
$$

with $M=E I \frac{\partial^{2} y}{\partial x^{2}}$ and Equation 4.2 .5 , the shear force $Q$ in the pipe

$$
Q=-E I \frac{\partial^{3} y}{\partial x^{3}}
$$

The third term in Equation 4.2.6 is quadratic and is neglected for linear analysis 
to give

$$
\frac{\partial}{\partial x}(p A-T)=0
$$

This implies that $p A-T$ is constant along the pipe span and is independent of position. Assuming that the fluid pressure is equal to the ambient pressure, and the tension in the pipe at the outflow boundary is zero, we have $p=T=0$ at $x=L$ hence

$$
p A-T=0 \quad \forall x \in(0, L)
$$

From Equations 4.2.2, 4.2.4, 4.2.7 and 4.2.9, we obtain the equation of motion as

$$
E I \frac{\partial^{4} y}{\partial x^{4}}+\rho A v^{2} \frac{\partial^{2} y}{\partial x^{2}}+2 \rho A v \frac{\partial^{2} y}{\partial x \partial t}+(m+\rho A) \frac{\partial^{2} y}{\partial t^{2}}
$$

The first term is the pipe stiffness, the second is the centrifugal force term, the third is the Coriolis force term which leads to flutter-like instability, and the last term is the inertia term.

\subsection{Finite Element Solution}

Applying the principle of virtual work for a finite element simulation, we rewrite Equation 4.2.10 in the weak form by integration by parts, reducing the fourth order derivative. Using $(\bullet)^{\prime}=\frac{\partial(\bullet)}{\partial x}$, we have for the bending stiffness term

$$
\begin{array}{r}
\int_{0}^{l} \delta y E I y^{\prime \prime \prime \prime} d x=0 \\
{\left[\delta y E I y^{\prime \prime \prime}\right]_{0}^{l}-\int_{0}^{l} \delta y^{\prime} E I y^{\prime \prime \prime} d x=0} \\
{\left[\delta y E I y^{\prime \prime \prime}\right]_{0}^{l}-\left[\delta y^{\prime} E I y^{\prime \prime}\right]_{0}^{l}+\int_{0}^{l} \delta y^{\prime \prime} E I y^{\prime \prime} d x=0}
\end{array}
$$

The first 2 terms of Equation 4.3.1 vanish for standard boundary conditions and the deflections $y(x, t)$ and admissible virtual deflections $\delta y(x)$ are constructed using the same shape functions.

Dividing the beam/pipe into several elements, each node has 2 degrees of freedom representing the deflection and slope or rotation at each node. we have for the 
displacement vector

$$
\boldsymbol{y}=\left[y_{1}, y_{1}^{\prime}, y_{2}, y_{2}^{\prime}\right]^{T}
$$

Satisfying continuity requirements, Hermite shape functions of cubic order are defined as shown in Figure 4.4 [15].
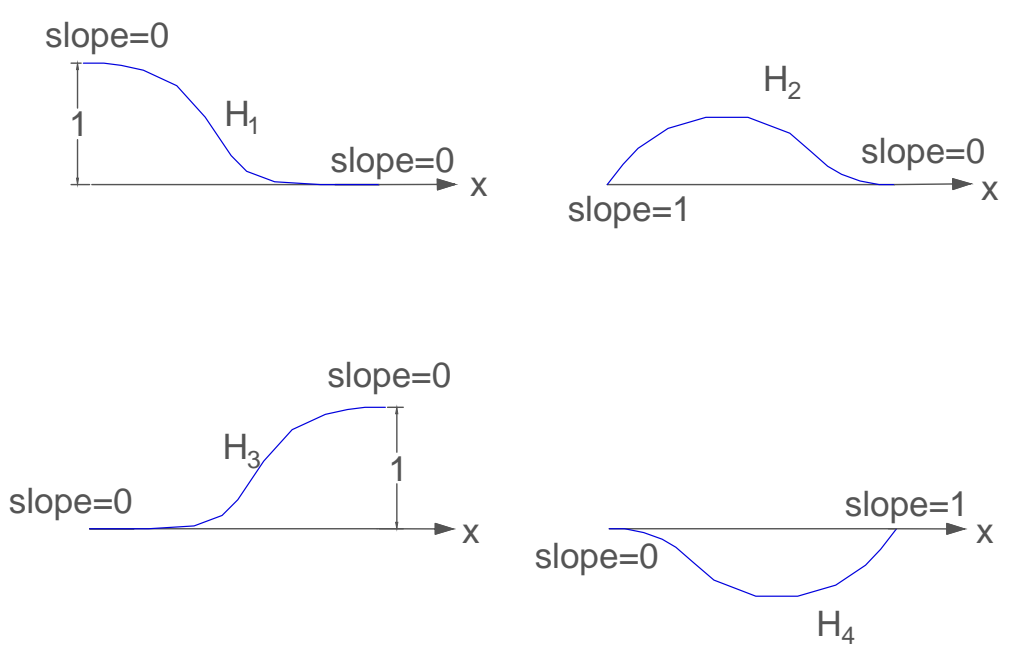

Figure 4.4: Hermitian shape functions

With

$$
\begin{aligned}
& H_{1}=1-3 \frac{x^{2}}{l^{2}}+2 \frac{x^{3}}{l^{3}} \\
& H_{2}=x-2 \frac{x^{2}}{l}+\frac{x^{3}}{l^{2}} \\
& H_{3}=3 \frac{x^{2}}{l^{2}}-2 \frac{x^{3}}{l^{3}} \\
& H_{4}=-\frac{x^{2}}{l}+\frac{x^{3}}{l^{2}}
\end{aligned}
$$

the deflections and virtual deflections are constructed using the above shape functions as 


$$
\begin{aligned}
y^{e}(x, t) & =H_{1} y_{1}+H_{2} y_{1}^{\prime}+H_{3} y_{2}+H_{4} y_{2}^{\prime} \\
\delta y^{e}(x) & =H_{1} \delta y_{1}+H_{2} \delta y_{1}^{\prime}+H_{3} \delta y_{2}+H_{4} \delta y_{2}^{\prime}
\end{aligned}
$$

Substituting Equations 4.3.4 into Equation 4.3.1 gives

$$
\sum_{i=1}^{4} \delta y_{i}\left\{\sum_{j=1}^{4}\left[\int_{0}^{l^{e}} E I H_{i}^{\prime \prime} H_{j}^{\prime \prime} d x\right] y_{j}\right\}
$$

The above equation is valid for any virtual deflection $\delta y$ hence we have,

$$
\sum_{j=1}^{4}\left[\int_{0}^{l^{e}} E I H_{i}^{\prime \prime} H_{j}^{\prime \prime} d x\right] y_{j}
$$

Now, the bending stiffness matrix contribution may be written as

$$
\boldsymbol{K}^{e}=\int_{0}^{l^{e}} E I H_{i}^{\prime \prime} H_{j}^{\prime \prime} d x
$$

and upon simplification, we have

$$
\boldsymbol{K}^{e}=\frac{E I}{l^{3}}\left[\begin{array}{cccc}
12 & 6 l & -12 & 6 l \\
6 l & 4 l^{2} & -6 l & 2 l^{2} \\
-12 & -6 l & 12 & -6 l \\
6 l & 2 l^{2} & -6 l & 4 l^{2}
\end{array}\right]
$$

For the centrifugal force term, we have

$$
\int_{0}^{l} \delta y \rho A v^{2} y^{\prime \prime} d x=0
$$

Using the same shape functions in Equation 4.3.3, and substituting Equation 4.3.4 into Equation 4.3.9, we have,

$$
\sum_{i=1}^{4} \delta y_{i}\left\{\sum_{j=1}^{4}\left[\int_{0}^{l^{e}} \rho A v^{2} H_{i} H_{j}^{\prime \prime} d x\right] y_{j}\right\}
$$

Equation 4.3 .10 is valid for any virtual deflection $\delta y$, hence the centrifugal contribution may be written as

$$
\boldsymbol{D}^{e}=\int_{0}^{l^{e}} \rho A v^{2} H_{i} H_{j}^{\prime \prime} d x
$$


Upon simplification, we have

$$
\boldsymbol{D}^{e}=\frac{\rho A v^{2}}{30 l}\left[\begin{array}{cccc}
-36 & -3 l & 36 & -3 l \\
-33 l & -4 l^{2} & 3 l & l^{2} \\
36 & 3 l & -36 & 3 l \\
-3 l & l^{2} & 33 l & -4 l^{2}
\end{array}\right]
$$

The Coriolis force term results in

$$
\int_{0}^{l} \delta y 2 \rho A v \frac{\partial^{2} y}{\partial x \partial t} d x=0
$$

In a similar manner, using the same shape functions and substituting Equation 4.3.4 into Equation 4.3.13, we obtain the Coriolis contribution as

$$
\boldsymbol{C}^{e}=\int_{0}^{l^{e}} 2 \rho A v H_{i} H_{j}^{\prime} d x
$$

Upon simplification, we have

$$
\boldsymbol{C}^{e}=\frac{\rho A v}{30}\left[\begin{array}{cccc}
-30 & -6 l & -30 & 6 l \\
6 l & 0 & -6 l & l^{2} \\
30 & 6 l & 30 & -6 l \\
-6 l & -l^{2} & 6 l & 0
\end{array}\right]
$$

Finally, we have the inertia term which is

$$
\int_{0}^{l} \delta y(m+\rho A) \frac{\partial^{2} y}{\partial t^{2}} d x=0
$$

From Equations 4.3.3, 4.3.4, 4.3.16, and simplifying as above, we have that

$$
\boldsymbol{M}^{e}=\int_{0}^{l^{e}}(m+\rho A) H_{i} H_{j} d x
$$

which results in

$$
\boldsymbol{M}^{e}=\frac{(m+\rho A) l}{420}\left[\begin{array}{cccc}
156 & 22 l & 54 & -13 l \\
22 l & 4 l^{2} & 13 l & -3 l^{2} \\
54 & 13 l & 156 & -22 l \\
-13 l & -3 l^{2} & -22 l & 4 l^{2}
\end{array}\right]
$$


Hence, from Equation 4.2.10 we have

$$
\sum_{e=1}^{n e l}\left(\boldsymbol{K}^{e} y^{e}+\boldsymbol{D}^{e} y^{e}+\boldsymbol{C}^{e} \dot{y}^{e}+\boldsymbol{M}^{e} \ddot{y}^{e}\right)=0
$$

where $\boldsymbol{K}^{e}, \boldsymbol{D}^{e}, \boldsymbol{C}^{e}, \boldsymbol{M}^{e}$, are matrices representing the bending stiffness, centrifugal, Coriolis, and inertia terms respectively.

\subsection{Time stepping scheme}

Various solution methods for time dependent problems which can be explicit or implicit in nature are available in literature, including but not limited to the central difference, Adams, Runge-Kutta, and Newmark family of algorithms. Here the generalised mid-point rule for the discretisation of time is used, where we solve for the deflection $y_{n+1}$ at time $t_{n+1}$ using deflection, velocity and acceleration at time $t_{n+\gamma}$, with $\frac{1}{2} \leq \gamma \leq 1$.

The choice of the parameter $\gamma$ affects the accuracy as well as the amount of numerical damping of the solution. With $\gamma=0$ we have the forward Euler method which is fully explicit. Using $\gamma=1$ results in a first order accurate solution with strong numerical damping known as the backward Euler method. The trapezoidal(midpoint) rule which is second order accurate with no numerical damping is obtained when we choose $\gamma=\frac{1}{2}$.

We compute the deflection, velocity and acceleration at time $t_{n+\gamma}$ with

$$
\begin{aligned}
& y_{n+\gamma}=\gamma y_{n+1}+(1-\gamma) y_{n} \\
& \dot{y}_{n+\gamma}=\gamma \dot{y}_{n+1}+(1-\gamma) \dot{y}_{n}=\frac{y_{n+1}-y_{n}}{\Delta t} \\
& \ddot{y}_{n+\gamma}=\frac{\dot{y}_{n+1}-\dot{y}_{n}}{\Delta t}
\end{aligned}
$$

rearranging Equation 4.4 .1 for $\ddot{y}_{n+\gamma}$ we have

$$
\begin{aligned}
& \dot{y}_{n+1}=\frac{y_{n+1}-y_{n}}{\gamma \Delta t}-\frac{1-\gamma}{\gamma} \dot{y}_{n} \\
& \ddot{y}_{n+\gamma}=\frac{y_{n+1}-y_{n}}{\gamma \Delta t^{2}}-\frac{1}{\gamma \Delta t} \dot{y}_{n}
\end{aligned}
$$

With Equations 4.4.1, 4.4.2, 4.3.2 and the equation of motion (Equation 4.3.19, 
we obtain the element force vector $\boldsymbol{f}^{e}$

$$
\boldsymbol{f}^{e}=\left(\boldsymbol{K}^{e}+\boldsymbol{D}^{e}\right) \boldsymbol{y}_{n+\gamma}^{e}+\boldsymbol{C}^{e} \dot{\boldsymbol{y}}_{n+\gamma}^{e}+\boldsymbol{M}^{e} \ddot{\boldsymbol{y}}_{n+\gamma}^{e}
$$

Rearranging Equation 4.4.3, we have

$$
\begin{aligned}
\boldsymbol{f}^{e}= & \left(\left(\boldsymbol{K}^{e}+\boldsymbol{D}^{e}\right) \gamma+\boldsymbol{C}^{e} \frac{1}{\Delta t}+\boldsymbol{M}^{e} \frac{1}{\gamma \Delta t^{2}}\right) \boldsymbol{y}_{n+1}^{e} \\
& +\left(\left(\boldsymbol{K}^{e}+\boldsymbol{D}^{e}\right)(1-\gamma)-\boldsymbol{C}^{e} \frac{1}{\Delta t}-\boldsymbol{M}^{e} \frac{1}{\gamma \Delta t^{2}}\right) \boldsymbol{y}_{n}^{e} \\
& -\boldsymbol{M}^{e} \frac{1}{\gamma \Delta t} \dot{\boldsymbol{y}}_{n}^{e}
\end{aligned}
$$

The element tangent operator follows directly from Equation 4.4 .4 as

$$
\left(\boldsymbol{K}^{e}+\boldsymbol{D}^{e}\right) \gamma+\boldsymbol{C}^{e} \frac{1}{\Delta t}+\boldsymbol{M}^{e} \frac{1}{\gamma \Delta t^{2}}
$$

In the usual manner, the Global system $\boldsymbol{A} \boldsymbol{y}_{n+1}=\boldsymbol{b}$ is assembled from the elements and solved for the required degrees of freedom (deflection and rotation) at the nodes. Although not required, the Newton-Raphson method presented in previous Chapters was used to solve the global system, convergence was achieved in only 1 iteration.

\subsection{Numerical examples}

To study the dynamic behaviour of a linear system, first we consider the eigenfrequencies of two main classes of problems, pipes with supported ends, and cantilevered pipes. The critical velocity for onset of instability is determined by varying the flow velocity, and is compared against theoretical results available in literature for both cases. For these examples, the circular pipe with inner and outer diameters $d_{i}=32 \mathrm{~mm}$ and $d_{o}=70 \mathrm{~mm}$ respectively, is of length $L=2 \mathrm{~m}$, Young's modulus $E=2.5 \times 10^{7} \mathrm{Nm}^{-2}$, and pipe density $\rho_{p}=1167 \mathrm{kgm}^{-3}$. Solutions and results are available in e.g. [10, 16, 48] among others.

\subsubsection{Natural frequency of vibration}

The first example, shown in Figure 4.6 is a simply supported pipe displaced initially in the middle and allowed to undergo free vibration in the absence of fluid flow. 


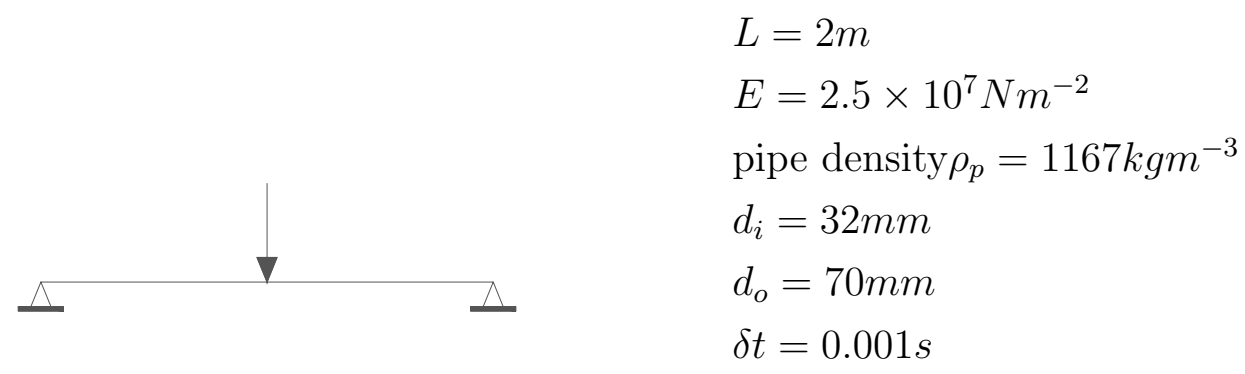

Figure 4.5: Geometry

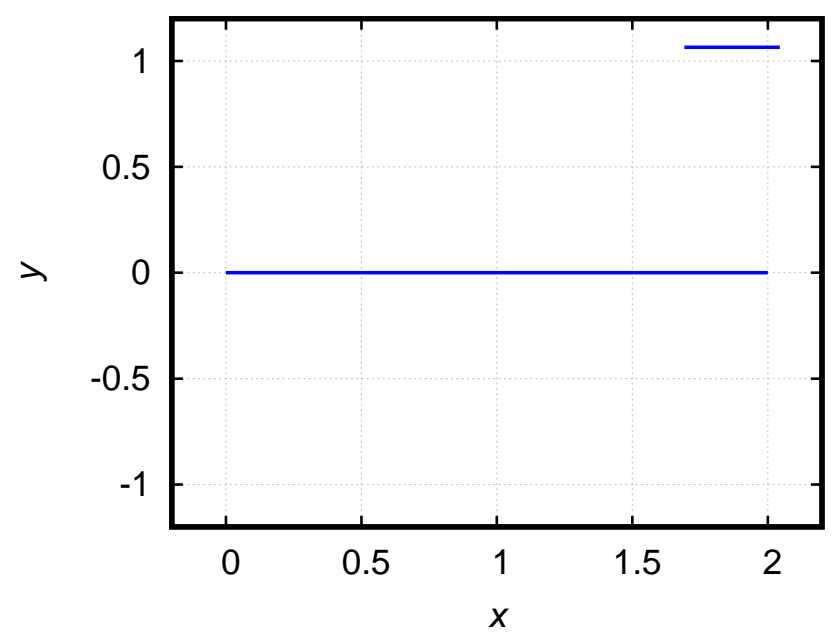

Figure 4.6: Undeformed configuration of the pipe

With $m=\rho_{p} A_{p}$, we recover the fundamental natural frequency of vibration to be $\omega_{n}=6.93$ which matches the analytical value of

$$
\omega_{n}=\frac{\pi^{2}}{L^{2}}\left(\frac{E I}{m}\right)^{\frac{1}{2}}=6.95
$$

The deformed pipe at various time steps is shown in Figure 4.7, and Figure4.8 shows the displacement history of the mid-span of the pipe which has been obtained with 20 finite elements.

For a cantilevered pipe on the other hand, the fundamental natural frequency is given as

$$
\omega_{n}=\frac{1.8751^{2}}{L^{2}}\left(\frac{E I}{m}\right)^{\frac{1}{2}}=2.476
$$

which matches $\omega_{n}=2.474$ recovered from the simulation. 


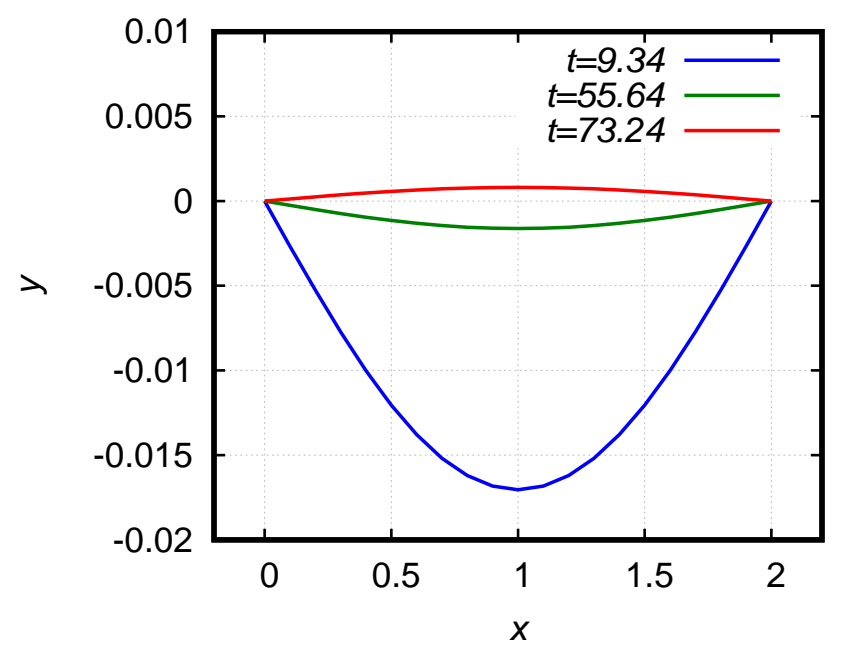

Figure 4.7: Deformed configuration of the pipe

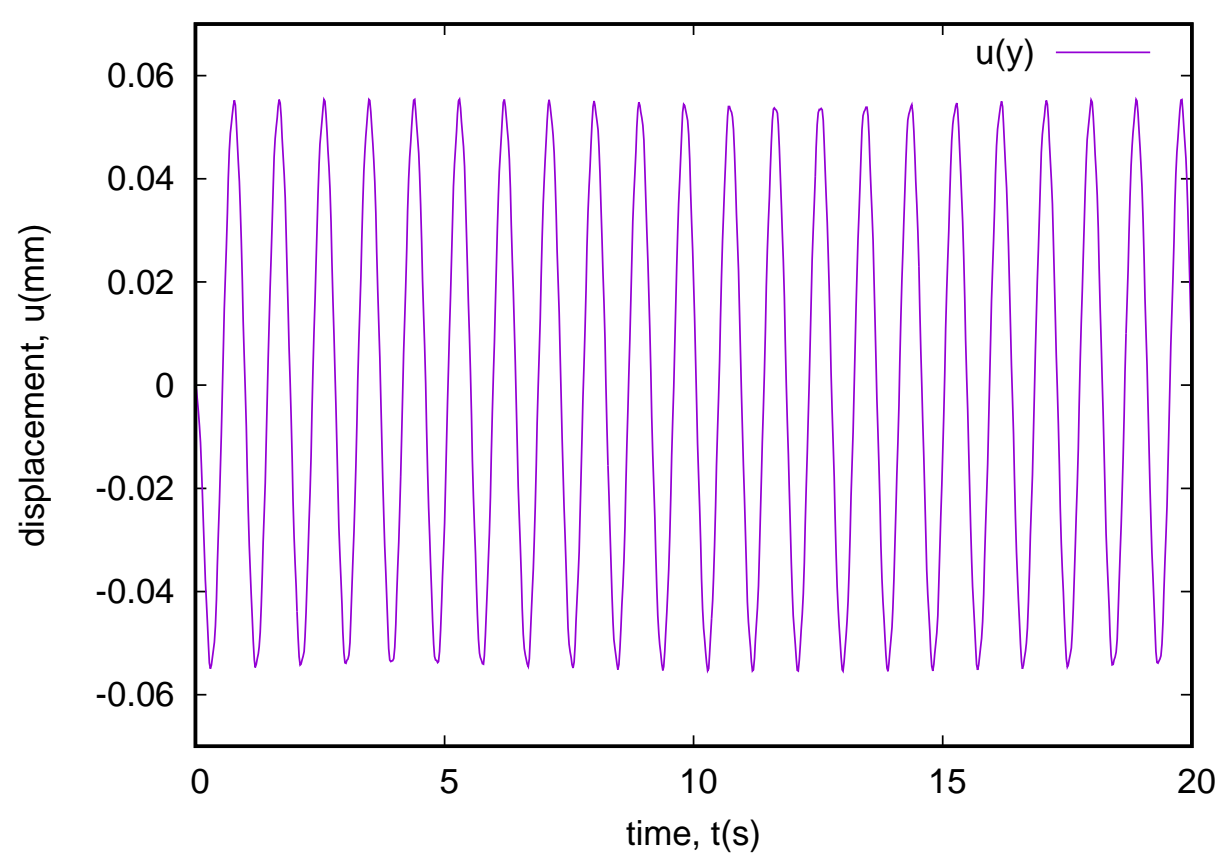

Figure 4.8: Displacement history of the mid-span of a simply supported pipe undergoing free vibration

$$
\begin{aligned}
& L=2 \mathrm{~m} \\
& E=2.5 \times 10^{7} \mathrm{Nm}^{-2} \\
& \text { pipe density } \rho_{p}=1167 \mathrm{kgm}^{-3} \\
& d_{i}=32 \mathrm{~mm} \\
& d_{o}=70 \mathrm{~mm} \\
& \delta t=0.001 \mathrm{~s}
\end{aligned}
$$




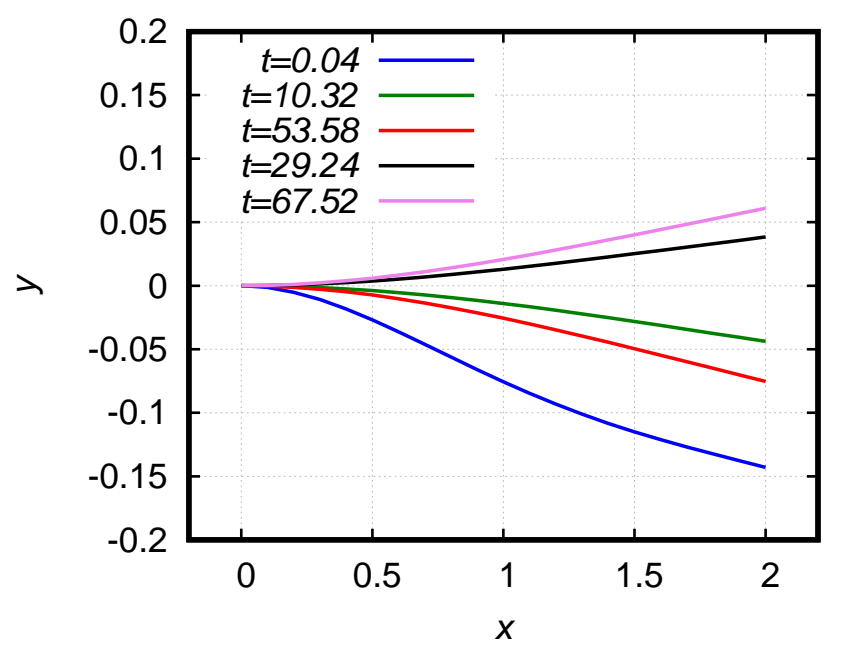

Figure 4.10: Deformed configuration of the pipe

The deformed pipe at various time instances is shown in Figure 4.10, and The free-end displacement history is shown in Figure 4.11 below, also obtained with 20 finite elements.

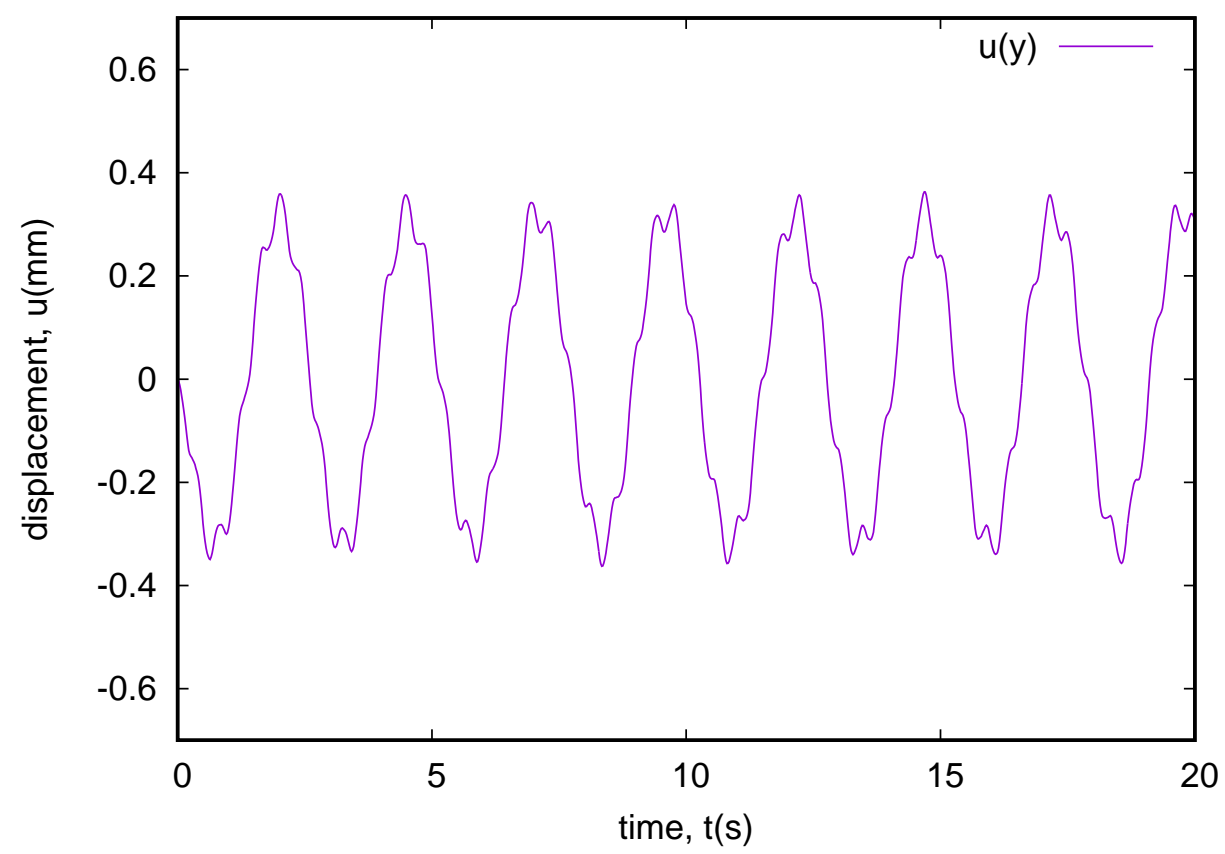

Figure 4.11: Displacement history of the free end of a cantilever pipe undergoing free vibration 


\subsubsection{Stability of pipes with simply supported ends}

For pipes with simply supported ends, as velocity $v$ of fluid flow with density $\rho_{f}=999 \mathrm{kgm}^{-3}$ through the pipe increases, the natural frequencies $\omega$ decrease, and becomes zero when the flow velocity equals the critical velocity $v_{c}$. At this point, the pipe buckles, and this instability is due to the centrifugal force term.

The expected response of the system is observed, as Figure4.13 shows good agreement with the theoretical reduction of fundamental frequency with increasing flow velocity given by the relationship

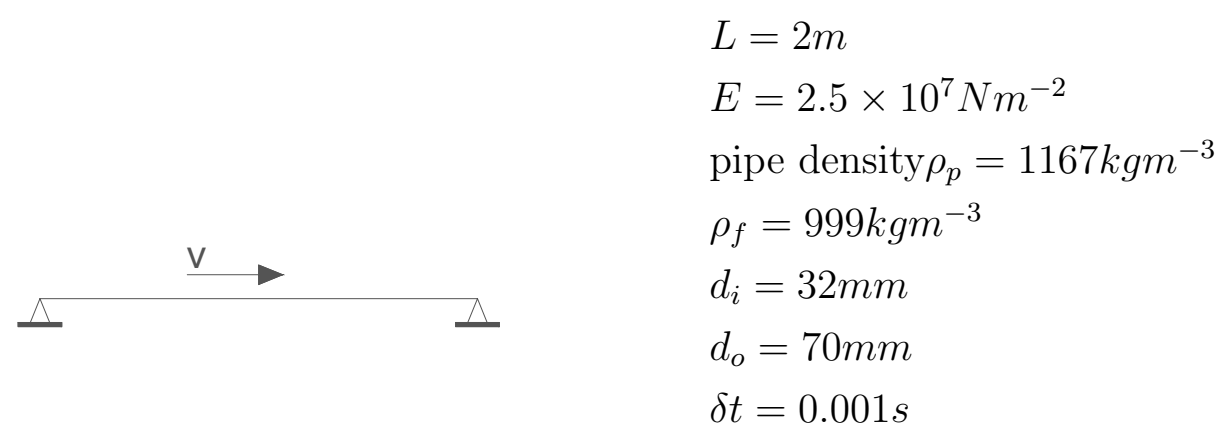

Figure 4.12: Geometry

$$
\frac{\omega}{\omega_{n}}=\left(1-\left[\frac{v}{v_{c}}\right]^{2}\right)^{\frac{1}{2}}
$$

with the critical velocity

$$
v_{c}=\frac{\pi}{L}\left(\frac{E I}{\rho A}\right)^{\frac{1}{2}}=9.3022, \quad \rho A=\rho_{f} A_{f}
$$

The value of $v_{c}=9.3$ was obtained for the critical velocity with 20 finite elements, Figure 4.14 and Figure 4.15 show deformed pipe, and the mid-span displacement histories of the pipe for different flow velocities $v_{i}<v_{c}$ respectively.

\subsubsection{Stability of cantilever pipes}

For cantilever pipes on the other hand, instability is associated with the coriolis force term, and the pipe does not buckle but flutters, flailing about with finite frequency. The critical flow velocity at onset of instability as a function of mass ratio is given 


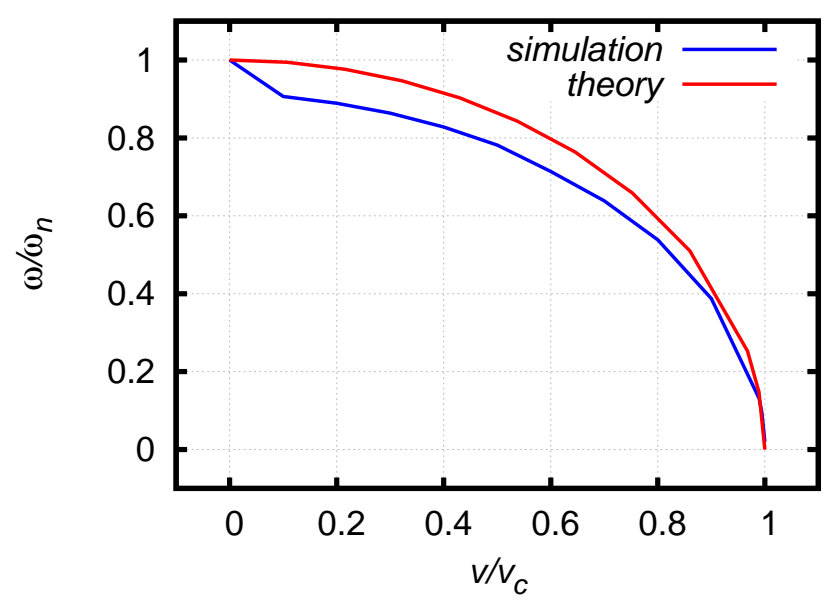

Figure 4.13: Frequency reduction with increasing flow velocity

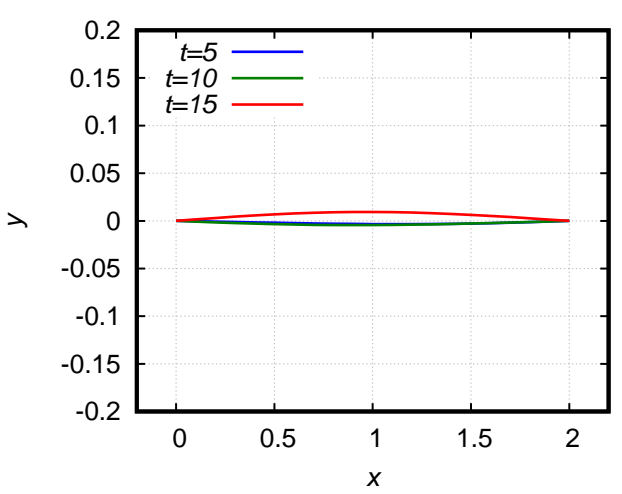

(a) $v=3.7208$

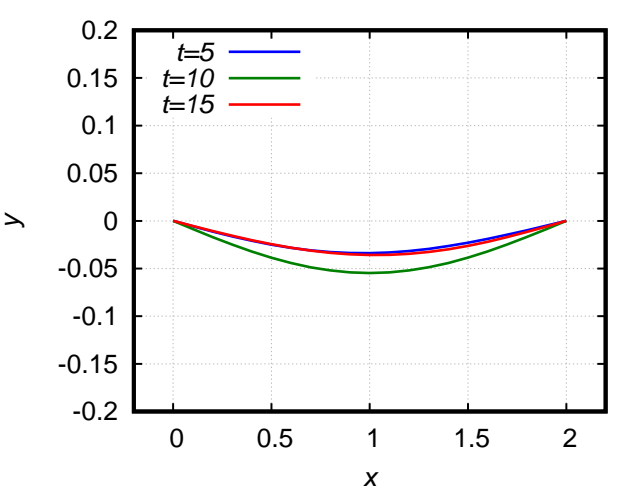

(b) $v=8.3718$

Figure 4.14: Displacement history of the mid-span of a simply supported pipe for various flow velocities

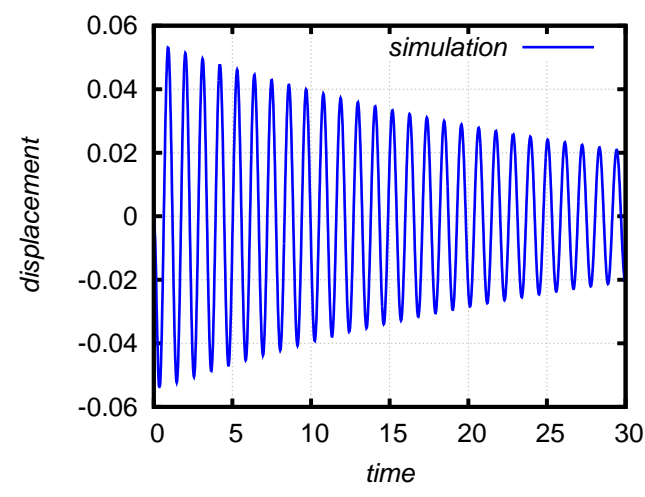

(a) $v=3.7208$

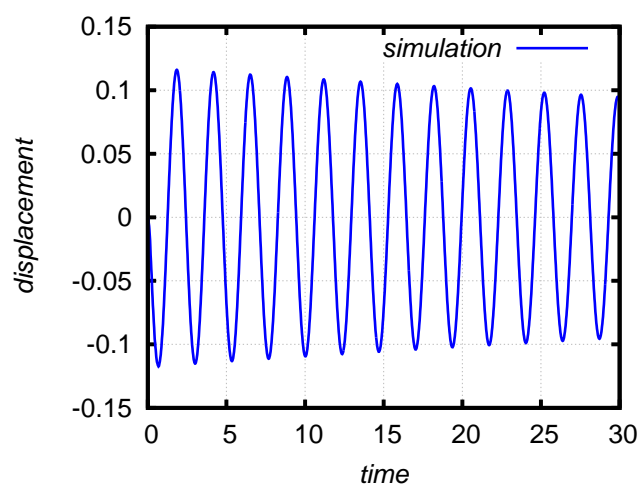

(b) $v=8.3718$

Figure 4.15: Displacement history of the mid-span of a simply supported pipe for various flow velocities

as

$$
M=\rho A+m, \quad \frac{\rho A}{M}=0.184
$$




$$
v_{c} L\left(\frac{\rho A}{E I}\right)^{\frac{1}{2}} \approx 5.5 \quad v_{c} \approx 16.285
$$

With 20 finite elements, we obtain a critical velocity of approximately 16.23 and Figure 4.17 shows the free-end displacement histories for different flow velocities.

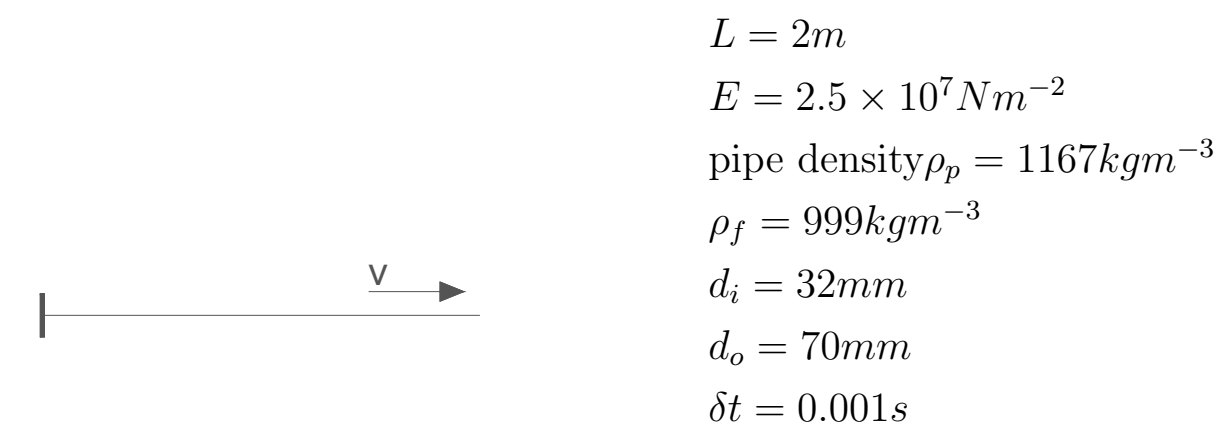

Figure 4.16: Geometry

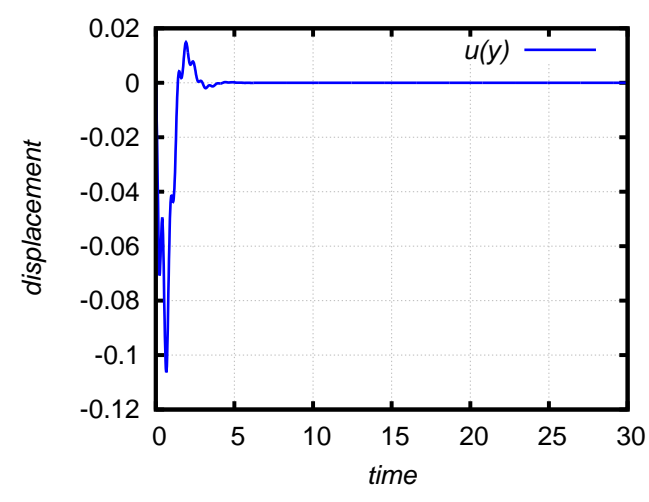

(a) $v=7.5$

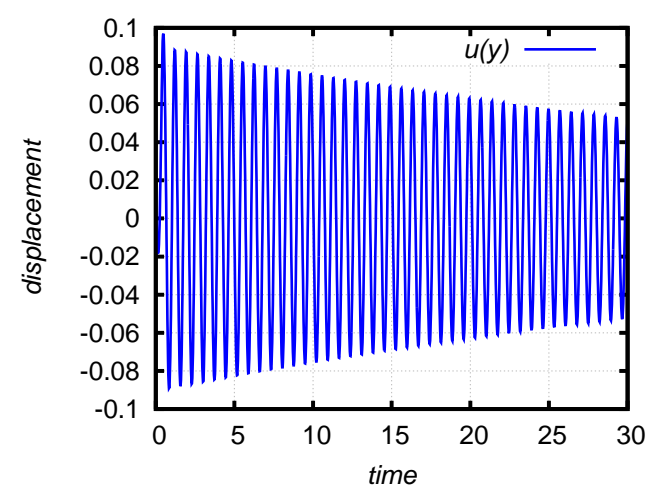

(c) $v=16.2$

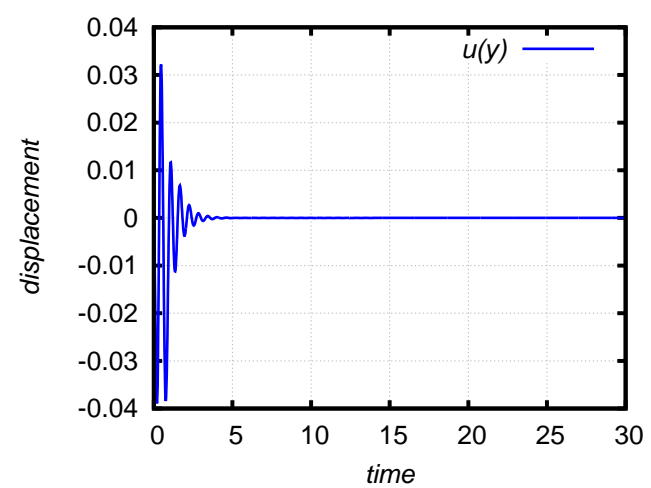

(b) $v=12$

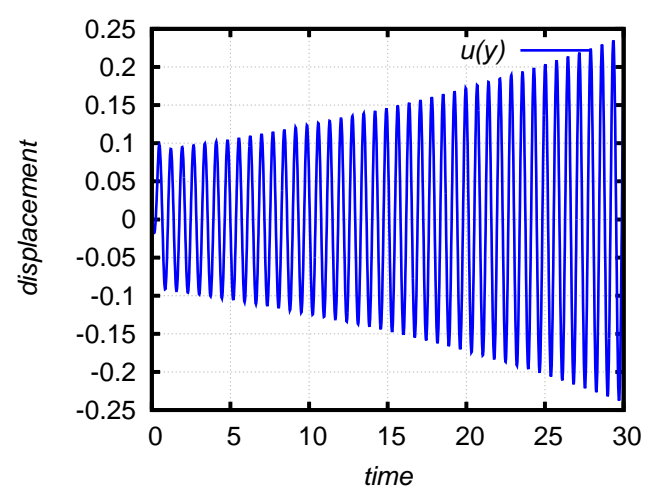

(d) $v=16.3$

Figure 4.17: Displacement history of the free end of a cantilever pipe for various flow velocities

The deformed pipe at various time steps for flow velocities around the critical velocity $v c \approx 16.23$ is presented in Figure 4.18 . 


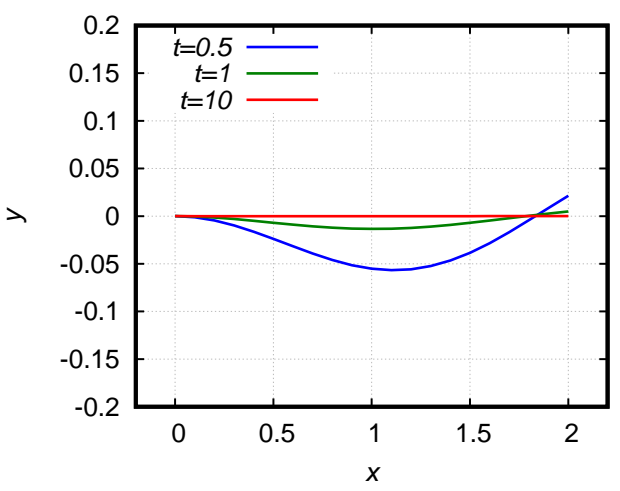

(a) $v=12$

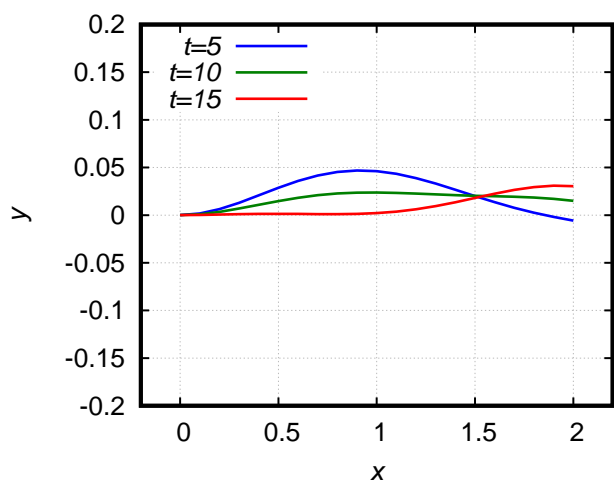

(b) $v=16.2$

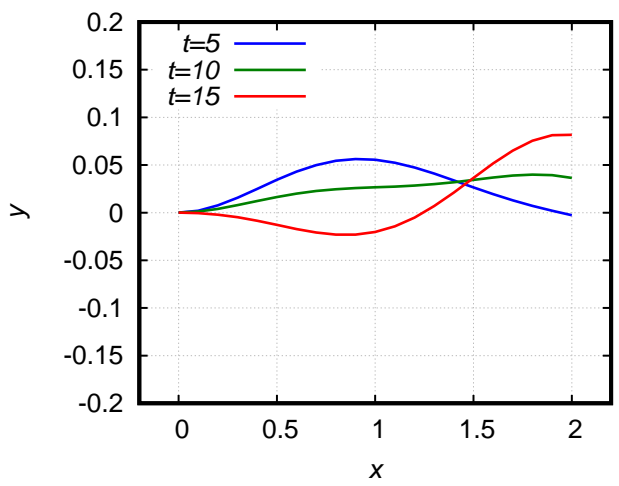

(c) $v=16.3$

Figure 4.18: Deformed cantilever pipes conveying fluid at velocities around the critical velocity

\subsection{Conclusion}

The 2D linear case is well understood, numerical and analytical solutions are readily available, but this is by no means a trivial exercise. This serves as a starting point for validation of results as we have excellent agreement with existing examples. Simulations with the nonlinear code, for small deflection cases will be compared against those in this section, before moving on to systems from which we expect more interesting responses from the next chapter of this work.

The results obtained from simulations for the fundamental frequencies of vibration for both the cantilever and simply supported pipe are in excellent agreement with the analytically computed results. The next examples go on to simulate flow through the pipe, increasing the velocity of flow, till we reach the respective critical velocities, at which point, a nonlinear element is required to simulate flows beyond the critical velocity. The obtained critical velocities matched those available in literature, and the expected reduction of fundamental frequency in the simply supported case, is observed.

These results show the accuracy of the element and will also serve as a point of 
comparison for small deformations of the nonlinear element presented in the next chapter of this work. 


\section{Chapter 5}

\section{Pipes Conveying fluid: 2D nonlinear solution}

\subsection{Introduction}

Building upon the linear element presented in the previous chapter, here we present the finite element solution of non-linear problems. We observe the expected behaviour of the pipe as seen in the examples presented, and the various components which make up the system of equations are presented. The examples are compared against analytical results, as well as results for the linear case where applicable, and we have excellent agreement.

\subsection{Variational formulation}

\subsubsection{Pipe stiffness term}

From Wriggers [61], for the stiffness term which is independent of flow, the geometrically exact formulation is chosen for its ability to handle large deflections and rotations. Here we present an initially straight beam with coinciding local and global axes. For an arbitrary orientation of the beam in space, an additional transformation is applied, see also Figure 5.1. Based on the kinematical assumption for the beam deformation

$$
\boldsymbol{\varphi}=\left\{\begin{array}{c}
X_{1}+u \\
v
\end{array}\right\}+X_{2}\left\{\begin{array}{c}
-\sin \theta \\
\cos \theta
\end{array}\right\}
$$

By using the principle of virtual work the strain-deflection relations were derived, leading to the strain measures for the axial and shear strains, and curvature 


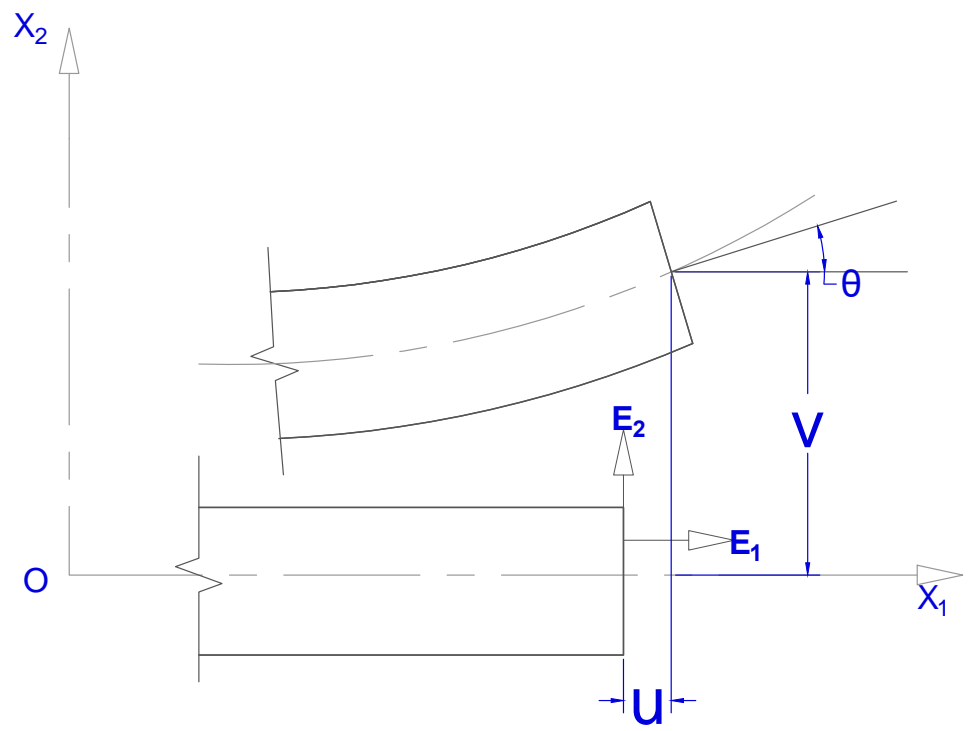

Figure 5.1: Beam Kinematics

$$
\begin{aligned}
\epsilon & =\left(1+u^{\prime}\right) \cos \theta+v^{\prime} \sin \theta-1 \\
\gamma & =v^{\prime} \cos \theta-\left(1+u^{\prime}\right) \sin \theta \\
\kappa & =\theta^{\prime}
\end{aligned}
$$

where $u$ is the displacement in the axial direction, $v$ is the deflection and $\theta$ is the rotation. $(\bullet)^{\prime}$ denotes the derivative with respect to the arc-length coordinate of the beam, $X_{1}$. The above nonlinear strain measures can be written in matrix notation

$$
\epsilon=\Lambda u^{\prime}-N
$$

where

$$
\boldsymbol{\epsilon}=\left\{\begin{array}{c}
\epsilon \\
\gamma \\
\kappa
\end{array}\right\}, \quad \boldsymbol{\Lambda}=\left[\begin{array}{ccc}
\cos \theta & \sin \theta & 0 \\
-\sin \theta & \cos \theta & 0 \\
0 & 0 & 1
\end{array}\right], \quad \boldsymbol{u}^{\prime}=\left\{\begin{array}{c}
1+u^{\prime} \\
v^{\prime} \\
\theta^{\prime}
\end{array}\right\}, \quad \boldsymbol{N}=\left\{\begin{array}{l}
1 \\
0 \\
0
\end{array}\right\}
$$

$\boldsymbol{\Lambda}$ here is the rotational tensor which serves the purpose of transforming the base 
vector $\left(E_{1}, E_{2}\right)$ in the reference configuration to to the base $\left(e_{1}, e_{2}\right)$ in the current configuration, and is the source of the nonlinearity.

From the principle of virtual work, we obtain the weak form of equilibrium which is stated as

$$
\int_{0}^{l}(N \delta \epsilon+Q \delta \gamma+M \delta \kappa) d x-W_{e x t}=0
$$

where $N, Q$, and $M$ are the stress resultants, and introducing the vector $\boldsymbol{S}=$ $\{N, Q, M\}^{T}$, we have Equation 5.2.4 in compact form as

$$
\int_{0}^{l} \delta \boldsymbol{\epsilon}^{T} \boldsymbol{S} d x-W_{e x t}=0
$$

The variation of the strains yields

$$
\delta \boldsymbol{\epsilon}=\boldsymbol{\Lambda} \boldsymbol{\eta}^{\prime}+\frac{\partial \boldsymbol{\Lambda}}{\partial \theta} \boldsymbol{u}^{\prime} \delta \theta
$$

where $\boldsymbol{\eta}=\{\delta u, \delta v, \delta \theta\}^{T}$, and inserting Equation 5.2.6 into the weak form (Equation 5.2.5) we have

$$
\int_{0}^{l}\left[\boldsymbol{\eta}^{T} \boldsymbol{\Lambda}^{T}+\delta \theta \boldsymbol{u}^{\prime T}\left(\frac{\partial \boldsymbol{\Lambda}}{\partial \theta}\right)^{T}\right] \boldsymbol{S} d x-W_{e x t}=0
$$

which is the stress divergence term.

While large deflections and rotations are expected, for most applications, which our problem falls under, the strains are small. Hence the constitution may be expressed by a linear elastic relation between the strains and the Piola-Kirchhoff stresses. In compact form, we have that

$$
\boldsymbol{S}=\boldsymbol{D} \boldsymbol{\epsilon} ; \quad \boldsymbol{D}=\left[\begin{array}{ccc}
E A & 0 & 0 \\
0 & \hat{\kappa} G A & 0 \\
0 & 0 & E I
\end{array}\right]
$$

where $E=$ Young's modulus, $A=$ cross-sectional area, $G=$ shear modulus, $I=$ moment of inertia, and here $\hat{\kappa}=$ shear correction factor.

\subsubsection{Centrifugal force term}

The second term is the centrifugal force contribution, and we have

$$
f_{c}=\rho A v^{2} \frac{\delta}{\delta s^{2}}\left\{\begin{array}{l}
x \\
y
\end{array}\right\}
$$


Applying the principle of virtual work and integrating by parts, we have for an element

$$
\begin{gathered}
\int_{e} \delta y \rho A v^{2} y^{\prime \prime} d s=\rho A v^{2} \int_{e} \delta y y^{\prime \prime} d s \\
=\rho A v^{2}\left(\left[\delta y^{\prime}\right]_{0}^{l}-\int_{e} \delta y^{\prime} y^{\prime} d s\right) \\
\frac{\delta x}{\delta s}=\frac{\Delta x}{l}, \quad \frac{\delta y}{\delta s}=\frac{\Delta y}{l}
\end{gathered}
$$

the contribution of the second term in the above equation is added to all elements of the pipe, and the first term is applied at the ends of the pipe, hence the first and last element.

\subsubsection{Inertia term}

For the inertia term, from Equation 4.3.17 we have

$$
\int_{0}^{l}(m+\rho A)(x) \ddot{y}(x, t) \delta y(x) d x
$$

\subsubsection{Coriolis force term}

For the Coriolis term, we have

$$
f_{r}=2 \rho A v \frac{\delta}{\delta s \delta t}\left\{\begin{array}{l}
x \\
y
\end{array}\right\}
$$

\subsection{Finite element formulation}

\subsubsection{Pipe stiffness term}

For a finite element approximation, the beam is divided into elements and shape functions are introduced and used to construct the element axial displacement, deflection, and rotation. This is given by

$$
\left\{\begin{array}{l}
u^{e} \\
v^{e} \\
\theta^{e}
\end{array}\right\}=\sum_{a=1}^{n}\left[\begin{array}{ccc}
N_{a} & 0 & 0 \\
0 & N_{a} & 0 \\
0 & 0 & N_{a}
\end{array}\right]\left\{\begin{array}{l}
u_{a} \\
v_{a} \\
\theta_{a}
\end{array}\right\}
$$

where $n$ is the number of element nodes. For a 2-noded element, we have that 

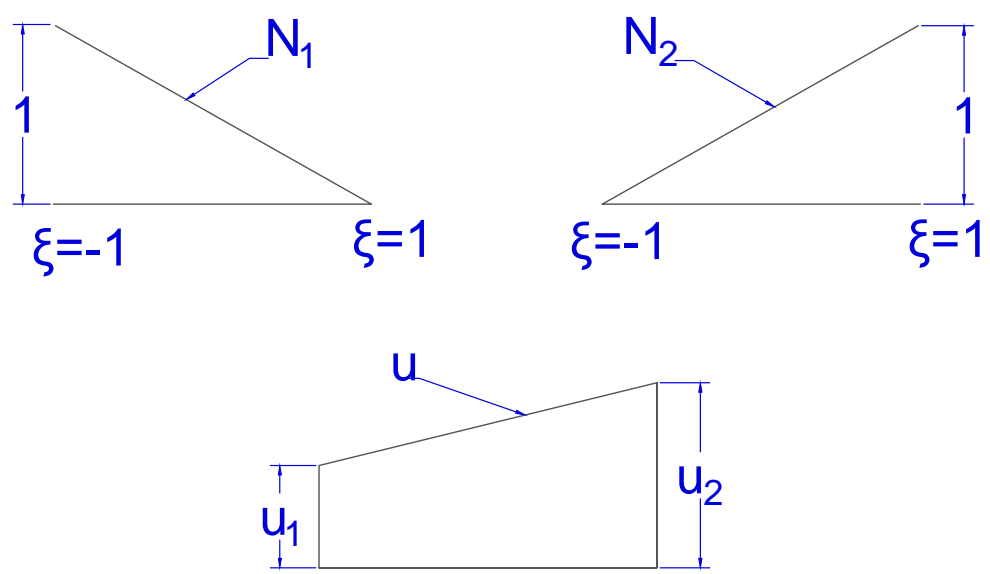

Figure 5.2: 2 node linear shape functions and interpolation

$$
\begin{aligned}
N_{1} & =\frac{1-\xi}{2} \\
N_{2} & =\frac{1+\xi}{2} \\
\text { and } \quad d x & =\frac{l_{e}}{2} d \xi
\end{aligned}
$$

Using the above approximation, the variation of the strains are expressed as

$$
\delta \epsilon^{e}=\sum_{a=1}^{n} B_{a} \eta_{a} \quad \text { where } B_{a}=\left[\begin{array}{ccc}
N_{a}^{\prime} \cos \theta^{e} & N_{a}^{\prime} \sin \theta^{e} & \alpha_{1} N_{a} \\
-N_{a}^{\prime} \sin \theta^{e} & N_{a}^{\prime} \cos \theta^{e} & \alpha 2 N_{a} \\
0 & 0 & N_{a}^{\prime}
\end{array}\right]
$$

and

$$
\begin{aligned}
& \alpha_{1}=-\left(1+u_{e}^{\prime}\right) \sin \theta^{e}+v_{e}^{\prime} \cos \theta^{e} \\
& \alpha_{2}=-\left(1+u_{e}^{\prime}\right) \cos \theta^{e}-v_{e}^{\prime} \sin \theta^{e}
\end{aligned}
$$


The first term in Equation 5.2 .5 gives rise to the residual as

$$
R_{a}=\int_{0}^{l_{e}} B_{a}^{T} S^{e} d x=\int_{-1}^{+1} B_{a}^{T}(\xi) S^{e}(\xi) \frac{l^{e}}{2} d \xi
$$

For a 2-node element, one quadrature point is sufficient for the integration as the shear term is underintegrated and is advantageous as it prevents 'shear locking'.

To achieve quadratic convergence of the Newton method, we require a linearisation of the weak form (Equation 5.2.4). Application of the directional derivative in a standard manner yields

$$
\int_{0}^{l}(\delta \epsilon E A \Delta \epsilon+\delta \gamma \hat{\kappa} G A \Delta \gamma+\delta \kappa E I \Delta \kappa) d x+\int_{0}^{l}(\Delta \delta \epsilon N+\Delta \delta \gamma Q+\Delta \delta \kappa M) d x
$$

By introducing the above finite element interpolations, the explicit form for the tangent stiffness matrix is given by

$$
\begin{aligned}
k_{a b} & =\int_{0}^{l}\left(B_{a}^{T} D B_{b}+N G_{a b}^{N}+Q G_{a b}^{Q}\right) d x \\
\boldsymbol{K}^{\boldsymbol{e}} & =\int_{-1}^{+1}\left(B_{a}^{T} D B_{b}+N G_{a b}^{N}+Q G_{a b}^{Q}\right) \frac{l^{e}}{2} d \xi
\end{aligned}
$$

The matrices $G_{a b}^{N}$ and $G_{a b}^{Q}$ are given by

$$
\begin{aligned}
& G_{a b}^{N}=\left[\begin{array}{ccc}
0 & 0 & -N_{a}^{\prime} N_{b} \sin \theta^{e} \\
0 & 0 & N_{a}^{\prime} N_{b} \cos \theta^{e} \\
-N_{a} N_{b}^{\prime} \sin \theta^{e} & N_{a} N_{b}^{\prime} \cos \theta^{e} & \alpha_{3} N_{a} N_{b}
\end{array}\right] \\
& G_{a b}^{Q}=\left[\begin{array}{ccc}
0 & 0 & -N_{a}^{\prime} N_{b} \cos \theta^{e} \\
0 & 0 & -N_{a}^{\prime} N_{b} \sin \theta^{e} \\
-N_{a} N_{b}^{\prime} \cos \theta^{e} & -N_{a} N_{b}^{\prime} \sin \theta^{e} & \alpha_{4} N_{a} N_{b}
\end{array}\right]
\end{aligned}
$$

with

$$
\begin{aligned}
& \alpha_{3}=-\left(1+u_{e}^{\prime}\right) \cos \theta^{e}-v_{e}^{\prime} \sin \theta^{e} \\
& \alpha_{4}=\left(1+u_{e}^{\prime}\right) \sin \theta^{e}-v_{e}^{\prime} \cos \theta^{e}
\end{aligned}
$$




\subsubsection{Centrifugal force term}

Using a 2-node linear finite element we have the shape functions

$$
\begin{aligned}
& N_{1}=1-x / l \\
& N_{2}=x / l
\end{aligned}
$$

for the second term

$$
[-\Delta x,-\Delta y, 0, \Delta x, \Delta y, 0]^{T} \cdot \frac{\rho A v^{2}}{l}
$$

which is premultiplied by -1 and added to all elements of the pipe, following from equation 1.

Similarly, for the first term, we have

$$
[-\Delta x,-\Delta y, 0,0,0,0]^{T} \cdot \frac{\rho A v^{2}}{l}
$$

to the first element, and

$$
[0,0,0, \Delta x, \Delta y, 0]^{T} \cdot \frac{\rho A v^{2}}{l}
$$

to the last element

The element tangent matrix is obtained by employing the directional derivative

\subsubsection{Inertia term}

Introducing the same interpolation functions as with the centrifugal term above (Figure 5.2) and simplifying, we obtain the consistent mass matrix as

$$
\begin{gathered}
\boldsymbol{M}^{e}=\int_{0}^{l^{e}}(m+\rho A) N_{a} N_{b} d x \\
\boldsymbol{M}^{e}=\frac{(m+\rho A) l}{6}\left[\begin{array}{cccccc}
2 & 0 & 0 & 1 & 0 & 0 \\
0 & 2 & 0 & 0 & 1 & 0 \\
0 & 0 & 0 & 0 & 0 & 0 \\
1 & 0 & 0 & 2 & 0 & 0 \\
0 & 1 & 0 & 0 & 2 & 0 \\
0 & 0 & 0 & 0 & 0 & 0
\end{array}\right]
\end{gathered}
$$




\subsubsection{Coriolis force term}

Introducing the finite element approximation and using 2-noded linear elements, we have

$$
\int_{e} 2 \rho A v \frac{\delta}{\delta t}\left\{\begin{array}{l}
\frac{\delta x}{\delta s} \\
\frac{\delta y}{\delta s}
\end{array}\right\} \cdot\left\{\begin{array}{l}
\delta x \\
\delta y
\end{array}\right\}
$$

Simplifying the above expression results in

$$
F_{r}^{e}=\rho A v l \cdot \frac{\delta}{\delta t}\left[\frac{\Delta x}{l}, \frac{\Delta y}{l}, 0, \frac{\Delta x}{l}, \frac{\Delta y}{l}, 0\right]^{T}
$$

The Newton-Raphson solution procedure is an iterative process which uses a solution estimate $\boldsymbol{x}_{k}$ at iteration $k$ to obtain a new value $\boldsymbol{x}_{k+1}=\boldsymbol{x}_{k}+\boldsymbol{u}$ in terms of an increment $\boldsymbol{u}$ by establishing the linear approximation

$$
\boldsymbol{R}\left(\boldsymbol{x}_{k+1}\right) \approx \boldsymbol{R}\left(\boldsymbol{x}_{k}\right)+D \boldsymbol{R}\left(\boldsymbol{x}_{k}\right)[\boldsymbol{u}]=\mathbf{0}
$$

employing the directional derivative and obtaining the tangent matrix $\boldsymbol{K}$, a linear set of equations is solved at each Newton-Raphson iteration as

$$
\boldsymbol{K}\left(\boldsymbol{x}_{k}\right) \boldsymbol{u}=-\boldsymbol{R}\left(\boldsymbol{x}_{k}\right) ; \quad \boldsymbol{x}_{k+1}=\boldsymbol{x}_{k}+\boldsymbol{u}
$$

The algorithm is was presented in Chapter 2, and the time stepping scheme used is the same as that used in the previous Chapter fo the finite element solution of the linear problem. To show quadratic convergence, we present tables of residuals for some examples shown below.

\subsection{Numerical examples}

The validation of the accuracy of results for the nonlinear response of the system begins with comparisons to the linear solution presented in the previous section. It is expected that under certain conditions, here at low flow velocities and for small deformation, the problem becomes 'linear'. First we present the eigenfrequencies of the pipes, then consider velocities $v<v_{c}$. Problems with finite deformation and velocity $v>v_{c}$ are then presented and compared against results available in literature. For these problems, the circular pipe with inner and outer diameters $d_{i}=32 \mathrm{~mm}$ and $d_{o}=70 \mathrm{~mm}$ respectively, is of length $L=2 \mathrm{~m}$, Young's modulus 
$E=2.5 \times 10^{7} \mathrm{Nm}^{-2}$, and pipe density $\rho_{p}=1167 \mathrm{kgm}^{-3}$.

\subsubsection{Natural frequency of vibration}

For a simply supported pipe displaced initially and allowed to undergo free vibration in the absence of fluid flow, the nonlinear response for infinitesimal deformation shows good agreement with the linear solution. We recover the fundamental natural frequency of vibration to be $\omega_{n}=2.1969$ for which the analytical value is

$$
\omega_{n}=\frac{\pi^{2}}{L^{2}}\left(\frac{E I}{m}\right)^{\frac{1}{2}}=2.1974
$$

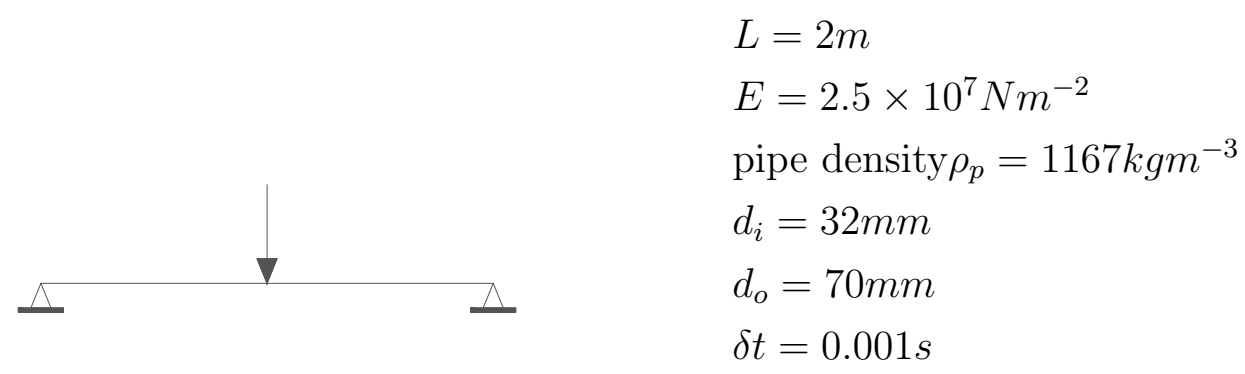

Figure 5.3: Geometry

Figure5.4 shows the displacement history of the mid-span of the pipe.

For a cantilevered pipe on the other hand, we have for the frequency

$$
\omega_{n}=\frac{1.8751^{2}}{L^{2}}\left(\frac{E I}{m}\right)^{\frac{1}{2}}=0.783
$$

$$
\begin{aligned}
& L=2 \mathrm{~m} \\
& E=2.5 \times 10^{7} \mathrm{Nm}^{-2} \\
& \text { pipe density } \rho_{p}=1167 \mathrm{kgm}^{-3} \\
& d_{i}=32 \mathrm{~mm} \\
& d_{o}=70 \mathrm{~mm} \\
& \delta t=0.001 \mathrm{~s}
\end{aligned}
$$

Figure 5.5: Geometry 


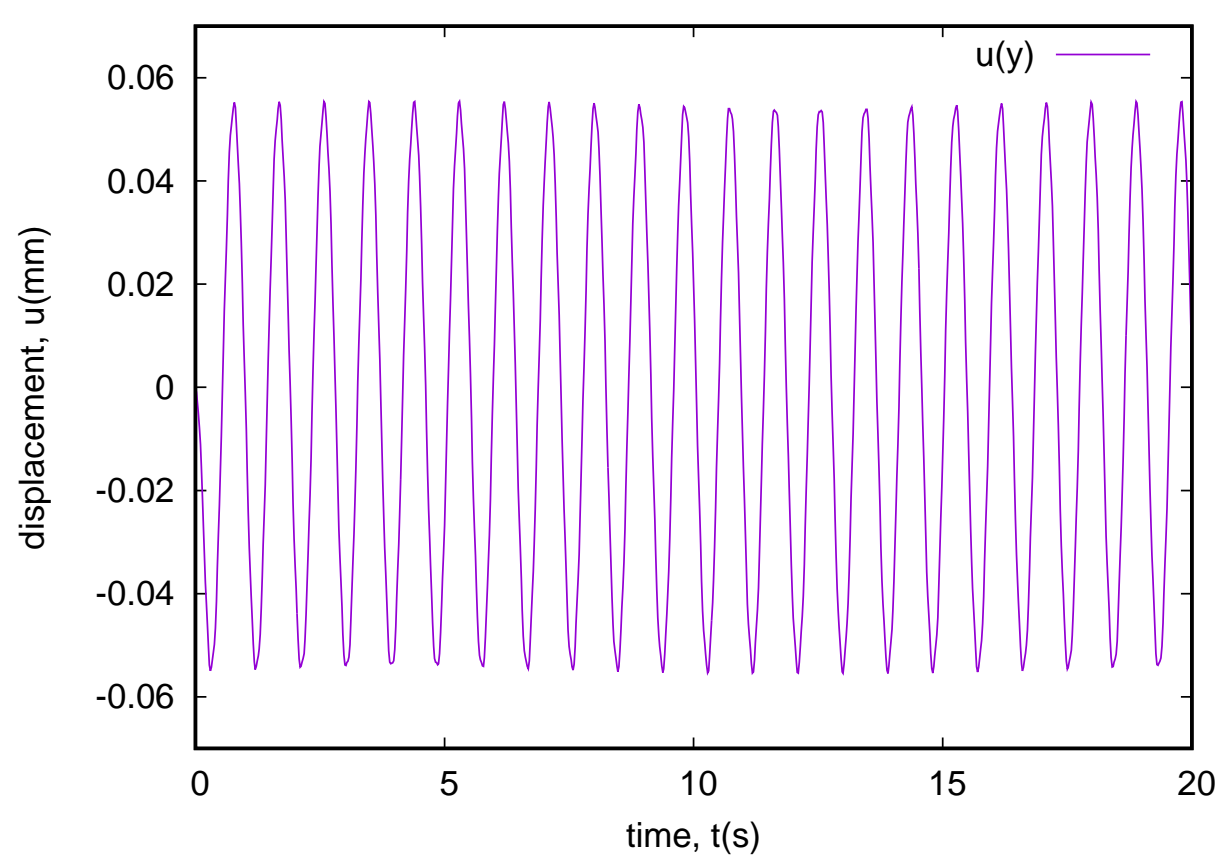

Figure 5.4: Displacement history of the mid-span of a simply supported pipe undergoing free vibration

We obtain $\omega_{n}=0.791$ with 20 finite elements and Figure5.6 below shows the free-end displacement of the pipe.

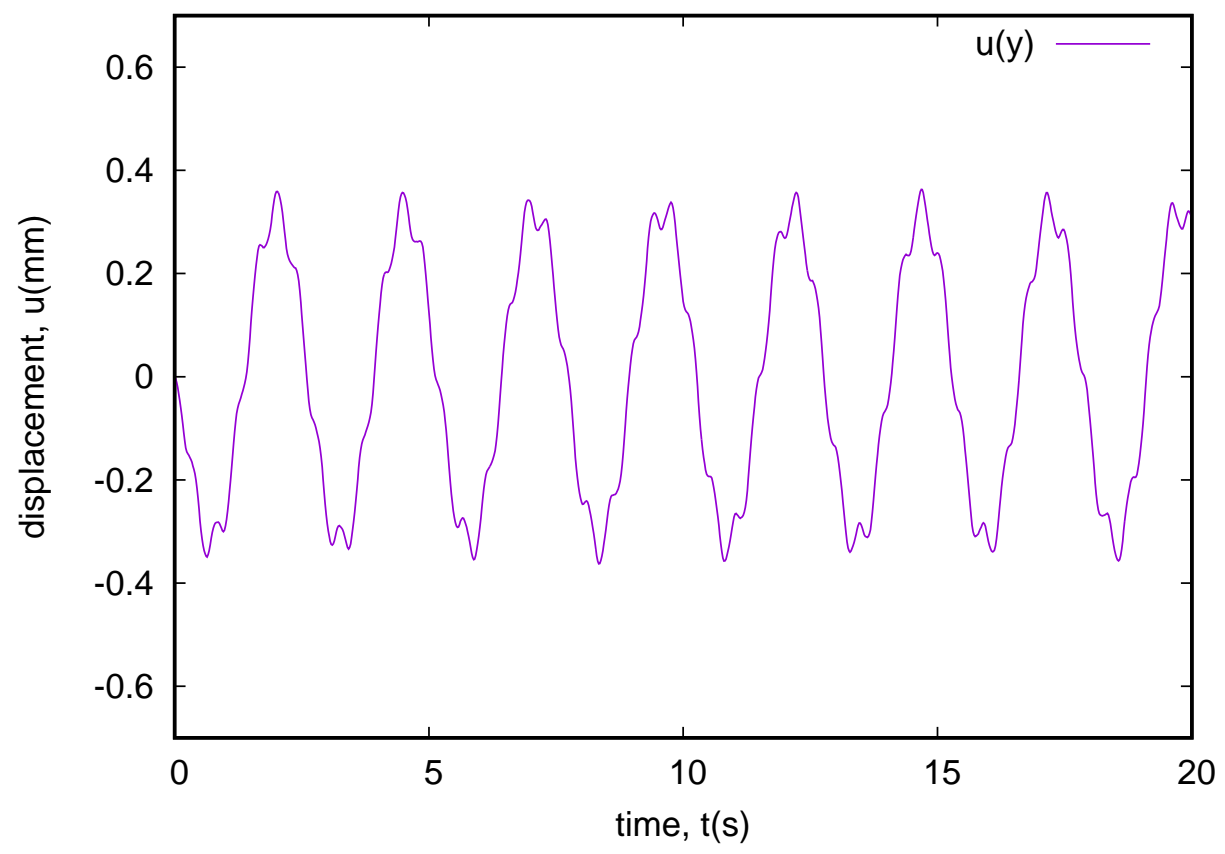

Figure 5.6: Displacement history of the free end of a cantilever pipe undergoing free vibration 


\subsubsection{Subcritical fluid flow through the pipe}

For small deformation of a simply supported pipe with Young's modulus $E=2.5 \times$ $10^{7} \mathrm{Nm}^{-2}$, all the other properties remain the same, conveying fluid with velocity $v<v_{c}$, we obtain very good agreement with the linear response of the system. Figure 5.8 below shows the mid-span displacement histories for velocities, $v=2.8 \mathrm{~ms}^{-1}$, and $v=7 \mathrm{~ms}^{-1}$ respectively, for both the linear and nonlinear problem, and we have excellent agreement with the linear case.

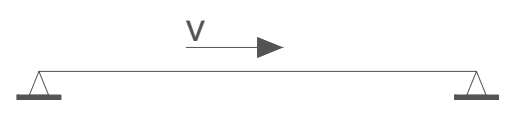

Figure 5.7: Geometry

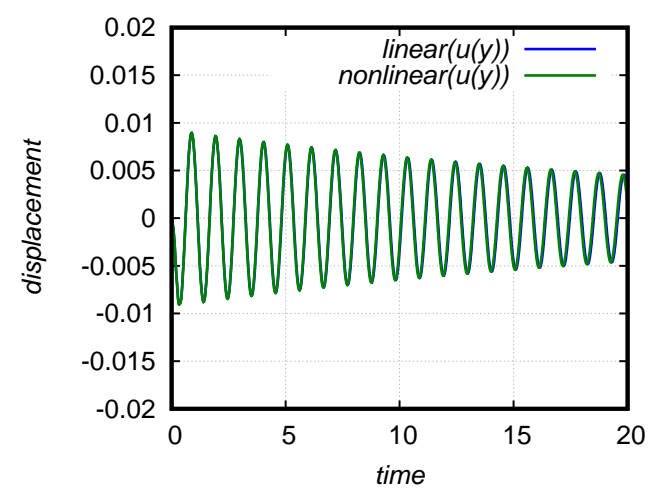

(a) $v=2.8$

$$
\begin{aligned}
& L=2 \mathrm{~m} \\
& E=2.5 \times 10^{7} \mathrm{Nm}^{-2} \\
& \text { pipe density } \rho_{p}=1167 \mathrm{kgm}^{-3} \\
& \rho_{f}=999 \mathrm{kgm}^{-3} \\
& d_{i}=32 \mathrm{~mm} \\
& d_{o}=70 \mathrm{~mm} \\
& \delta t=0.001 \mathrm{~s}
\end{aligned}
$$

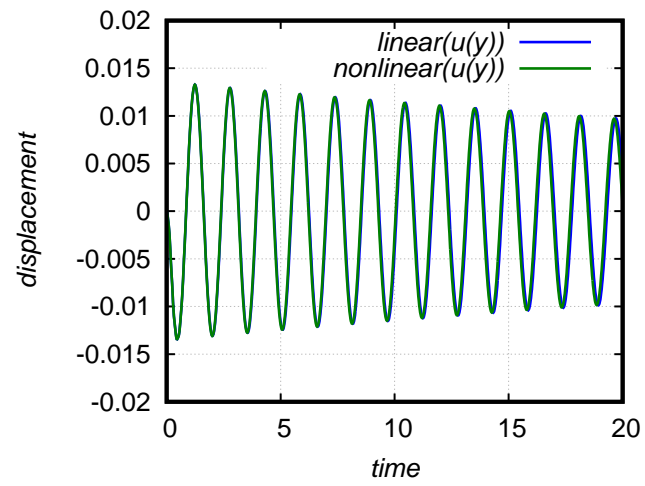

(b) $v=7$

Figure 5.8: Displacement history of the mid-span of a simply supported pipe for various flow velocities

Below is the current configuration for the simply supported pipe for $v=7$, at different time steps (Figure 5.9.

For velocity $v=7 \mathrm{~ms}^{-1}$, Table 5.1 shows the residuals for 2 time steps arbitrarily chosen so as not to always pick the last 2. Quadratic convergence is observed as expected.

A cantilever pipe is stable for flow below the critical velocity, given as a function 


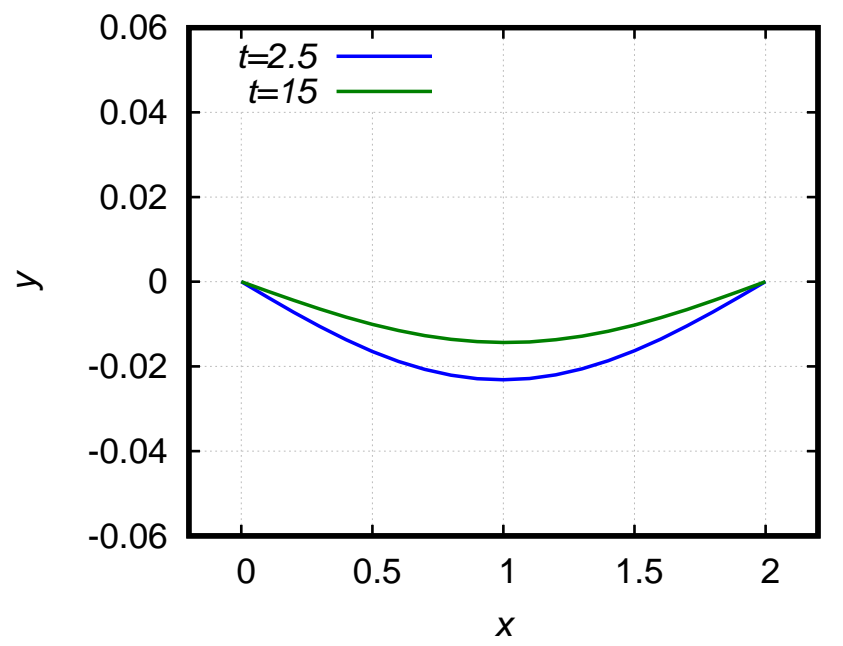

Figure 5.9: Current configuration for the simply supported pipe $(v=7)$

\begin{tabular}{c|cc}
\hline iteration & $t_{i}$ & $t_{j}$ \\
\hline 1 & $1.08 \times 10^{2}$ & $2.64 \times 10^{1}$ \\
2 & $9.865 \times 10^{4}$ & $9.7611 \times 10^{4}$ \\
3 & $1.12 \times 10^{1}$ & $1.1123 \times 10^{1}$ \\
4 & $3.128 \times 10^{-} 2$ & $9.9173 \times 10^{-} 1$ \\
5 & $2.156 \times 10^{-} 6$ & $4.95 \times 10^{-} 5$ \\
6 & $3.0388 \times 10^{-} 10$ & $5.6294 \times 10^{-} 9$ \\
\hline
\end{tabular}

Table 5.1: Convergence table for 2 arbitrarily chosen time steps for velocity, $7 \mathrm{~ms}^{-1}$

of mass ratio from [10] as

$$
\frac{\rho A}{M}=0.1844 \quad v_{c} L\left(\frac{\rho A}{E I}\right)^{\frac{1}{2}} \approx 5.5 \quad v_{c} \approx 16.285
$$

$$
\begin{array}{ll}
L=2 \mathrm{~m} \\
E=2.5 \times 10^{7} \mathrm{Nm}^{-2} \\
\text { pipe density } \rho_{p}=1167 \mathrm{kgm}^{-3} \\
\rho_{f}=999 \mathrm{kgm}^{-3} \\
\quad d_{i}=32 \mathrm{~mm} \\
\quad d_{o}=70 \mathrm{~mm} \\
\delta t=0.001 \mathrm{~s}
\end{array}
$$

Figure 5.10: Geometry

Displacement history for velocities $v=5.4$ and $v=9.2$ are compared against 
results for the linear response of the system and shown in Figure 5.11 below. Both the linear and nonlinear problem are shown on the same plot to show excellent agreement for the case. The residuals for $v=9.2$ is shown in Table 5.2 below

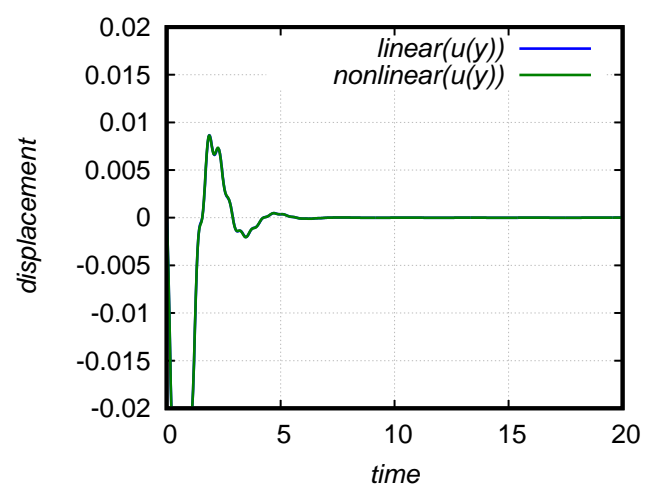

(a) $v=5.4$

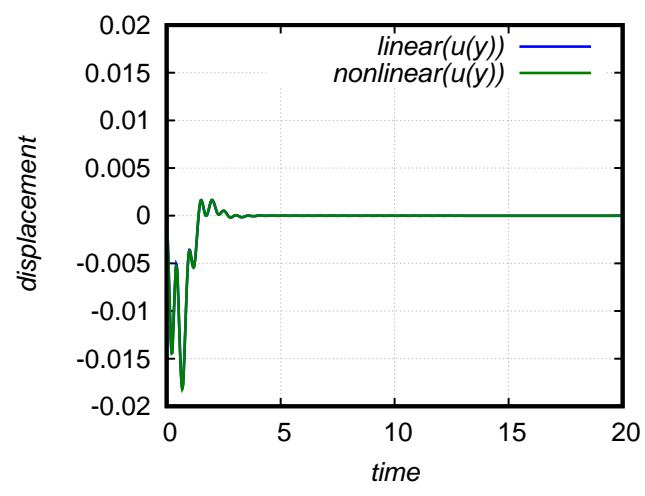

(b) $v=9.2$

Figure 5.11: Displacement history of the free end of a cantilever pipe for various flow velocities

\begin{tabular}{c|cc}
\hline iteration & $t_{i}$ & $t_{j}$ \\
\hline 1 & $3.211 \times 10^{4}$ & $4.5774 \times 10^{2}$ \\
2 & $7.125 \times 10^{2}$ & $6.118 \times 10^{-} 1$ \\
3 & $5.5569 \times 10^{1}$ & $3.95 \times 10^{-} 3$ \\
4 & $2.547 \times 10^{-} 1$ & $2.83 \times 10^{-} 7$ \\
5 & $1.18 \times 10^{-} 5$ & $8.492 \times 10^{-} 9$ \\
6 & $9.0466 \times 10^{-} 9$ & - \\
\hline
\end{tabular}

Table 5.2: Convergence table for 2 arbitrarily chosen time steps for velocity, $9.2 \mathrm{~ms}^{-1}$

\subsubsection{Instability of pipes}

A cantilever pipe conveying fluid with velocity greater than the critical velocity $\left(v>v_{c}\right)$ is presented here, and Figure 5.13 shows the vibration of the pipe for velocities $v=18$, and $v=20$ obtained with 20 finite elements. Figure 5.14 also shows the maximum amplitude of vibration of the pipe for various velocities. As expected, the pipe flails about with the amplitude of vibration increasing with the velocity, beyond the critical velocity. 


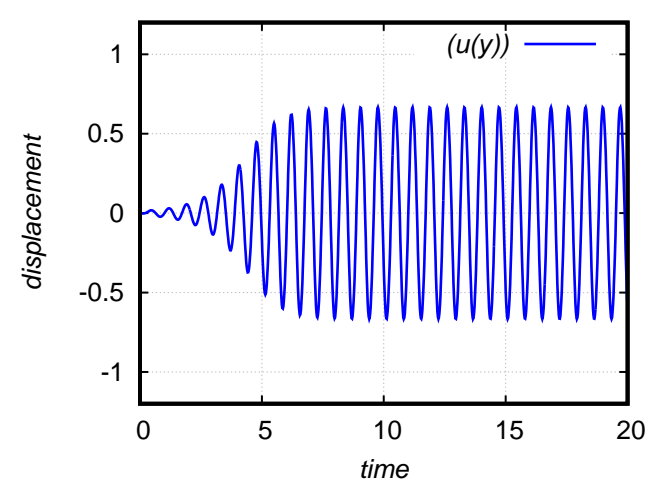

(a) $v=18$

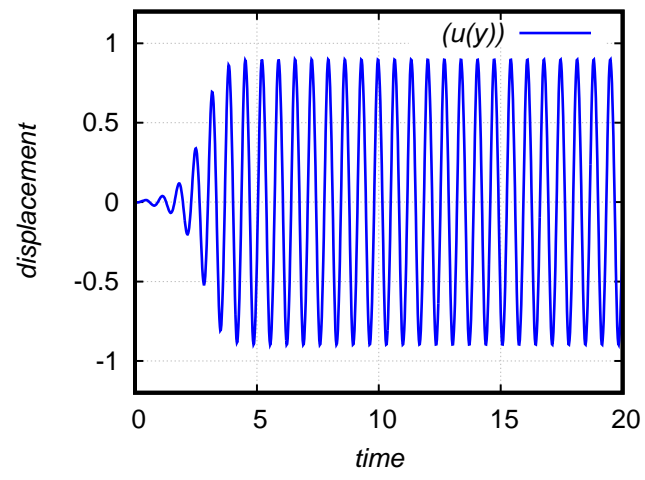

(b) $v=20$

Figure 5.13: Displacement history of the free end of a cantilever pipe for velocities beyond the critical velocity

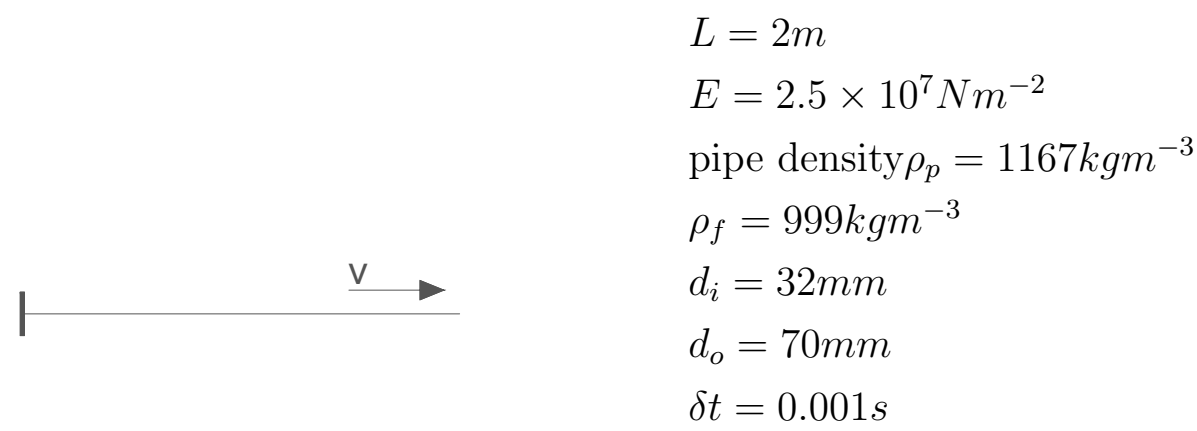

Figure 5.12: Geometry

Figure 5.15 shows the pipe as it flails about with maximum amplitude increasing as the velocity increases beyond the critical velocity.

For velocity $v=20 \mathrm{~ms}-1$ the residuals are shown in Table 5.3 below.

\begin{tabular}{c|cc}
\hline iteration & $t_{i}$ & $t_{j}$ \\
\hline 1 & $9.1943 \times 10^{1}$ & $6.0674 \times 10^{2}$ \\
2 & $2.5126 \times 10^{-} 1$ & $8.2151 \times 10^{3}$ \\
3 & $1.1371 \times 10^{-} 3$ & $9.341 \times 10^{-} 1$ \\
4 & $1.5042 \times 10^{-} 9$ & $4.06 \times 10^{-} 5$ \\
5 & - & $4.982 \times 10^{-} 11$ \\
\hline
\end{tabular}

Table 5.3: Convergence table for 2 arbitrarily chosen time steps for velocity, $20 \mathrm{~ms}^{-1}$

For a simply supported pipe on the other hand, the pipe buckles when fluid flows through with velocity $v>v_{c}$. From [10, the critical velocity is given as 


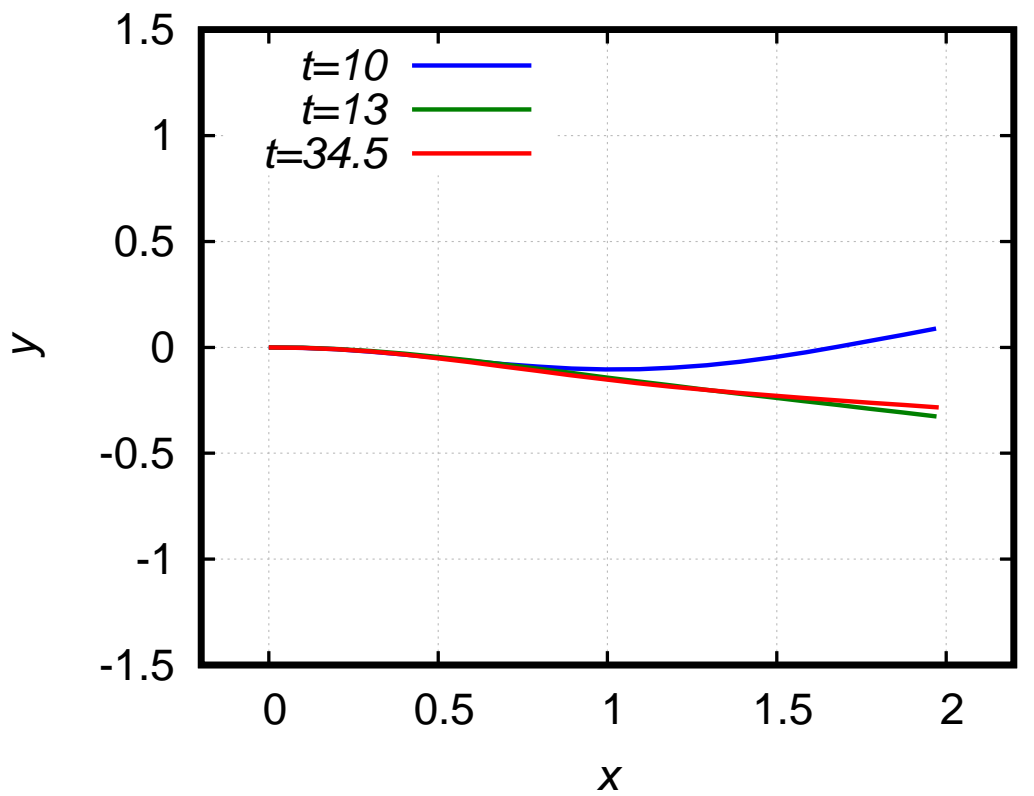

Figure 5.14: $v=16.9$

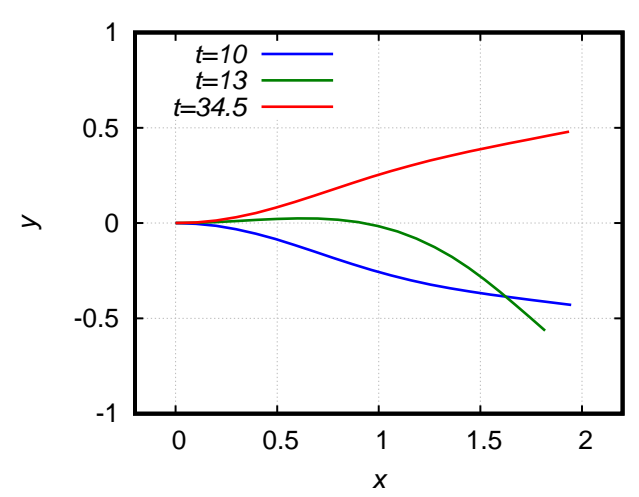

(a) $v=18$

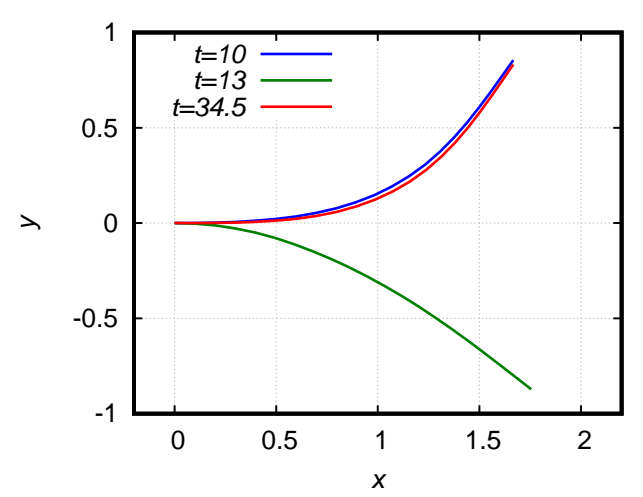

(b) $v=20$

Figure 5.15: Pipe configuration for flow with velocities beyond the critical velocity

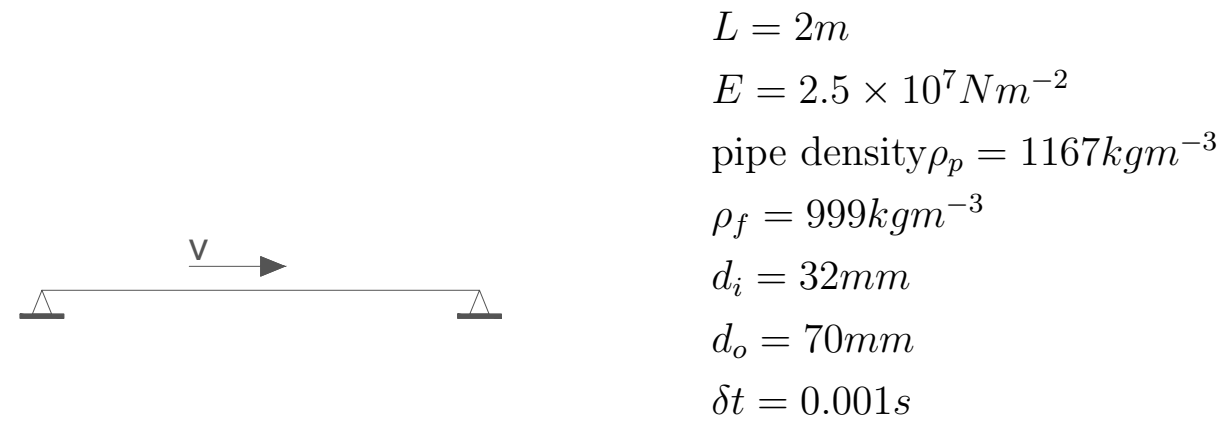

Figure 5.16: Geometry 


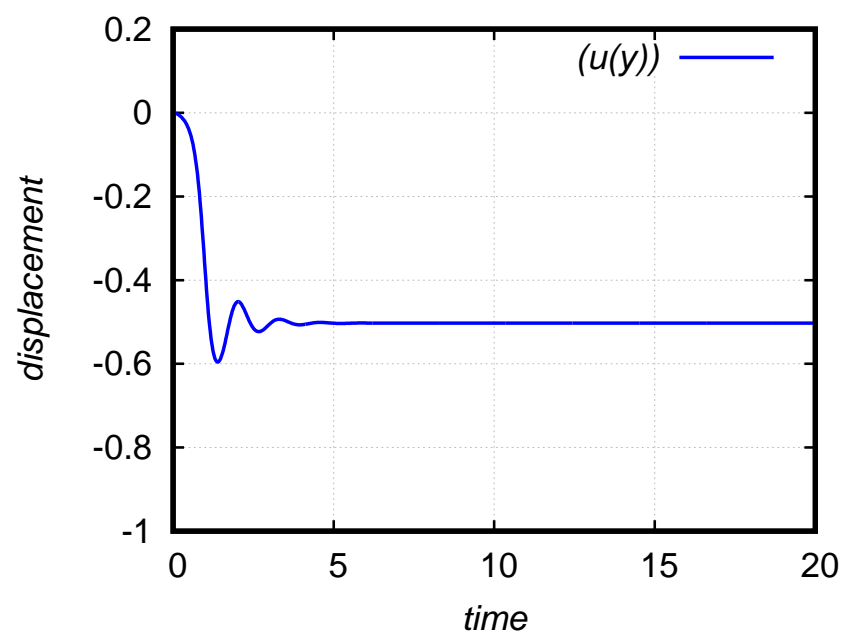

Figure 5.17: Buckling instability of a simply supported pipe with fluid flowing at a velocity above the critical velocity

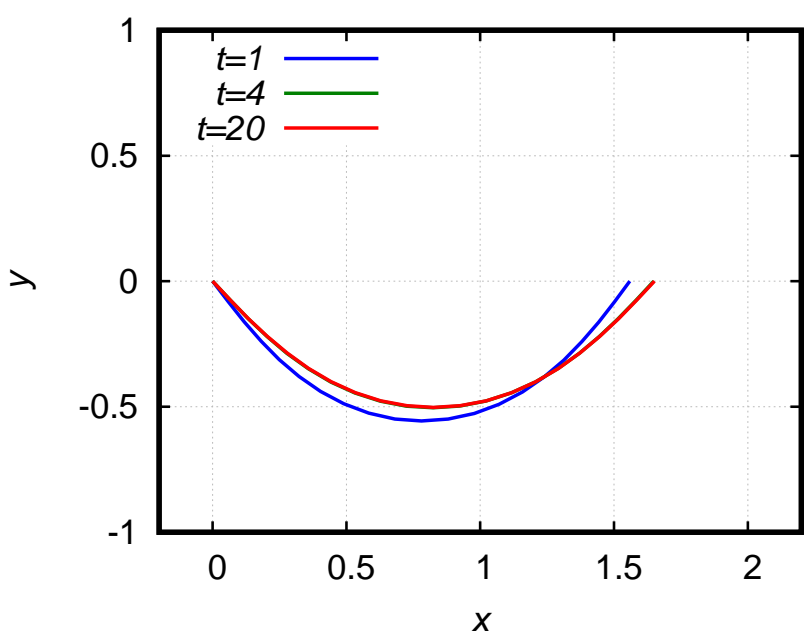

Figure 5.18: Instability of a simply supported pipe

$$
v_{c}=\frac{\pi}{L}\left(\frac{E I}{\rho A}\right)^{\frac{1}{2}}=9.3022
$$

Figure 5.17 shows the displacements history of the pipe with fluid flowing with velocity, $v=12$, Figure 5.18 shows the deformed configuration of the pipe, and Table 5.4 shows the residuals for $v=12$. 


\begin{tabular}{c|cc}
\hline iteration & $t_{i}$ & $t_{j}$ \\
\hline 1 & $5.012 \times 10^{1}$ & $8.225 \times 10^{1}$ \\
2 & $6.2415 \times 10^{-} 2$ & $4.547 \times 10^{-} 2$ \\
3 & $2.39 \times 10^{-} 5$ & $7.1002 \times 10^{-} 5$ \\
4 & $1.042 \times 10^{-} 8$ & $4.58 \times 10^{-} 9$ \\
\hline
\end{tabular}

Table 5.4: Convergence table for 2 arbitrarily chosen time steps for velocity, $12 \mathrm{~ms}^{-1}$

\subsection{Conclusion}

Serving as a bridge between the two-dimensional linear examples presented in a previous section of this work, and the three-dimensional nonlinear investigation which is still to come, the simulations presented in this chapter agreed with all results to which they were compared against, primarily available analytical solutions, and also to the results of the simulations form the linear case, where applicable (for small deformation). Matching analytical solutions and results of the linear simulations which they were expected to (critical velocities and frequencies of vibration in particular), while displaying expected nonlinear behaviour will be very useful. Before moving on to explore the kaleidoscope of interesting behaviour the three-dimensional systems promise to deliver, they will first be matched against these results to demonstrate the accuracy of the method, and ensure confidence what they have to offer. 


\section{Chapter 6}

\section{Pipes Conveying fluid: 3D nonlinear solution}

\subsection{Introduction}

This chapter brings the work together by presenting the 3D Finite element solution of non-linear problems, in the same manner as the previous chapters. The various components of the equation of motion are put together, and the numerical examples show the ability of the element to simulate 3D pipes conveying fluid, as well as accuracy of the results

\subsection{Finite element approximation}

\subsubsection{Pipe stiffness term}

In a similar manner to the treatment of the 2dimensional nonlinear solution, the various terms in the equation of motion are handled separately and their contributions are put together afterwards and assembled in the usual manner. First off is the pipe stiffness term which has been treated extensively in chapter 2 of this work. The important components for this section are the contributions to the residual from Equation 2.5.7, and upon application of the directional derivative, we obtain the tangent stiffness matrix. 


\subsubsection{Centrifugal force term}

The second term is the centrifugal force contribution, and we have

$$
f_{c}=\rho A v^{2} \frac{\delta}{\delta s^{2}}\left\{\begin{array}{l}
x \\
y \\
z
\end{array}\right\}
$$

Applying the principle of virtual work and integrating by parts, we have for an element

$$
\begin{gathered}
\int_{e} \delta y \rho A v^{2} y^{\prime \prime} d s=\rho A v^{2} \int_{e} \delta y y^{\prime \prime} d s \\
=\rho A v^{2}\left(\left[\delta y^{\prime}\right]_{0}^{l}-\int_{e} \delta y^{\prime} y^{\prime} d s\right)
\end{gathered}
$$

Here, $y$ represents the vector of degrees of freedom and $\delta y$, the admissible virtual counterparts.

Introduced in the previous section,

$$
\frac{\delta x}{\delta s}=\frac{\Delta x}{l}, \quad \frac{\delta y}{\delta s}=\frac{\Delta y}{l}, \quad \text { and } \quad \frac{\delta z}{\delta s}=\frac{\Delta z}{l}
$$

the contribution of the second term in Equation 6.2.3 is added to all elements of the pipe, and the first term is applied at the ends of the pipe, hence the first and last element.

Using a 2-node linear finite element we have the shape functions

$$
\begin{aligned}
& N_{1}=1-x / l \\
& N_{2}=x / l
\end{aligned}
$$

From 6.2.3, 6.2.4, 6.2.5, we have for the second term

$$
[-\Delta x,-\Delta y,-\Delta z, 0,0,0, \Delta x, \Delta y, \Delta z, 0,0,0]^{T} \cdot \frac{\rho A v^{2}}{l}
$$

which is premultiplied by -1 and added to all elements of the pipe, following from 6.2 .3 .

Similarly, for the first term, we have

$$
[-\Delta x,-\Delta y,-\Delta z, 0,0,0,0,0,0,0,0,0]^{T} \cdot \frac{\rho A v^{2}}{l}
$$


to the first element, and

$$
[0,0,0,0,0,0, \Delta x, \Delta y, \Delta z, 0,0,0]^{T} \cdot \frac{\rho A v^{2}}{l}
$$

to the last element

The element tangent matrix is obtained by employing the directional derivative to obtain

$$
K_{c}=\left[\begin{array}{cccc}
\alpha_{3 x 3} & \ldots & -\alpha_{3 x 3} & \ldots \\
\vdots & \ddots & \vdots & \\
-\alpha_{3 x 3} & \ldots & \alpha_{3 x 3} & \ldots \\
\vdots & \vdots & \vdots & \vdots
\end{array}\right]
$$

with

$$
\alpha=\left[\begin{array}{lll}
\alpha_{1} & \alpha_{2} & \alpha_{4} \\
\alpha_{2} & \alpha_{3} & \alpha_{5} \\
\alpha_{4} & \alpha_{5} & \alpha_{6}
\end{array}\right]
$$

Where

$$
\begin{aligned}
& \alpha_{1}=\frac{\Delta x^{2}}{l^{3}}-\frac{1}{l} \\
& \alpha_{2}=\frac{\Delta x \Delta y}{l^{3}} \\
& \alpha_{3}=\frac{\Delta y^{2}}{l^{3}}-\frac{1}{l} \\
& \alpha_{4}=\frac{\Delta x \Delta z}{l^{3}} \\
& \alpha_{5}=\frac{\Delta y \Delta z}{l^{3}} \\
& \alpha_{6}=\frac{\Delta z^{2}}{l^{3}}-\frac{1}{l}
\end{aligned}
$$

The top half of $K_{c}$ is subtracted from the first element, and the bottom half is subtracted from the last element 


\subsubsection{Coriolis force term}

For the Coriolis term, we have

$$
f_{r}=2 \rho A v \frac{\delta}{\delta s \delta t}\left\{\begin{array}{l}
x \\
y \\
z
\end{array}\right\}
$$

Introducing the finite element approximation and using 2-noded linear elements, we have

$$
\int_{e} 2 \rho A v \frac{\delta}{\delta t}\left\{\begin{array}{l}
\frac{\delta x}{\delta s} \\
\frac{\delta y}{\delta s} \\
\frac{\delta z}{\delta s}
\end{array}\right\} \cdot\left\{\begin{array}{l}
\delta x \\
\delta y \\
\delta z
\end{array}\right\}
$$

Simplifying the above expression results in

$$
F_{r}^{e}=\rho A v l \cdot \frac{\delta}{\delta t}\left[\frac{\Delta x}{l}, \frac{\Delta y}{l}, \frac{\Delta z}{l}, 0,0,0, \frac{\Delta x}{l}, \frac{\Delta y}{l}, \frac{\Delta z}{l}, 0,0,0\right]^{T}
$$

Using $\alpha_{i}$ above, and defining the following,

$$
\begin{aligned}
\gamma_{1} & =\frac{\delta}{\delta t} \cdot \frac{\delta x}{l} \\
\gamma_{2} & =\frac{\delta}{\delta t} \cdot \frac{\delta y}{l} \\
\gamma_{3} & =\frac{\delta}{\delta t} \cdot \frac{\delta z}{l}
\end{aligned}
$$

and 


$$
\begin{aligned}
& \beta_{1}=\alpha_{1} \cdot \frac{l}{2 \Delta t} \\
& \beta_{2}=\alpha_{2} \cdot \frac{l}{2 \Delta t} \\
& \beta_{3}=\alpha_{3} \cdot \frac{l}{2 \Delta t} \\
& \beta_{4}=\alpha_{4} \cdot \frac{l}{2 \Delta t} \\
& \beta_{5}=\alpha_{5} \cdot \frac{l}{2 \Delta t} \\
& \beta_{6}=\alpha_{6} \cdot \frac{l}{2 \Delta t} \\
& \beta_{7}=\frac{\Delta x}{l} \cdot \rho A v \\
& \beta_{8}=\frac{\Delta y}{l} \cdot \rho A v \\
& \beta_{9}=\frac{\Delta z}{l} \cdot \rho A v
\end{aligned}
$$

The element tangent matrix is obtained by employing the directional derivative to obtain

$$
K_{f}=\left[\begin{array}{cccc}
\beta_{3 x 3} & \ldots & -\beta_{3 x 3} & \ldots \\
\vdots & \ddots & \vdots & \\
-\beta_{3 x 3} & \ldots & \beta_{3 x 3} & \ldots \\
\vdots & \vdots & \vdots & \vdots
\end{array}\right]
$$

with

$$
\beta=\left[\begin{array}{lll}
\beta_{1}-\gamma_{1} \cdot \beta_{7} & \beta_{2}-\gamma_{1} \cdot \beta_{8} & \beta_{4}-\gamma_{1} \cdot \beta_{9} \\
\beta_{2}-\gamma_{2} \cdot \beta_{7} & \beta_{3}-\gamma_{2} \cdot \beta_{8} & \beta_{5}-\gamma_{2} \cdot \beta_{9} \\
\beta_{4}-\gamma_{3} \cdot \beta_{7} & \beta_{5}-\gamma_{3} \cdot \beta_{8} & \beta_{6}-\gamma_{3} \cdot \beta_{9}
\end{array}\right]
$$

\subsubsection{Inertia term}

For the inertia term, we have

$$
\int_{0}^{l}(m+\rho A)(s) \ddot{y}(s, t) \delta y(s) d s
$$

Simplifying, we obtain the consistent mass matrix as

$$
\boldsymbol{M}^{e}=\int_{0}^{l^{e}}(m+\rho A) N_{1} N_{2} d s
$$


which yields

$$
\boldsymbol{M}^{e}=\frac{(m+\rho A) l}{6}\left[\begin{array}{cccccccccccc}
2 & 0 & 0 & 0 & 0 & 0 & 1 & 0 & 0 & 0 & 0 & 0 \\
0 & 2 & 0 & 0 & 0 & 0 & 0 & 1 & 0 & 0 & 0 & 0 \\
0 & 0 & 2 & 0 & 0 & 0 & 0 & 0 & 1 & 0 & 0 & 0 \\
0 & 0 & 0 & 0 & 0 & 0 & 0 & 0 & 0 & 0 & 0 & 0 \\
0 & 0 & 0 & 0 & 0 & 0 & 0 & 0 & 0 & 0 & 0 & 0 \\
0 & 0 & 0 & 0 & 0 & 0 & 0 & 0 & 0 & 0 & 0 & 0 \\
1 & 0 & 0 & 0 & 0 & 0 & 2 & 0 & 0 & 0 & 0 & 0 \\
0 & 1 & 0 & 0 & 0 & 0 & 0 & 2 & 0 & 0 & 0 & 0 \\
0 & 0 & 1 & 0 & 0 & 0 & 0 & 0 & 2 & 0 & 0 & 0 \\
0 & 0 & 0 & 0 & 0 & 0 & 0 & 0 & 0 & 0 & 0 & 0 \\
0 & 0 & 0 & 0 & 0 & 0 & 0 & 0 & 0 & 0 & 0 & 0 \\
0 & 0 & 0 & 0 & 0 & 0 & 0 & 0 & 0 & 0 & 0 & 0
\end{array}\right]
$$

Using the same time stepping scheme introduced in Section 3.4, the contributions from the various terms above are put together to obtain the global element force vector and the global element tangent operator. The assembly of elements in the same manner results in the global system of equations which is solved for the required degrees of freedom at the nodes using the Newton-Raphson solution procedure presented in previous chapters of this work.

\subsection{Numerical examples}

The determination of the critical velocity, beyond which we get flutter in cantilever systems, or buckling in simply supported systems due to loss of initial static stability is the starting point for our numerical investigation.

\subsubsection{Instability of cantilever pipes}

In the previous section, we presented a cantilever pipe with inner and outer diameter $d_{i}=32 \mathrm{~mm}$ and $d_{o}=70 \mathrm{~mm}$ respectively. With length $L=2 \mathrm{~m}$, Young's Modulus $E=2.5 \times 10^{7}$ and pipe density $\rho_{p}=1167$, the critical velocity was determined to be $v_{c} \approx 16.285$ (See Equation 5.4.3), beyond which the cantilever flailed about with increasing amplitude as the velocity increased. For the three-dimensional solution, we also obtain good agreement with the analytical results, recording $v_{c} \approx 16.5$ as the critical velocity. Displacement history of the free end is shown in Figure 6.2 which matches those obtained from the two-dimensional nonlinear simulations in 


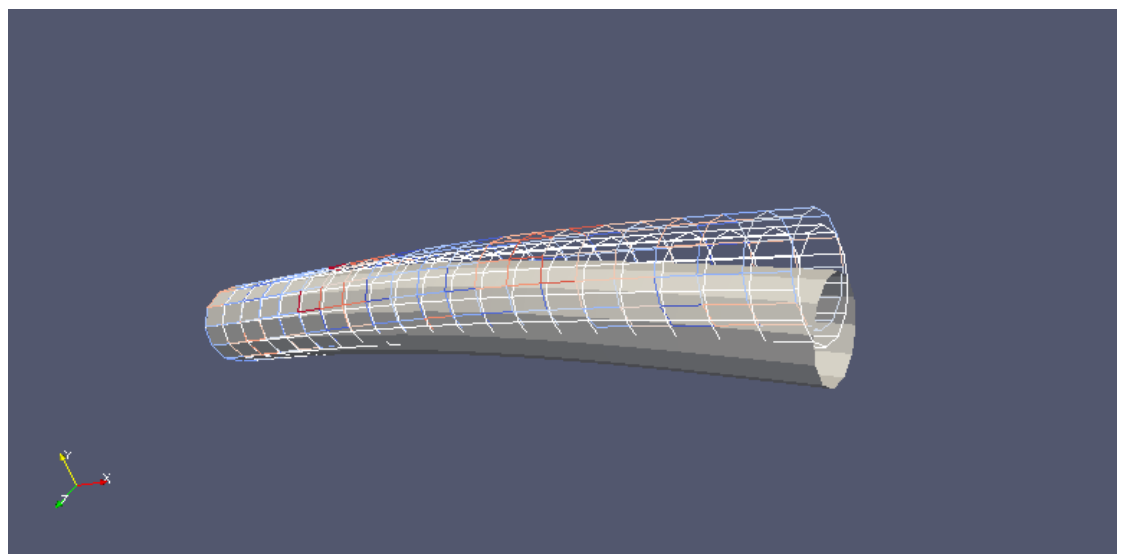

Figure 6.1: Initial and deformed cantilever pipe $v=9.2$

the previous chapter, and the results are so close that there appears to be only 1 line in the plot

Beyond the critical velocity, we get expected flutter, and Figure 6.3 and 6.4 provide information on the deformation. Figure 6.3 shows the pipe at various stages of the motion for visualisation purposes, and Figure 6.4 compares the 2D and 3D simulations. Unlike with the previous example where there are slight differences in certain sections of the plot, the two are practically identical in this case. Finally, with the right imperfection along the pipe, we get a full three-dimensional flutter of the pipe shown in Figure 6.5

The residuals for $v=20 \mathrm{~ms}^{-1}$ are presented in Table 6.1 to show quadratic convergence, as expected of the Newton-Raphson solution procedure.

\begin{tabular}{c|cc}
\hline iteration & $t_{i}$ & $t_{j}$ \\
\hline 1 & $5.12 \times 10^{3}$ & $5.0477 \times 10^{3}$ \\
2 & $3.1 \times 10^{1}$ & $0.1855 \times 10^{1}$ \\
3 & $1.8754 \times 10^{-} 1$ & $0.475 \times 10^{-} 2$ \\
4 & $4.25 \times 10^{-} 3$ & $0.22 \times 10^{-} 4$ \\
5 & $0.3991 \times 10^{-} 8$ & $2.004 \times 10^{-} 7$ \\
\hline
\end{tabular}

Table 6.1: Table of residuals for $\mathrm{v}=20$

\subsubsection{Simply Supported pipes}

Similarly for the simply supported pipe, as was the case with the cantilever problem, the result matches the two-dimensional nonlinear response of the system as shown in Figures 6.6, 6.7, 6.8, 6.9 below. The system is stable for velocities below the critical velocity $\left(v_{c}=9.3022\right)$, with frequency of vibration decreasing as we approach the critical velocity, where the pipe buckles. 


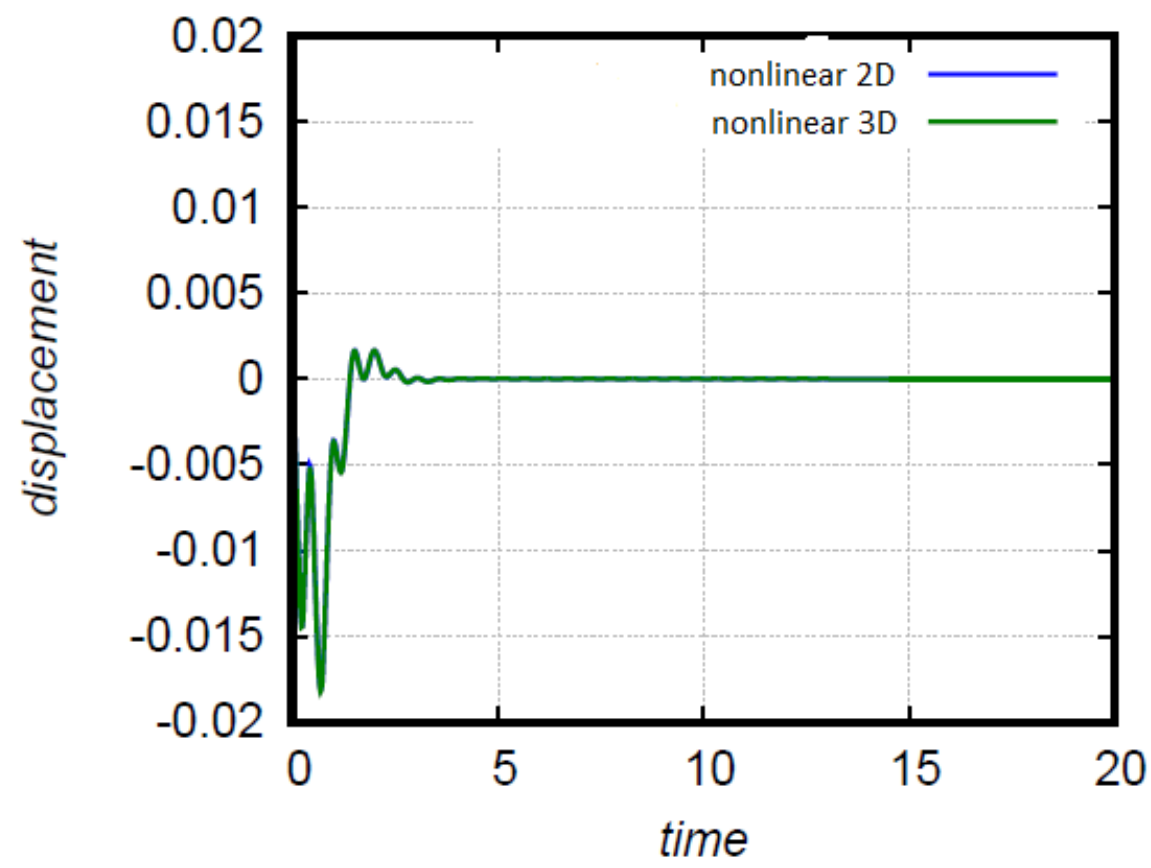

Figure 6.2: Comparison of free-end displacement history for $v=9.2$, for $2 \mathrm{D}$ and 3D

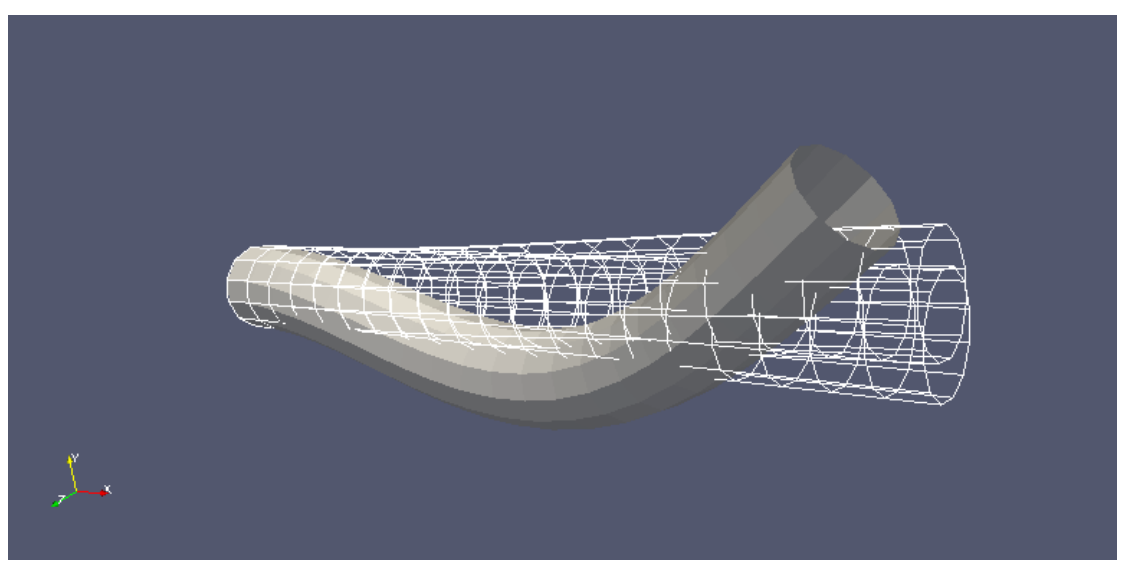

Figure 6.3: Initial and deformed cantilever pipe $v=20$ 


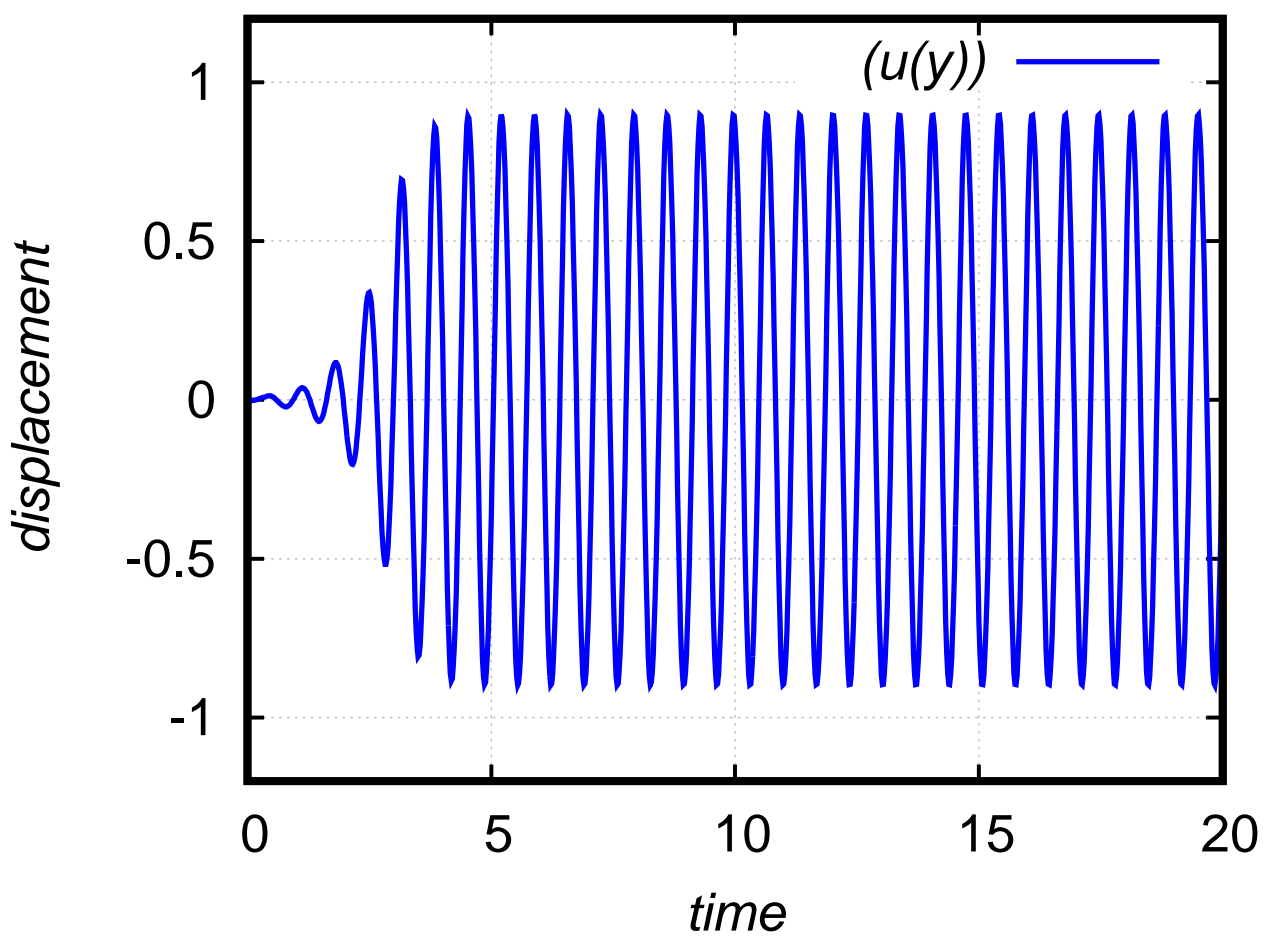

Figure 6.4: Comparison of free-end displacement history for $v=20$

For the simply supported case, with velocity $v=12$ the convergence table is shown below in Table 6.2

\begin{tabular}{c|cc}
\hline iteration & $t_{n-1}$ & $t_{n}$ \\
\hline 1 & $2.257 \times 10^{3}$ & $2.33 \times 10^{3}$ \\
2 & $7.8554 \times 10^{3}$ & $6.478 \times 10^{1}$ \\
3 & $0.1247 \times 10^{2}$ & $1.51 \times 10^{1}$ \\
4 & $9.21 \times 10^{1}$ & $8.25 \times 10^{-} 1$ \\
5 & $4.258 \times 10^{-} 2$ & $3.177 \times 10^{-} 2$ \\
6 & $1.11 \times 10^{-} 7$ & $0.9173 \times 10^{-} 6$ \\
7 & $0.77 \times 10^{-} 9$ & $1.2236 \times 10^{-} 9$ \\
\hline
\end{tabular}

Table 6.2: Table of residuals for $\mathrm{v}=12$

\subsection{Conclusion}

The numerical examples presented here agree with expected results, primarily the critical velocity, matching analytical results computed in previous chapters, and to which simulations of the 2D linear and nonlinear problems have already been compared against. Below the critical velocity, the cantilever pipe eventually stops 


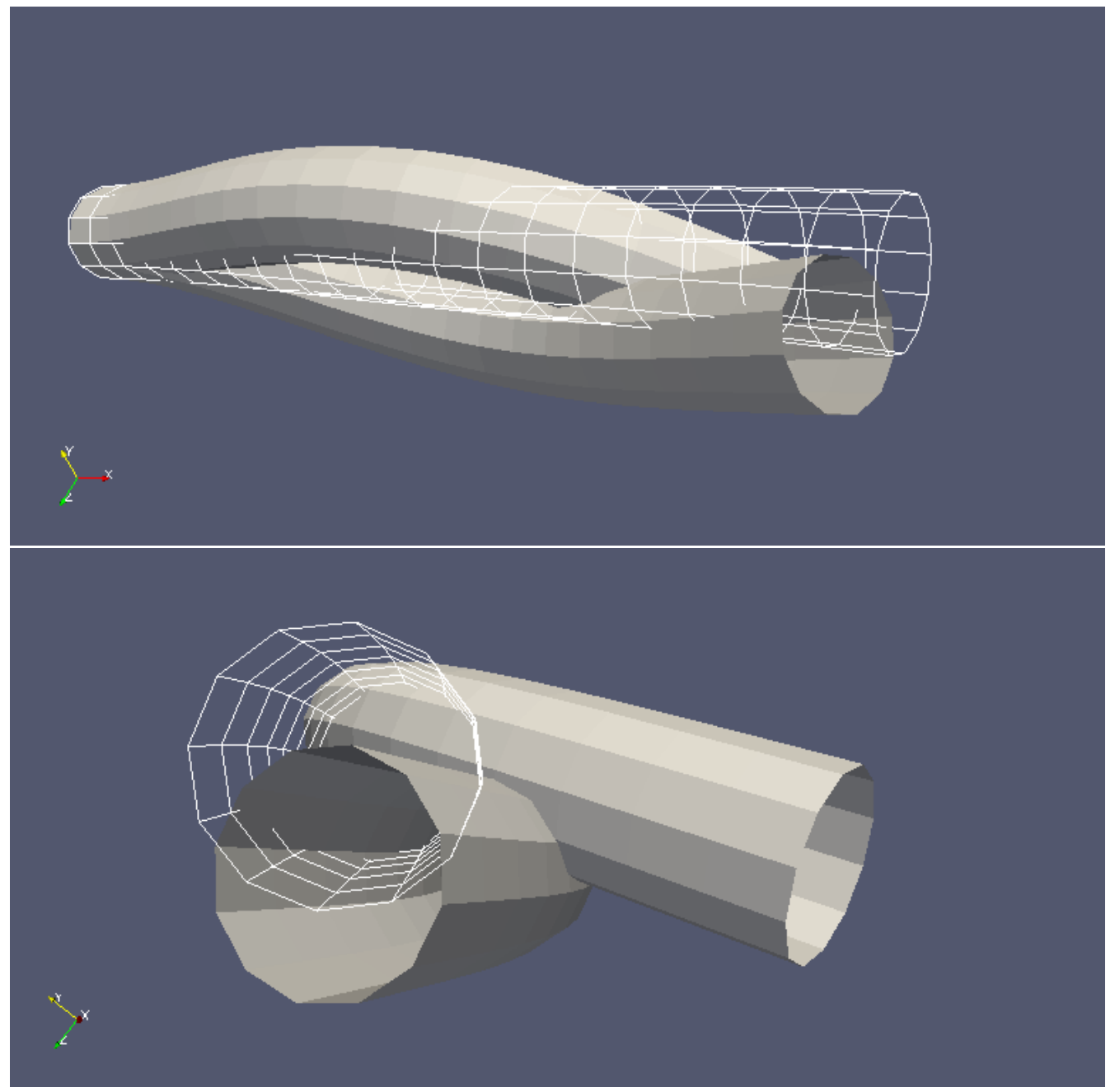

Figure 6.5: Chaotic oscillations

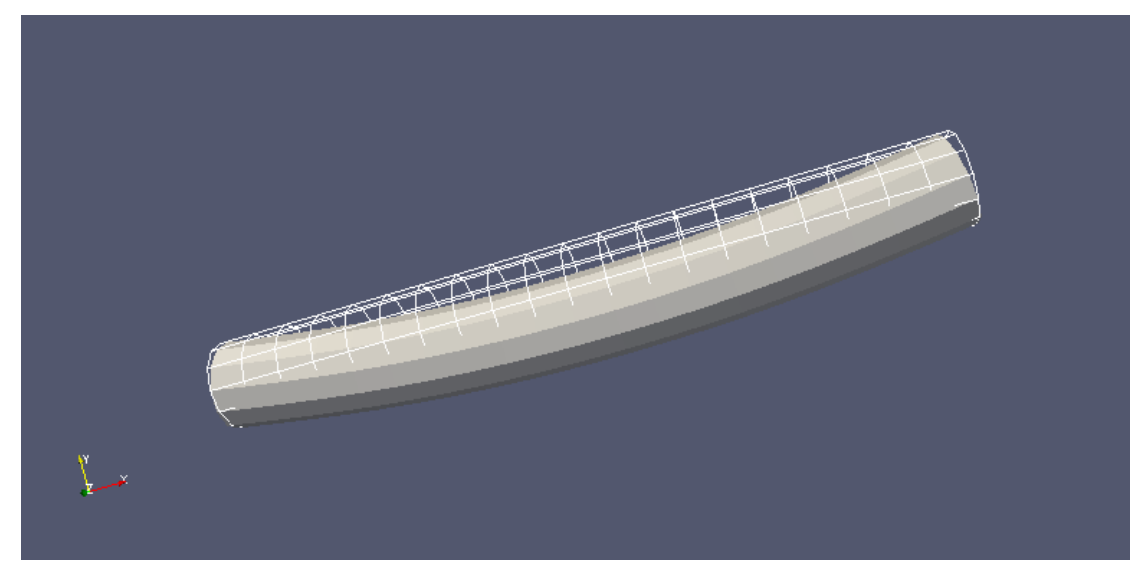

Figure 6.6: Initial and deformed cantilever pipe $v=7$ 


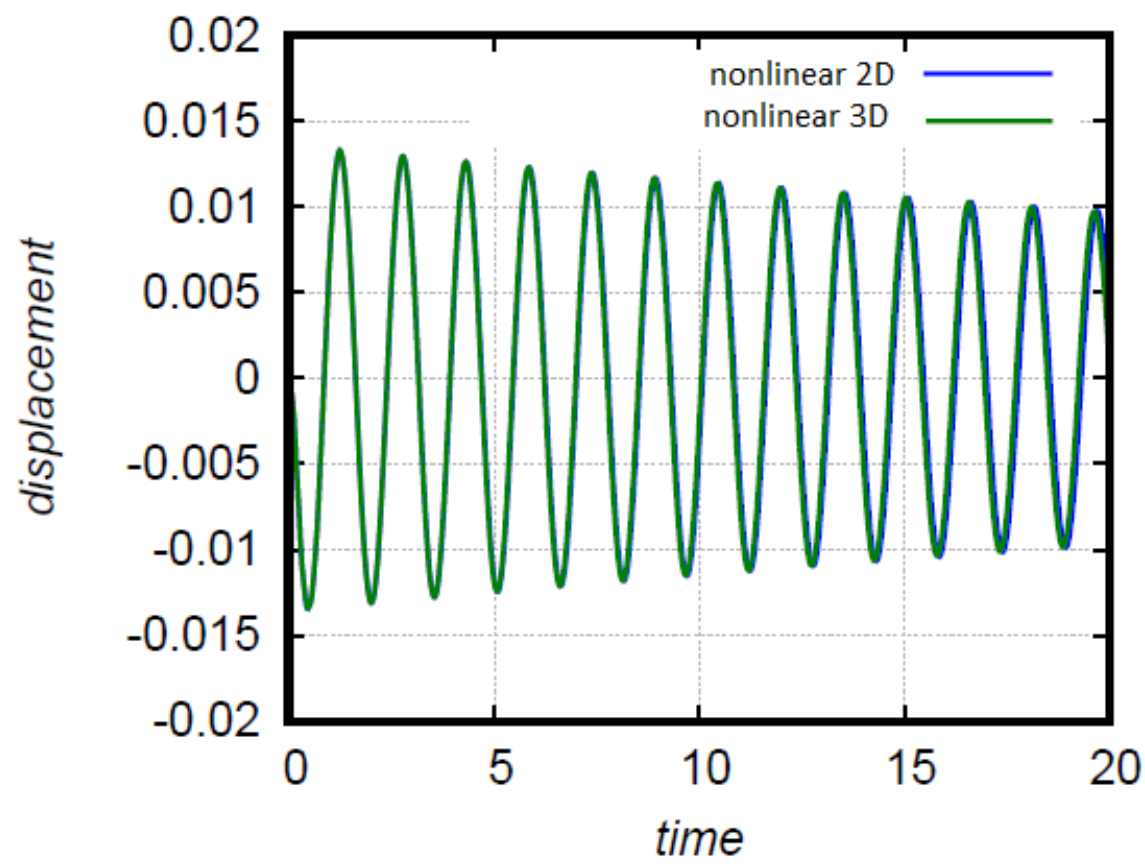

Figure 6.7: Comparison of free-end displacement history for $v=7$

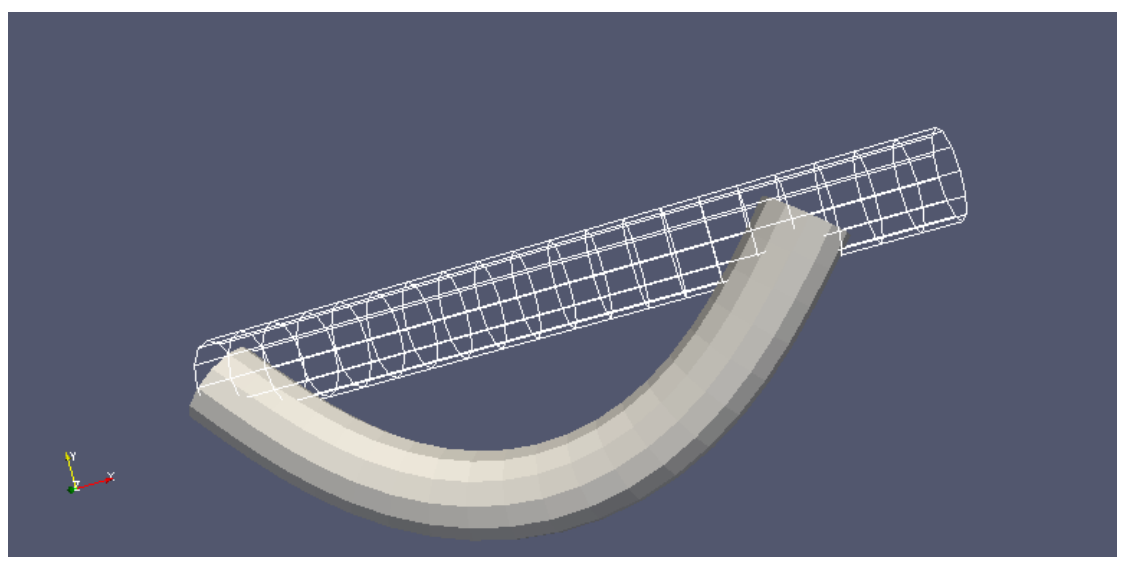

Figure 6.8: Initial and deformed cantilever pipe $v=12$

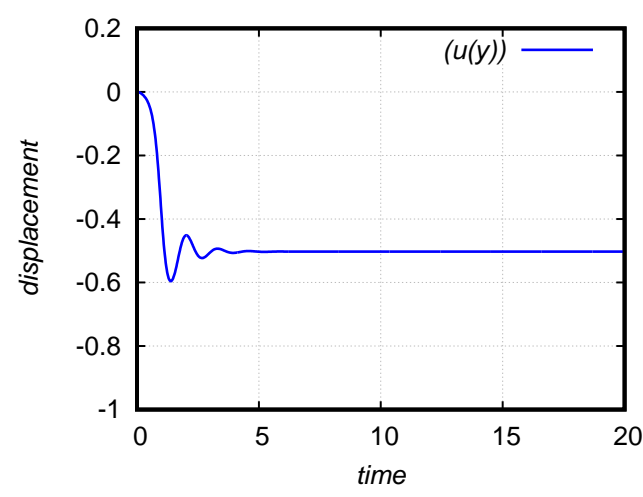

(a) $2 \mathrm{D}$

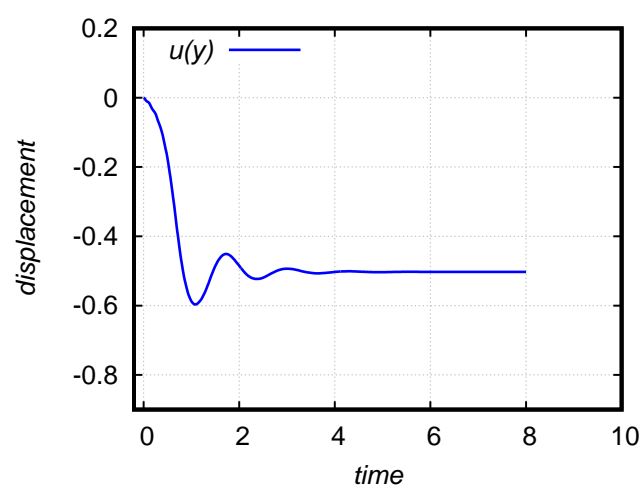

(b) $3 \mathrm{D}$

Figure 6.9: Comparison of free-end displacement history for $v=12$ 
vibrating, and the free end displacement history was compared against that of the $2 \mathrm{D}$ case, showing good agreement. A similar behaviour is observed in the simply supported case as the system is stable for values of velocity below the critical, and a comparison of the $3 \mathrm{D}$ result with the $2 \mathrm{D}$ problem showed good agreement. For flutter and buckling of the cantilever and simply supported pipes respectively, the amplitude and frequency of vibration of the cantilever for the 3D case was the same as its 2D counterpart, and for the buckling of the simply supported pipe, the final value for the deflection of the mid span of the pipe, after it comes to rest for both cases were the same.Chaotic oscillations of the pipe were also observed. The next chapter brings the work to a close, and presents a couple of suggestions for future work. 


\section{Chapter 7}

\section{Conclusion}

The aims if this thesis as outlined in section 1.2 have been achieved. Namely, a model for a flexible beam structure conveying fluid has been formulated and implemented. Based on the finite element method, interesting dynamic behaviour of various systems and problems were observed and validated against examples available in literature, some against analytical results, some compared against results obtained by other researchers, and some against other simulations presented in this work, as is the case with some $3 \mathrm{D}$ and $2 \mathrm{D}$ nonlinear problems. The nonlinear system of equations was solved using the Newton-Raphson solution procedure with quadratic convergence obtained, and the accuracy of the overall strategy was demonstrated through various numerical examples at various stages of the work.

The following describes the achievements of the work, and the thesis closes with recommendations for further research.

Primarily, the fully implicit version of the 3D geometrically exact beam element including an update of large rotations based on the Rodrigues formula was successfully implemented. Governing equations and solution procedure was shown in the work and various examples were presented to assess the performance of the model, showing excellent correspondence with results available in literature.

Additionally, in order to study the main features of MPAP2, further develop programming skills in $\mathrm{C}++$, and aid in validation of results, various beam and truss models in 2D and 3D were implemented. Numerical results from simulations matched available analytical solutions.

Next, the linear solution of the governing equation for pipes conveying fluid in 2D was presented. The generalized mid-point rule was used to solve the time-dependent problem, and numerical results including natural frequency of vibration, critical velocities, and the expected reduction in fundamental frequency were obtained, and matched analytical results for cantilevered and simply supported pipes. 
To obtain results for large displacements, as well as explore response of 2D systems beyond the critical velocity, a nonlinear solution was presented and results compared against the linear case for small deformations, showing good agreement. Fundamental frequency of vibration matched the analytical results, and flutter of cantilevered pipes and buckling of simply supported pipes was observed.

In $3 \mathrm{D}$, the equations of motion were presented, results matched problems studied in the literature and more interesting dynamic behaviour was observed as expected.

\subsection{Suggestions for future research}

The following are suggestions for future research which will build upon current achievements.

- To enable the simulation of a wider range of realistic problems, it is clearly necessary to extend the governing equations to chaotic dynamics as current applications are limited by initial assumptions made.

- Refining the fluid dynamic model would open up potential applications and problems which would otherwise not be considered.

- Problems of pipes conveying fluid immersed in fluid flow such as risers and underwater pipelines can also be studied, as preliminary investigation of immersed boundary methods showed promise. 


\section{Bibliography}

[1] J. Argyris. An excursion into large rotations. Computer Methods in Applied Mechanics and Engineering, 32(1-3):85-155, sep 1982.

[2] J. Argyris and F. Poterasu. Large rotations revisited application of Lie algebra. Computer Methods in Applied Mechanics and Engineering, 103(1-2):11-42, mar 1993.

[3] S. N. Atluri and A. Cazzani. Rotations in computational solid mechanics. Archives of Computational Methods in Engineering, 2(1):49-138, mar 1995.

[4] F. Auricchio, P. Carotenuto, and A. Reali. On the geometrically exact beam model: A consistent, effective and simple derivation from three-dimensional finite-elasticity. International Journal of Solids and Structures, 45(17):47664781, aug 2008.

[5] K. Bathe and S. Bolourchi. Large displacement analysis of three-dimensional beam structures. International Journal for Numerical Methods in Engineering, 14(7):961-986, jan 1979.

[6] J. Battini. Large Rotations and Nodal Moments in Corotational Elements. CMES, 33(1):1-15, 2008.

[7] J. Battini and C. Pacoste. Co-rotational beam elements with warping effects in instability problems. Computer Methods in Applied Mechanics and Engineering, 191(17-18):1755-1789, feb 2002.

[8] P. Betsch and P. Steinmann. Frame-indifferent beam finite elements based upon the geometrically exact beam theory. International Journal for Numerical Methods in Engineering, 54(12):1775-1788, aug 2002.

[9] P. Betsch and P. Steinmann. Constrained dynamics of geometrically exact beams. Computational Mechanics, 31(1-2 SPEC.):49-59, may 2003. 
[10] R. Blevins. Flow - induced vibration. Krieger Publishing Company, 2 edition, 1994.

[11] J. Bonet and R. D. Wood. Nonlinear Finite Element Methods. Springer Berlin Heidelberg, Berlin, Heidelberg, 2008.

[12] A. Cardona and M. Geradin. A beam finite element nonlinear theory with finite rotations. International Journal for Numerical Methods in Engineering, 26(11):2403-2438, nov 1988.

[13] A. Cardona and M. Geradin. A beam finite element nonlinear theory with finite rotations. International Journal for Numerical Methods in Engineering, 26(11):2403-2438, nov 1988.

[14] P. Češarek, M. Saje, and D. Zupan. Kinematically exact curved and twisted strain-based beam. International Journal of Solids and Structures, 49(13):18021817 , jun 2012.

[15] R. Chandrupatla, D. Belegundu, T. Ramesh, and C. Ray. Introduction to finite elements in engineering. Pearson Education, 1997.

[16] A K Chopra. Dynamics of Structures: International Edition. Pearson Education Limited, 4 edition, 2015.

[17] M. A. Crisfield. A consistent co-rotational formulation for non-linear, threedimensional, beam-elements. Computer Methods in Applied Mechanics and Engineering, 81(2):131-150, aug 1990.

[18] M. A. Crisfield, U. Galvanetto, and G. Jelenić. Dynamics of 3-D co-rotational beams. Computational Mechanics, 20(6):507-519, nov 1997.

[19] M. A. Crisfield, G. F. Moita, L. P. R. Lyons, and G. Jelenić. Enhanced lowerorder element formulations for large strains. Computational Mechanics, 17(12):62-73, dec 1995.

[20] M.A Crisfield. Non-linear Finite Element Analysis of Solids and Structures Volume 2, volume 1. Wiley, 1991.

[21] J. S Dai. Euler-Rodrigues formula variations, quaternion conjugation and intrinsic connections. Mechanism and Machine Theory, 92:144-152, 2015.

[22] H A Elkaranshawy and M A Dokainish. Corotational finite element analysis of planar flexible multibody systems. Computers \& Structures, 54(5):881-890, mar 1995. 
[23] L Euler. Additamentum I de curvis elasticis. Metodus inveniendi lieas maximi minimive proprietate gaudentes. Lausanne., 1744.

[24] M. Geradin and A. Cardona. Kinematics and dynamics of rigid and flexible mechanisms using finite elements and quaternion algebra. Computational Mechanics, 4(2):115-135, 1988.

[25] F. Gruttmann, R. Sauer, and W. Wagner. A geometrical nonlinear eccentric 3D-beam element with arbitrary cross-sections. Computer Methods in Applied Mechanics and Engineering, 160(3-4):383-400, jul 1998.

[26] K. M. Hsiao. Corotational total Lagrangian formulation for three-dimensional beam element. AIAA Journal, 30(3):797-804, mar 1992.

[27] K. M. Hsiao, J. Y. Lin, and W. Y. Lin. A consistent co-rotational finite element formulation for geometrically nonlinear dynamic analysis of 3-D beams. Computer Methods in Applied Mechanics and Engineering, 169(1-2):1-18, jan 1999.

[28] A. Ibrahimbegović. On finite element implementation of geometrically nonlinear Reissner's beam theory: three-dimensional curved beam elements. Computer Methods in Applied Mechanics and Engineering, 122(1-2):11-26, apr 1995.

[29] A. Ibrahimbegovic. On the choice of finite rotation parameters. Computer Methods in Applied Mechanics and Engineering, 149(1-4):49-71, oct 1997.

[30] A. Ibrahimbegovic. On the choice of finite rotation parameters. Computer Methods in Applied Mechanics and Engineering, 149(1-4):49-71, oct 1997.

[31] A Ibrahimbegović, F. Frey, and I Kožar. Computational aspects of vector-like parameterization of three-dimensional finite rotations. International Journal for Numerical Methods in Engineering, 38(21):3653-3673, 1995.

[32] A. Ibrahimbegović and M. Mikdad. Finite rotations in dynamics of beams and implicit timestepping schemes. International Journal for Numerical Methods in Engineering, 41(November 1996):781-814, mar 1998.

[33] M. Iura and S. N. Atluri. Dynamic analysis of finitely stretched and rotated three-dimensional space-curved beams. Computers and Structures, 29(5):875889, 1988. 
[34] M. Iura and S. N. Atluri. On a consistent theory, and variational formulation of finitely stretched and rotated 3-D space-curved beams. Computational Mechanics, 4(2):73-88, 1988.

[35] G Jelenic and M. A. Crisfield. Interpolation of Rotational Variables in Nonlinear Dynamics of 3D Beams. International Journal for Numerical Methods in Engineering, 1222(February 1997):1193-1222, dec 1998.

[36] G. Jelenić and M.A. Crisfield. Geometrically exact 3D beam theory: implementation of a strain-invariant finite element for statics and dynamics. Computer Methods in Applied Mechanics and Engineering, 171(1-2):141-171, mar 1999.

[37] G. Jelenić and M. Saje. A kinematically exact space finite strain beam model - finite element formulation by generalized virtual work principle. Computer Methods in Applied Mechanics and Engineering, 120(1-2):131-161, jan 1995.

[38] T. N. Le, J. M. Battini, and M. Hjiaj. Efficient formulation for dynamics of corotational 2D beams. Computational Mechanics, 48(2):153-161, 2011.

[39] T. N. Le, J. M. Battini, and M. Hjiaj. Dynamics of 3D beam elements in a corotational context: A comparative study of established and new formulations. Finite Elements in Analysis and Design, 61:97-111, nov 2012.

[40] T. N. Le, J. M. Battini, and M. Hjiaj. A consistent 3D corotational beam element for nonlinear dynamic analysis of flexible structures. Computer Methods in Applied Mechanics and Engineering, 269:538-565, feb 2014.

[41] Z. X. Li. A co-rotational formulation for 3D beam element using vectorial rotational variables. Computational Mechanics, 39(3):309-322, nov 2007.

[42] J. M. Mäkinen. Total Lagrangian Reissner's geometrically exact beam element without singularities. International Journal for Numerical Methods in Engineering, 70(9):1009-1048, may 2007.

[43] J. M. Mäkinen. Total Lagrangian Reissner's geometrically exact beam element without singularities. International Journal for Numerical Methods in Engineering, 70(9):1009-1048, may 2007.

[44] C. Oran and A. Kassimali. Large deformations of framed structures under static and dynamic loads. Computers \& Structures, 6(6):539-547, dec 1976.

[45] M. P. Paidoussis. Fluid-structure interactions. Volume 1, Slender structures and axial flow. Academic Press, 1998. 
[46] E. Petrov and M. Géradin. Finite element theory for curved and twisted beams based on exact solutions for three-dimensional solids Part 2: Anisotropic and advanced beam models. Computer Methods in Applied Mechanics and Engineering, 165(1-4):93-127, nov 1998.

[47] C. C. Rankin and F. A. Brogan. An element independent corotational procedure for the treatment of large rotations. Journal of pressure vessel technology, 108(2):165-174, may 1986.

[48] S. Rao. Vibration of Continuous Systems. John Wiley \& Sons, 2007.

[49] E. Reissner. On one dimensional finite strain Beam theory: the plane problem. Journal of Applied Mathematics and Physics, 23:795-804, 1972.

[50] E. Reissner. On one-dimensional finite-strain beam theory: The plane problem. ZAMP Zeitschrift für angewandte Mathematik und Physik, 23(5):795-804, sep 1972.

[51] E. Reissner. On finite deformations of space-curved beams. ZAMP Zeitschrift für angewandte Mathematik und Physik, 32(6):734-744, 1981.

[52] J. C. Simo, N. Tarnow, and M. Doblare. Non-linear dynamics of threedimensional rods: Exact energy and momentum conserving algorithms. International Journal for Numerical Methods in Engineering, 38(9):1431-1473, may 1995.

[53] J. C. Simo and L. Vu-Quoc. A three-dimensional finite-strain rod model. part II: Computational aspects. Computer Methods in Applied Mechanics and Engineering, 58(1):79-116, oct 1986 .

[54] J. C. Simo and L. Vu-Quoc. On the Dynamics of Flexible Beams Under Large Overall MotionsThe Plane Case: Part I. Journal of Applied Mechanics, 53(86):849-854, dec 1986.

[55] J. C. Simo and L. Vu-Quoc. On the Dynamics of Flexible Beams Under Large Overall MotionsThe Plane Case: Part I. Journal of Applied Mechanics, 53(86):849-854, dec 1986.

[56] J. C. Simo and L. Vu-Quoc. On the dynamics in space of rods undergoing large motions - A geometrically exact approach. Computer Methods in Applied Mechanics and Engineering, 66(2):125-161, feb 1988. 
[57] M. W. Smolenski. Statically and kinematically exact nonlinear theory of rods and its numerical verification. Computer Methods in Applied Mechanics and Engineering, 178:89-113, 1999.

[58] B. Sreejith, K. Jayaraj, N. Ganesan, C. Padmanabhan, P. Chellapandi, and P. Selvaraj. Finite element analysis of fluid-structure interaction in pipeline systems. Nuclear Engineering and Design, 227(3):313-322, 2004.

[59] J. Stuelpnagel. On the Parametrization of the Three-Dimensional Rotation Group. SIAM Review, 6(4):422-430, 1964.

[60] H. B. Wen, Y. R. Yang, P. Li, Y. D. Li, and Y. Huang. A New Method Based on Laplace Transform and Its Application to Stability of Pipe Conveying Fluid. Shock and Vibration, 2017:1-9, 2017.

[61] P. Wriggers. Nonlinear Finite ELement Methods, volume 1. Springer, 2008.

[62] OC Zienkiewicz and RL Taylor. The finite element method for solid and structural mechanics. Elsevier Butterworth-Heinemann, 2005.

[63] E. Zupan, M. Saje, and D. Zupan. Dynamics of spatial beams in quaternion description based on the Newmark integration scheme. Computational Mechanics, 51(1):47-64, apr 2012. 